\title{
Kinematic Modelling of a Panel ENCLOSED MECHANISM
}

\author{
By \\ Aaron Yu, B.Eng \\ Aerospace Engineering \\ Ryerson University, 2012
}

A thesis presented to Ryerson University

in partial fulfillment of the
requirements for the degree of

Master of Applied Science

in the program of

Aerospace Engineering

Toronto, Ontario, Canada, 2015

CAaron Yu 2015 


\section{AUTHOR's DeClaration}

I hereby declare that I am the sole author of this thesis. This is a true copy of the thesis, including any required final revisions, as accepted by my examiners.

I authorize Ryerson University to lend this thesis to other institutions or individuals for the purpose of scholarly research.

I further authorize Ryerson University to reproduce this thesis by photocopying or by other means, in total or in part, at the request of other institutions or individuals for the purpose of scholarly research.

I understand that my thesis may be made electronically available to the public. 


\title{
AbSTRACT
}

\section{Kinematic Modelling of a Panel Enclosed Mechanism}

\author{
Aaron $\mathrm{Yu}$ \\ Master of Applied Science, 2015 \\ Department of Aerospace Engineering, Ryerson University
}

This thesis presents a new method for kinematic modeling and analysis of a six degree-offreedom parallel robot enclosed by a number of sliding panels, called panel enclosed mechanism. This type of robots has been seen in applications where mechanisms are covered by changeable surfaces, such as aircraft morphing wings made of variable geometry truss manipulators. Based on the traditional parallel robot kinematics, the proposed method is developed to model the motions of a multiple segmented telescopic rigid panels that are attached to the moving branches of the mechanism. Through this modeling and analysis, a collision detection algorithm is proposed to analyze the collisions that could occur between adjacent sliding panels during motion over the workspace of the mechanism. This algorithm will help to design a set of permissible panels used to enclose the mechanism free of collision. A number of cases are simulated to show the effectiveness of the proposed method. In addition, an extra link is added to provide an additional degree-of-freedom. Various search methods are employed to evaluate optimal orientation angles to minimize collisions of adjacent panels. Finally, the effect of increased mobility is analyzed and validated as a potential solution to reduce panel collisions. 


\section{ACKNOWLEDGEMENTS}

I would like to acknowledge the staff and professors of Ryerson University for the opportunity and knowledge to achieve the skills necessary as an aerospace engineer. I wish to sincerely thank my thesis supervisor, Dr. Jeff Xi, for the providing knowledge, guidance, and support needed to complete this thesis. I look forward to working with him for future Ph.D. studies.

I wish to thank Dr. Daniel Finistauri for the advice and getting me started with this thesis. Your works and opinions were much appreciated for the completion of this thesis.

Finally, I would like to sincerely thank my family and close friends for their encouragement, support, and understanding over these years. 


\section{TABLE OF CONTENTS}

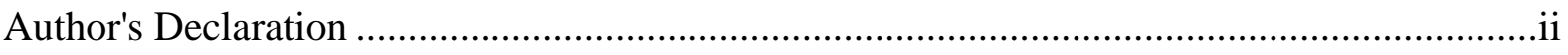

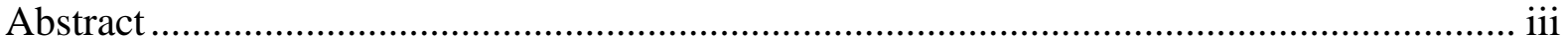

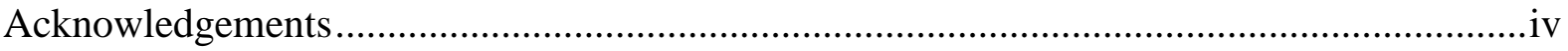

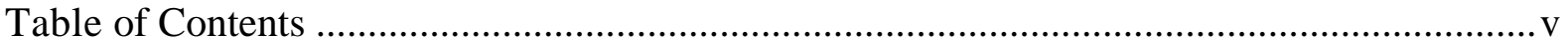

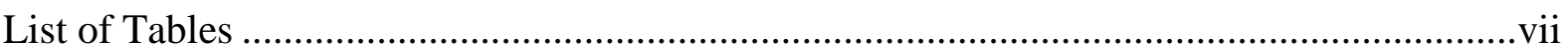

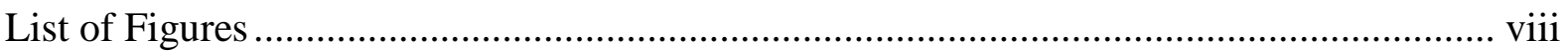

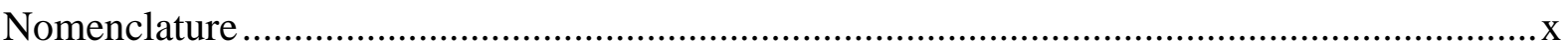

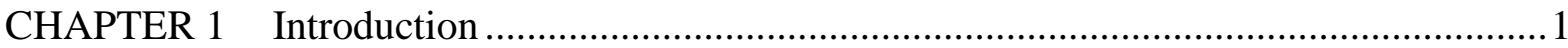

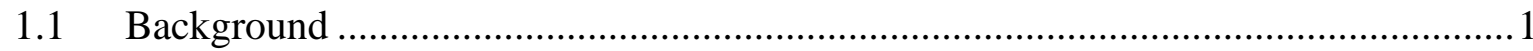

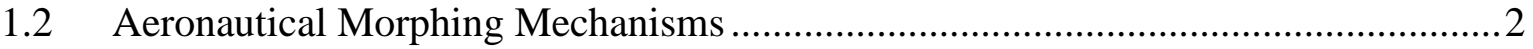

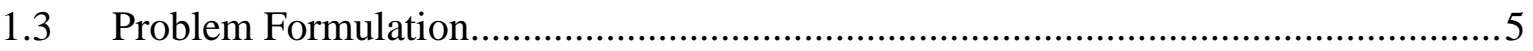

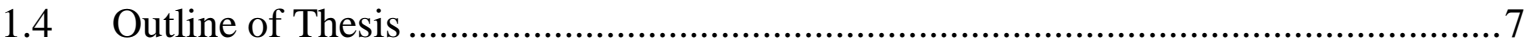

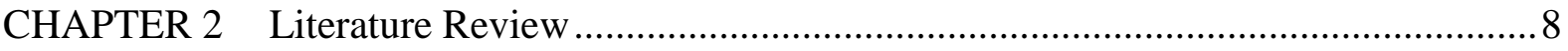

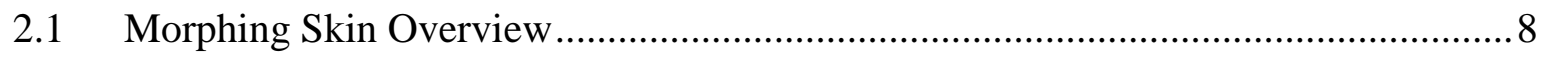

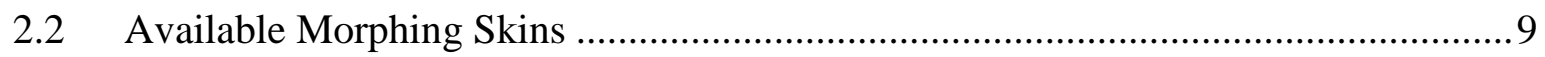

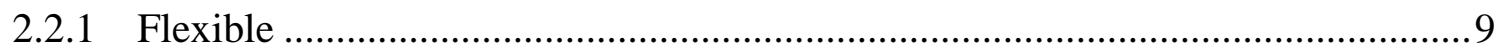

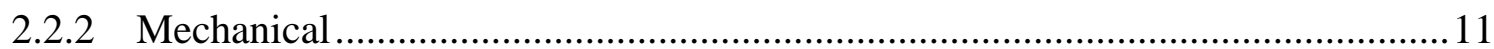

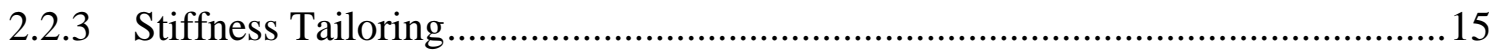

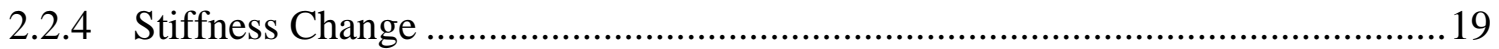

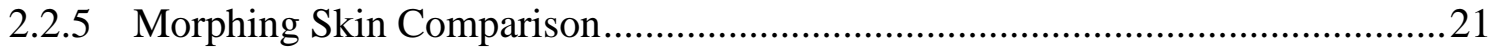

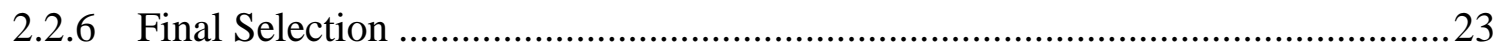

CHAPTER 3 Kinematic Modeling of An Active Panel ....................................................25

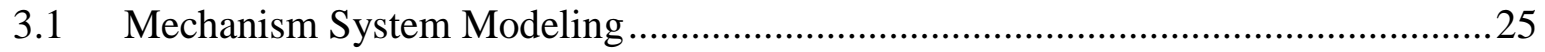

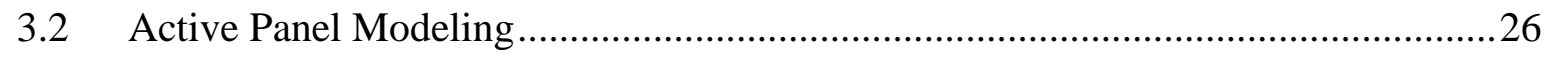

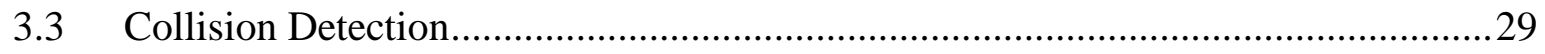

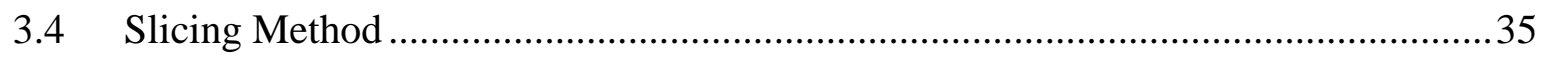




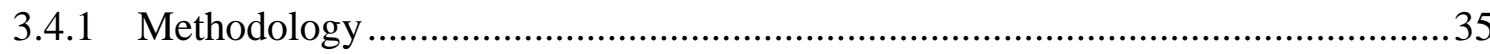

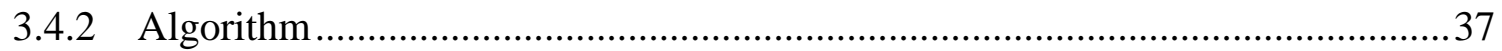

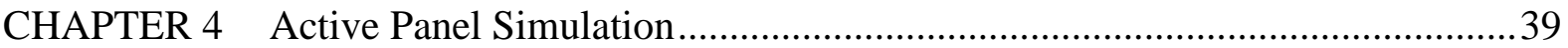

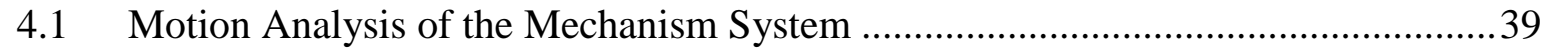

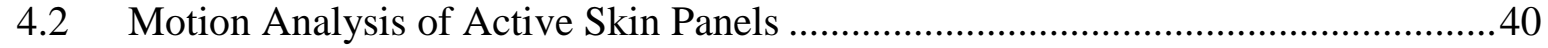

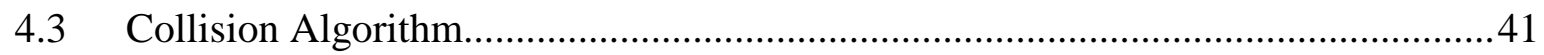

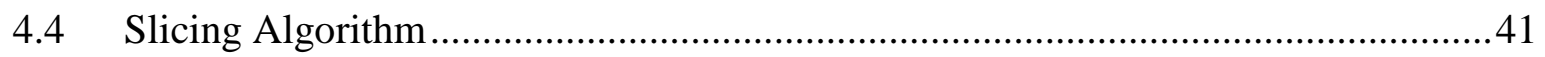

CHAPTER 5 Kinematic Modelling of A Passive Panel .....................................................44

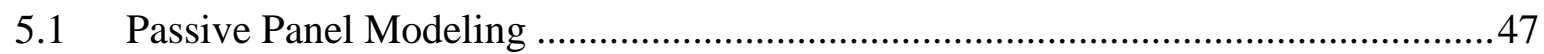

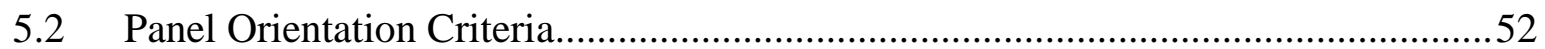

5.3 Linear Formulation: Newton-Raphson for Panel Orientation Optimization.............57

5.4 Non-Linear Formulation: Multiobjective Optimization for Panel Orientation

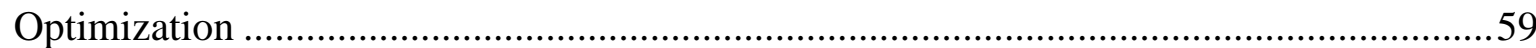

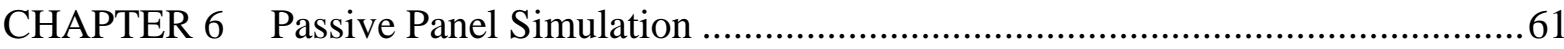

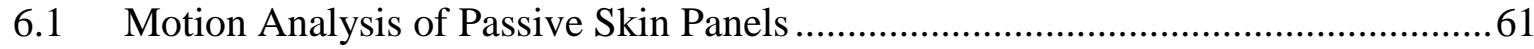

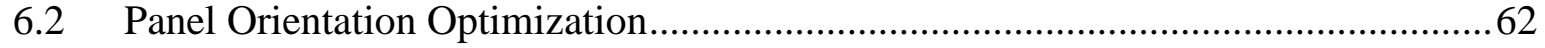

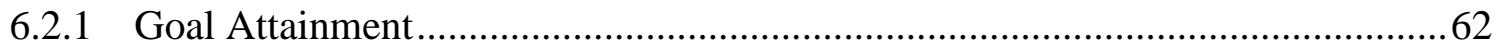

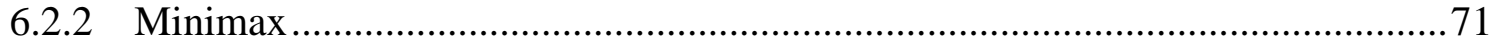

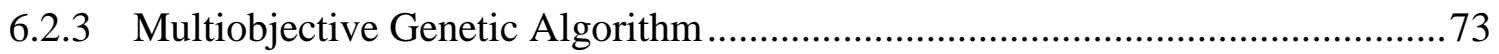

6.3 Methodology Application to Morphing Skin .................................................... 77

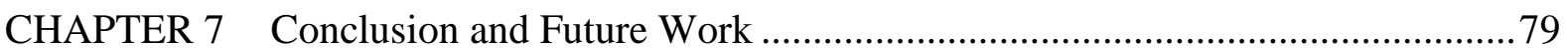

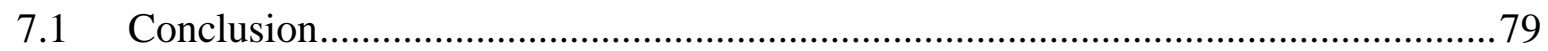

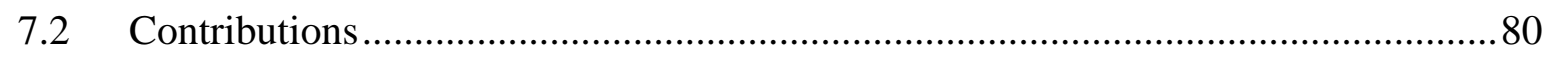

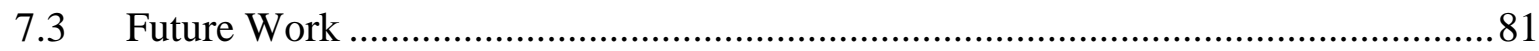

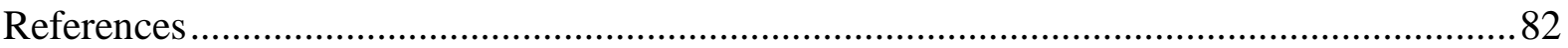




\section{LIST OF TABLES}

Table 1: Baseline orientation criteria results 62

Table 2: Goal attainment criteria results - baseline orientation .............................................63

Table 3: Goal attainment criteria results - 10[deg] global z-axial twist ...............................66

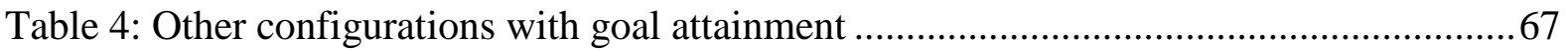

Table 5: Minimax criteria results - 10[deg] global z-axial twist ......................................... 73

Table 6: Multiobjective GA criteria results - 10[deg] global z-axial twist.............................76

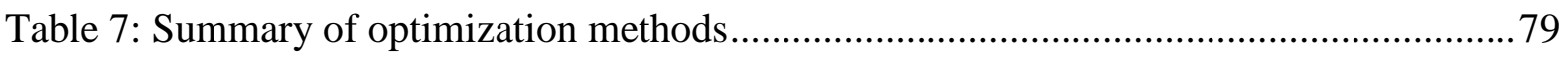




\section{LIST OF FIGURES}

Fig. 1: (a) and (b): Morphing winglet front view and side view; (c): morphing winglet module with sliding panels, all adopted from [6] 1

Fig. 2 Ryerson University VGTM morphing mechanism [6] ................................................4

Fig. 3: (a) Positive Poisson's ratio (b) auxetic material, all adopted from [32] ....................... 10

Fig. 4: (a) strand with a central ellipse (b) strand with central hexagon (c) strand with central

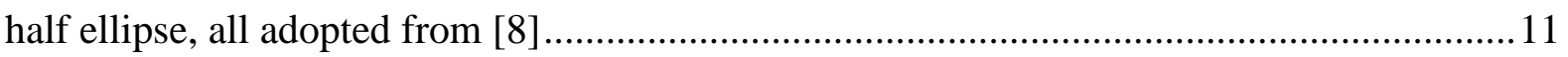

Fig. 5: Sample of different origami patterns adopted from [38] ....................................... 12

Fig. 6: (a) collapsed flat-folded structure (b) fully deployed structure, all adopted from [39] 13

Fig. 7: Schematic kirigami structure for a single module adopted from [41] ........................ 14

Fig. 8: Custom testing apparatus for fiber matrix composite adopted from [43] .................. 16

Fig. 9: Hydrofoil under deflection adopted from [47] ........................................................ 18

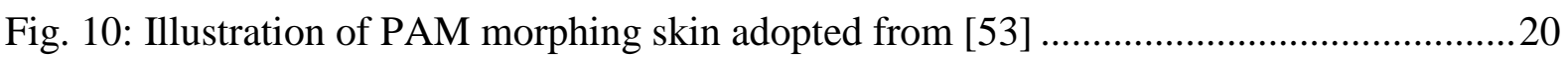

Fig. 11: (a) Sliding panel prototype leading edge view (b) Top view, all adopted from [6] ...23 Fig. 12: (a) Definition of hexapod configuration, (b) Definition of skin panel configuration 28

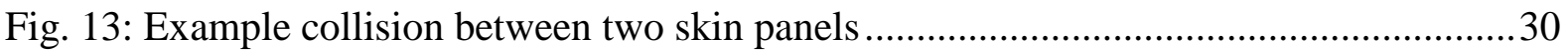

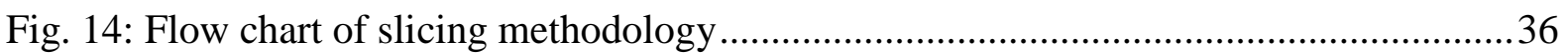

Fig. 15: Example of slicing algorithm. Left panel has two sets of collisions. Once sliced, the

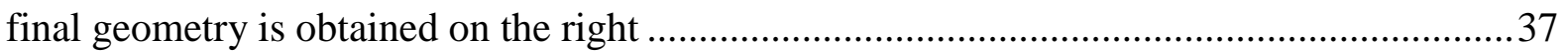

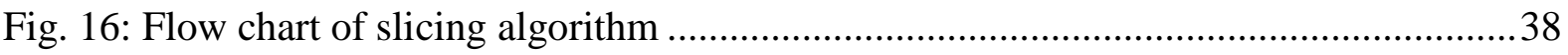

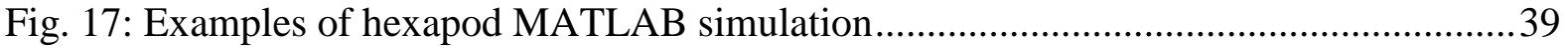

Fig. 18: Example of hexapod with skin panels. The orientation is a 10 [deg] twist around the global z-axis

Fig. 19: Example of program output for collision algorithm. This figure shows all collision points on each individual panel. 
Fig. 20: Slicing algorithm result for one panel. Three different orientations have been tested on this panel and the final shape is shaded

Fig. 21: Close-up of slicing.....

Fig. 22: Hexapod with sliced active panels

Fig. 23: Extended link of sliding panels adopted from [64] 44

Fig. 24: Schematic of sliding panel prototype .45

Fig. 25: Schematic of extra link for analysis .46

Fig. 26: Definition of passive panel configuration for base (a) and platform (b) .48

Fig. 27: Example of hexapod with passive skin panels. Baseline orientation 61

Fig. 28: Goal attainment for baseline orientation 63

Fig. 29: Passive panel orientation with goal attainment for 10 [deg] global z-axial twists. (a) Isometric view (b) top view .65

Fig. 30: Goal attainment for 10[deg] global z-axial twist .65

Fig. 31: Passive panel orientation with Minimax for baseline orientation .71

Fig. 32 Passive panel orientation with Minimax for 10[deg] global z-axial twist 72

Fig. 33: Minimax for 10[deg] global z-axial twist .72

Fig. 34: Passive panel orientation with multiobjective GA for baseline orientation .74

Fig. 35: Passive panel orientation with multiobjective GA for 10[deg] global z-axial twist .. 76 


\section{NOMENCLATURE}

\begin{tabular}{|c|c|}
\hline Symbol & Definition \\
\hline$x, y, z$ & $\mathrm{x}, \mathrm{y}$, or $\mathrm{z}$ co-ordinate position \\
\hline$O$ & fixed co-ordinate frames \\
\hline $\boldsymbol{b}$ & base vectors \\
\hline $\boldsymbol{r}$ & mechanism branch vectors \\
\hline $\boldsymbol{h}$ & Platform positional vector \\
\hline$p$ & Platform vectors \\
\hline $\boldsymbol{R}$ & Rotational matrix \\
\hline $\boldsymbol{\theta}$ & Rotation angles \\
\hline$s$ & Panel point vectors \\
\hline$s^{\prime}$ & Local panel point vectors \\
\hline$n$ & Normal vector between the base and branch \\
\hline$l$ & SSI intersection line function \\
\hline$t$ & Function parameterization variable \\
\hline$g$ & Panel edge function \\
\hline$c$ & Base extra link \\
\hline$d$ & Platform extra link \\
\hline$q$ & Position vectors \\
\hline Subscript & Definition \\
\hline$i$ & $i$-th mechanism branch \\
\hline int & intersection \\
\hline$j$ & $j$-th point vector \\
\hline$b$ & base \\
\hline$p$ & platform \\
\hline$s$ & panel \\
\hline$S$ & S-joint \\
\hline$R$ & R-joint \\
\hline
\end{tabular}

Throughout the thesis, bold lower case indicates vector; bold upper case indicates matrix; and regular lower or upper case indicates scalar 


\section{CHAPTER 1 INTRODUCTION}

\subsection{BACKGROUND}

Over the last four or five decades, parallel mechanisms/robots have been developed from the initial application for flight simulators to a wide range of other applications including parallel kinematic machines (PKM)[1], six-axis force sensors[2], haptic devices[3], telescope mirror platforms[4], and so on. However, these past applications only required research and development of the mechanism structure. Recently, there is a new development on mechanisms covered by changeable surfaces, such as aircraft morphing wings developed based on the principle of variable geometry truss manipulators (VGTM) [5], which is made of a series of parallel mechanism modules. Fig. 1 (a) and (b) shows a morphing winglet developed by Ryerson University using two parallel mechanism modules. Fig. 1 (c) shows a morphing winglet module covered with a morphing skin made of a set of sliding panels.

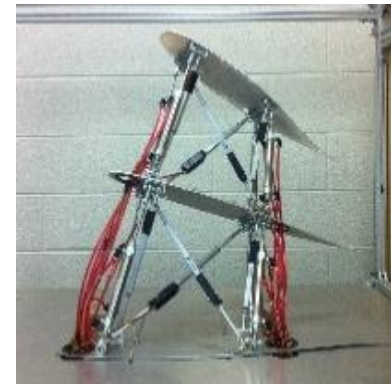

(a)

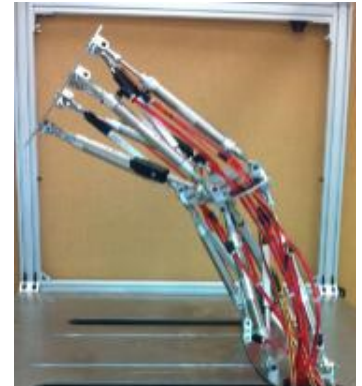

(b)

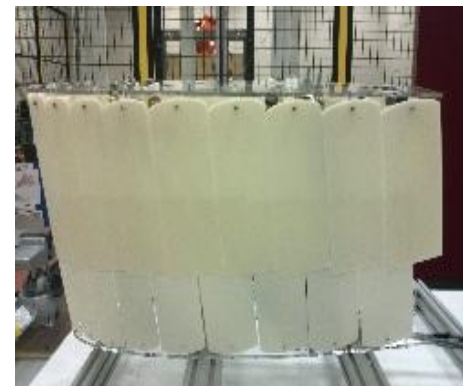

(c)

Fig. 1: (a) and (b): Morphing winglet front view and side view; (c): morphing winglet module with sliding panels, all adopted from [6]

In addition to aircraft morphing wings, other applications may require parallel robots to be covered due to environment requirement, for example, medical robots, industrial robots, and nuclear robots. These coverings can be classified as flexible and rigid. 
Flexible covers are stretchable materials [7,8]. Though easy to make, there are a number of problems, such as sagging, ripples, and additional actuation forces for material stretching $[9,10]$. Rigid covers are made of a series of rigid sliding panels, no sagging and no ripple with negligible actuation force, this is good for applications where structural strength is required, such as morphing winglet[6]. This background motivates the research reported here.

In this thesis, we call a parallel robot covered with sliding panels a panel enclosed mechanism. This problem calls for the development of a new method for kinematic modeling and analysis of this type of parallel robots. The method developed here models the motions of a set of rigid panels, which are attached to the moving branches of a morphing mechanism. Through modeling and analysis, a collision detection (CD) algorithm is used to analyze the collisions that could occur between adjacent sliding panels during motion over the workspace of the enclosed mechanism. This algorithm can help to design a set of permissible panels used to enclose the mechanism free of collision.

\subsection{Aeronautical Morphing Mechanisms}

Biomimetics, known as the study of biological systems as solutions for design and engineering, is also the origin for the term morphing. Biomimetics aim to replicate nature's mechanics to achieve optimal functionality with current engineering designs [11]. In the case of the morphing wing, the intention was to manufacture a wing that is capable of seamless transitions similar to a bird's wing. However, such feats are often complex due to the fact that the transitions are dependent on environmental factors, accuracy of sensory hardware, and responsiveness of the morphing mechanism. Therefore morphing wings are designed to adapt to the flight mission profile, 
resulting in morphing wings being both a biomimicry design and also an adaptive structure [12].

A general flight mission profile includes taxiing, take-off, cruising, and landing. Most aircrafts are designed specifically for optimal cruising, therefore resulting in lower camber airfoils that sacrifices the ground roll distance, take-off, and landing performance. To compensate for different flight mission profiles, wing morphing is essential. The most common and conventional forms of single degree-of-freedom (DOF) morphing include airfoil[13-15], twist[16-18], sweep [19, 20], span[21, 22], and cant morphing[23, 24].

All the aforementioned morphing is limited to a single DOF. In other words, the aircraft is limited to a single type of morphing. This realization led to the introduction of poly morphing. As the name implies, it is an ambitious approach to blend the benefits of each individual single DOF morphing into one single design. The goal is to achieve a wing design that is capable of optimal function in nearly every regime of the flight mission profile. To facilitate such an idea requires an extremely complex structure. The structure must be capable of providing sufficient kinematic motions as well as structural integrity. Numerous associations and institutes have tackled this problem and developed the some of the following: Air Force Research Laboratory (AFRL) Z-wing [25], NextGen MFX-1 wing design [25, 26], smart memory alloy (SMA) wing designs [27-29], and Ryerson University VGTM morphing wing [6].

For a minimum three DOF poly morphing, the mechanism design must possess robust structural integrity, versatility, and support complex kinematics. Therefore, Ryerson 
University proposed the use of truss-based parallel robot mechanism, VGTM, as a structural design for morphing wings (Fig. 2).

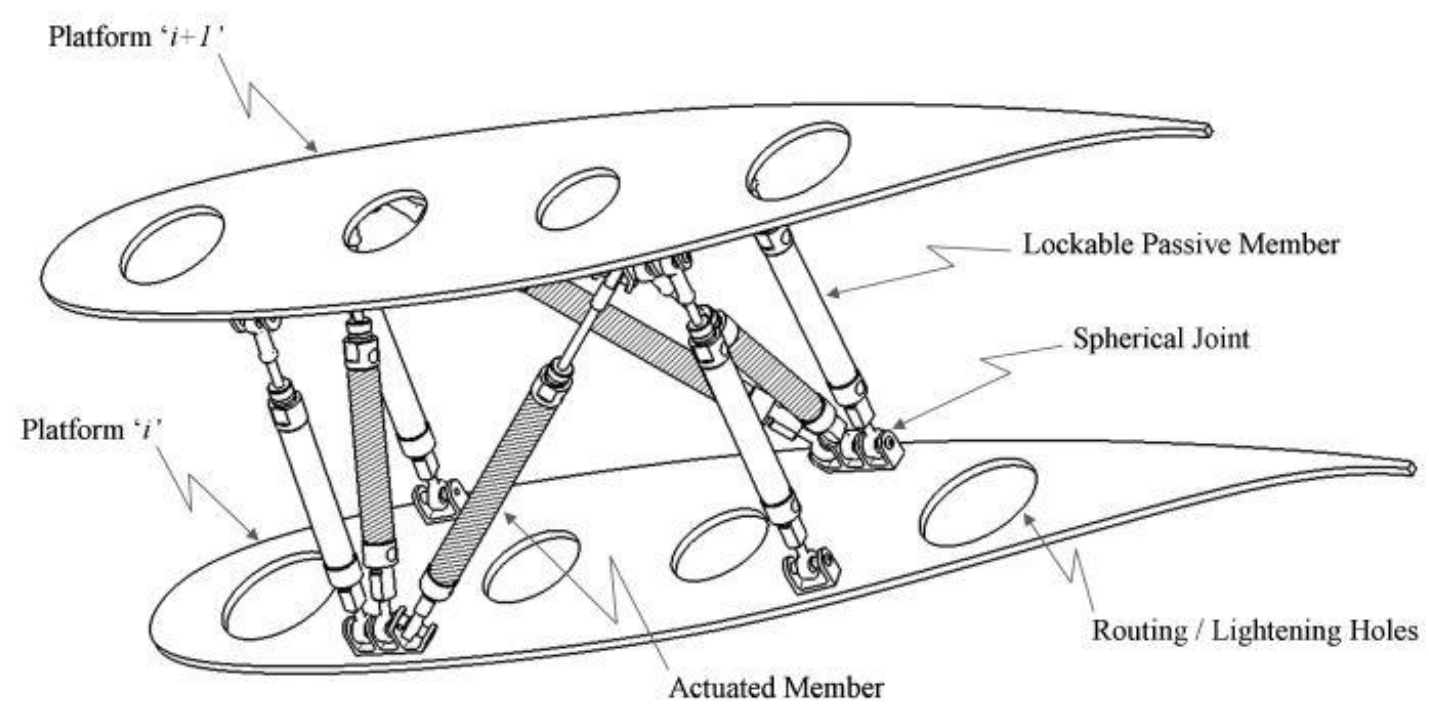

Fig. 2 Ryerson University VGTM morphing mechanism [6]

The developed morphing wing is designed around the basis of modularizing an entire wing into individual parallel robots. Where the combination of VGTM and modularity provides versatility, and the VGTM alone provide the kinematics and structural integrity. In the case of a wing design, the end-effector of a single module VGTM would be an airfoil cross section. Additional VGTM modules may be added on to the preceding end-effector to create a series of parallel robots. A serial configuration may enhance the capabilities of seamless morphing, streamlining the morphed geometry [6]. Each module of the morphing wing is not susceptible to conventional actuation control. This is due to its kinematically indeterminate property, all truss members may not be actuated at the same time without sacrificing structural rigidity. Therefore, an underactuation method is required to replace conventional control. Underactuation is possible to support passive members within the VGTM system. This helps maintain torsional and structural stiffness $[5,6,30,31]$. 


\subsection{Problem Formulation}

A morphing mechanism has been developed and tested in Ryerson University. However, to this date, still no definitive morphing skin structure is fully compatible with such versatile structure. Fortunately, there is a potential morphing skin design that utilizes rigid skin panels. Similar to the morphing wing modularity concept, the telescopic rigid skin panels are modularized span-wise. The only existing documentations regarding this design are only working prototypes with no numerical formulations or experimental results. Therefore, this thesis is to provide a numerical validation of said design.

An ideal morphing skin design will be able to compensate for any degrees of motion. Intuitively, the ideal skin will composed of flexible material, such as synthetic fibers. However, such designs usually provide miniscule protection to external forces. Therefore, if a certain level of protection is required, materials with higher stiffness are favoured. Although a higher stiffness may improve resistance against external forces, the drawback is the restriction to the robots allowable motion and the generation of surface deformities during morphing. This dilemma demands a morphing skin design that is versatile and structurally supportive.

The proposed design in this thesis aims to enclose a six DOF robot with a rigid morphing skin. A hexapod was selected for the nature of its simplicity and symmetry as a six DOF robot. It is assumed that the platform and base of the hexapod are geometrically similar. This is to provide a simpler representation of the simulation. If required, the algorithms presented are applicable to geometrically indifferent platforms and bases. Naturally, a single rigid skin panel is incapable of adapting to any morphing motion. However, when segmented into individual panels span-wise 
and given telescopic properties, it may compensate for axial translational of the robot. Given that, each panel is intentionally aligned to be flush with its adjacent panels, it will restrict any sort of axial rotation. From this, an obvious issue of surface-tosurface intersection is present. Two solutions were suggested to resolve this: active panel solution and passive panel solution.

The active panel configuration assumes the skin panels to be rigidly attached to the members of the robot. By doing so, the motion of the panel is directly determined by the orientation of each member of the robot. The active panel methodology views potential collisions generated by the robot's workspace as an adverse anomaly and removes it. Overall, this method aims to optimize the shape of the skin panel with respect to the robot's designed workspace. With the removal of material, voids will be present in the overall design. To fill these newly formed gaps, flexible materials will be used or a second layer of rigid material behind the gaps for a stiffer alternative. As mentioned, large reliability on using flexible materials will result in a loss of stiffness and increase in surface deformation. However, if the usage of flexible material is minimized, it can provide versatility to a rigid panel design. The passive panel aims to add an extra link between the robot and the panel, attached between a revolute joint (R-joint) and a spherical joint (S-joint) respectively. This configuration is to avoid removal of material from each panel and to allow panels to orientate into an ideal location based on the interactions among adjacent panels. Overall, this alternate method optimizes the skin panel orientation based on the robot's designed workspace. This thesis will outline the numerical formulation of the mechanism along with the skin panels. Both active and passive skin panel methodology will be validated with MATLAB simulations. 


\subsection{OUTLINE OF THESIS}

The remaining thesis is organized as follows:

Chapter 2 provides a literature review of existing morphing skin designs, focusing on flexible, mechanical, and stiffness tailored skin designs.

Chapter 3 presents a kinematic modeling method for the active skin panel and the robot mechanism. Collision detection method will also be introduced along with a prognostic solution to active panel problem.

Chapter 4 discusses the motion analysis of the robotic mechanism and the active skin panel, along with the methodology validation.

Chapter 5 presents a kinematic modeling method for the passive skin panel. Linear (Newton-Raphson) and non-linear formulations will be experimented to optimize panel orientation.

Chapter 6 discusses the motion analysis of the passive skin panel, along with the methodology validation.

Chapter 7 provides concluding remarks of this thesis along with future work. 


\section{CHAPTER 2 LITERATURE REVIEW}

In this chapter, existing morphing skin designs will be presented and discussed. The proposed morphing skin design by Ryerson University will be presented and numerically simplified for the ease of kinematic modeling.

\subsection{MORPHING SKIN OVERVIEW}

From an aircraft design standpoint, an ideal morphing skin for a lifting surface should maintain a given airfoil geometry, while providing a smooth and streamline profile during and after a morph. In addition, the skin is required to support the structural integrity of the overall lifting surface, ultimately reducing material and weight of the overall wing design. Conventional aircraft skins are capable of satisfying all of the above requirements because they are unable to change shape over the course of its operation. With the addition of morphing, the skin design must be versatile, which will usually result in the loss of stiffness (use of flexible materials) or increase in structural complexity (use of rigid morphing mechanisms). With the lack of stiffness, reinforcement of the internal structure is necessary to compensate for this loss. However, if a skin design is too flexible, like synthetic fibers with high elasticity, the skin will be incapable of maintaining its geometry without introducing wrinkles/ripples and other possible deformities on to lifting surface. In addition, external loading will deform flexible materials, which will in turn alter the geometry of the overall wing structure. This causes an aerodynamically unstable lifting that will hinder the overall performance of the aircraft. Therefore, an ideal aircraft morphing skin design is required to be versatile, structurally supportive, and aerodynamically viable. 


\subsection{Available Morphing SKInS}

Due to the recent emergence of morphing skin studies, there are currently only a handful of documentations regarding this technology. Most of these studies share similar design traits and will therefore be grouped together based on their morphing structure and skin combinations.

\subsubsection{FLeXIBLE}

There is still a need for a material that provides both a flexibility and load bearing capability. However to achieve high flexibility, the ability to bear load must be sacrificed and vice versa. Despite this, a few materials and methods have been developed to compensate the dilemma between flexibility and load bearing capability. They include elastomers, auxetic materials, and flexible skin enclosed cellular structure.

\section{ELASTOMERS}

Elastomers also known as rubber is a very common flexible skin material for morphing wing designs. DARPA smart wing program uses high strain-to-failure silicone flexible skin material. However due to its flexible nature, additional mechanisms are required to recover original shape upon unloading of skin. Given that elastomers have non-linear stress strain relationships and sensitivity to glass transition temperatures, elastic modulus will vary with strain and the transition between a rubber and glass properties will vary with temperature. Such properties make it difficult to bear loads. In addition, operation periods of the elastomers may be a concern due to variable strain relationships, hence leading to issues of fatigue $[19,20,32]$. 


\section{AuXetic Materials}

Auxetic materials are materials that carry a negative Poisson's ratio. Contrary to materials with positive Poisson's ratio, when the material is compressed, it will become narrower. When stretched, it will become wider (Fig. 3). Auxetic materials include foams, ceramics, composites, and polymers. Negative Poisson's ratio is achieved on a micro-scale, the material generally possess a re-entrant cell-like structure. Although potentially viable for morphing structures, auxetic material was deemed more useful as a filler for voids and possible gaps on an aerodynamic surface, for example to provide a seamless transition between wing an aileron [32].

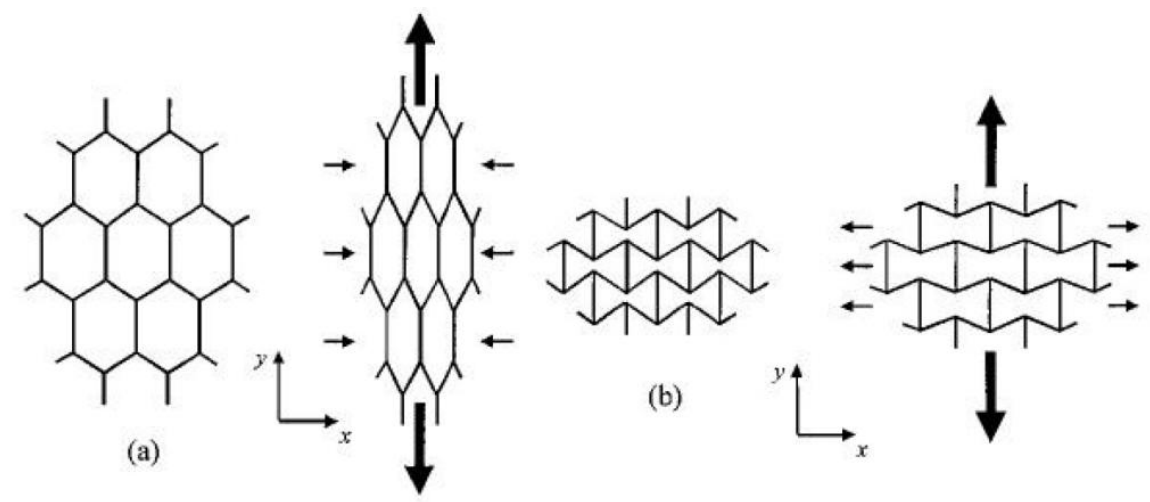

Fig. 3: (a) Positive Poisson's ratio (b) auxetic material, all adopted from [32]

\section{SUPPORTED FLEXIBLE SKINS}

A popular alternative or using a fully flexible skin while retaining sufficient structural stiffness to bear loads, is through the use of a cellular structure (ex. Honeycomb [7, 9, 33, 34], semi-elliptical [10, 35], and uniquely-shaped cells $[8,36])$ covered by a flexible skin, which is generally a flexible matrix composite. The uniquely-shaped cells (Fig. 4), notably the one developed by Olympio et al [8], their design consists of cellular substructures within a primary cell, which applies pretension to reduce wrinkle formation of the flexible skin and provides strain relief. Wrinkle or ripple formation is a common inevitable problem in flexible materials. Therefore, these 
designs are most suited for morphs that possess high surface tension, such as span, chord-wise morphing, and control surface substitution.

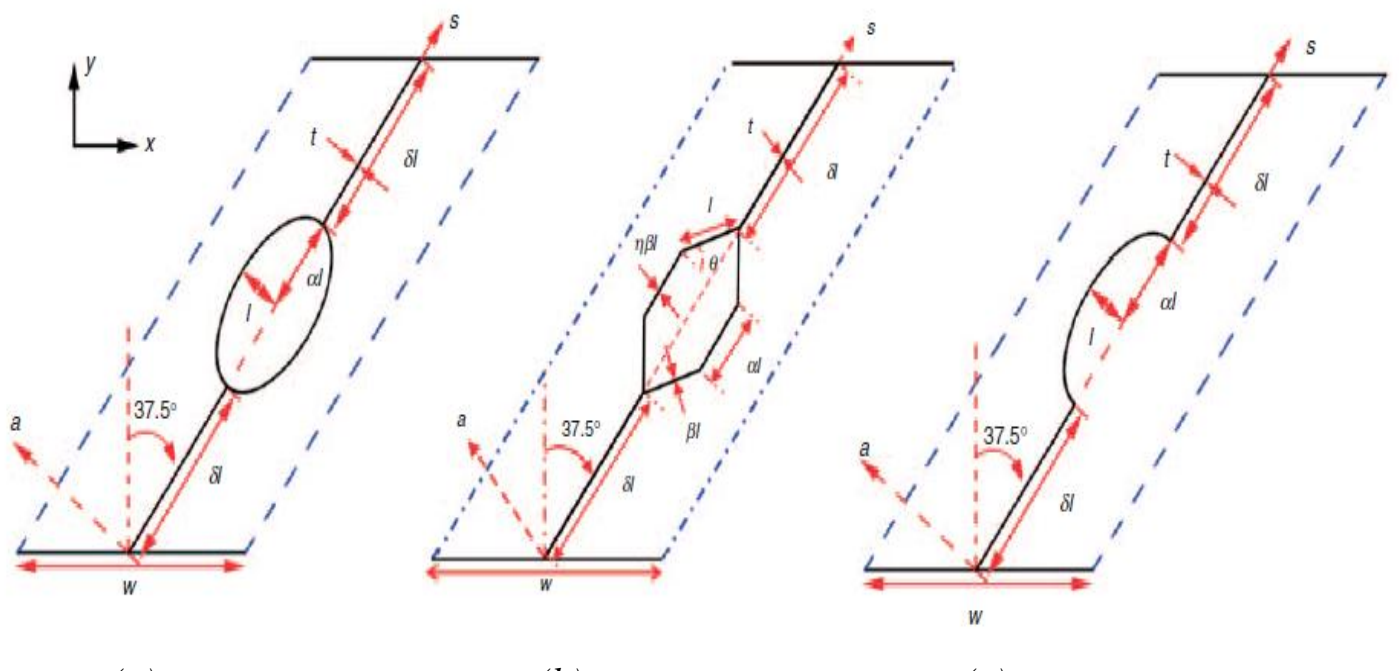

(a)

(b)

(c)

Fig. 4: (a) strand with a central ellipse (b) strand with central hexagon (c) strand with central half ellipse, all adopted from [8]

\subsubsection{MECHANICAL}

Mechanical morphing skins aims to achieve flexible motion with rigid materials in combination with some form of mechanism. Unfortunately, the higher DOF motion, the complexity of the mechanism will increase. While a high level of stiffness is ensured, an increase in weight is expected.

\section{CORrugated LAMinates}

A common morphing skin design is composed of corrugated laminates, usually reinforced with foam, sandwiched between composites. These designs were developed and tested for chord-wise and/or camber morphing. Since these designs usually possess low unidirectional stiffness, it is only applicable towards low speed small air vehicles with single DOF morphing[33, 37]. 


\section{Collapsible Structures}

An example of collapsible structures is inspired by the biomimicry of butterfly feeding tubular coil organ, emulating proboscis motion. This is employed mostly on space vehicles. A more widely applicable form of collapsible structures is folding structures, which are employed by aerial and space vehicles. Inspired from origami, folding structures have been observed as a viable option for morphing skin design. However, the implementation of folding structures on a wing is difficult, since there will be no smooth transition during deployment.

Origami, as the name suggests, is the Japanese art of folding paper into decorative shapes and figures. This is achieved through a combination of pre-folds, crease lines, and crease angles. Tomohiro [38] has developed a computer based interactive simulation of a rigid origami model. A simulation used to simulate origami's crease and fold patterns. The general purpose of the program is to provide comprehensible representation for users to understand the structure of origami based on different crease and fold patterns (Fig. 5). Given certain patterns of folds and crease angles, certain motions and shapes of morphology may be achieved.
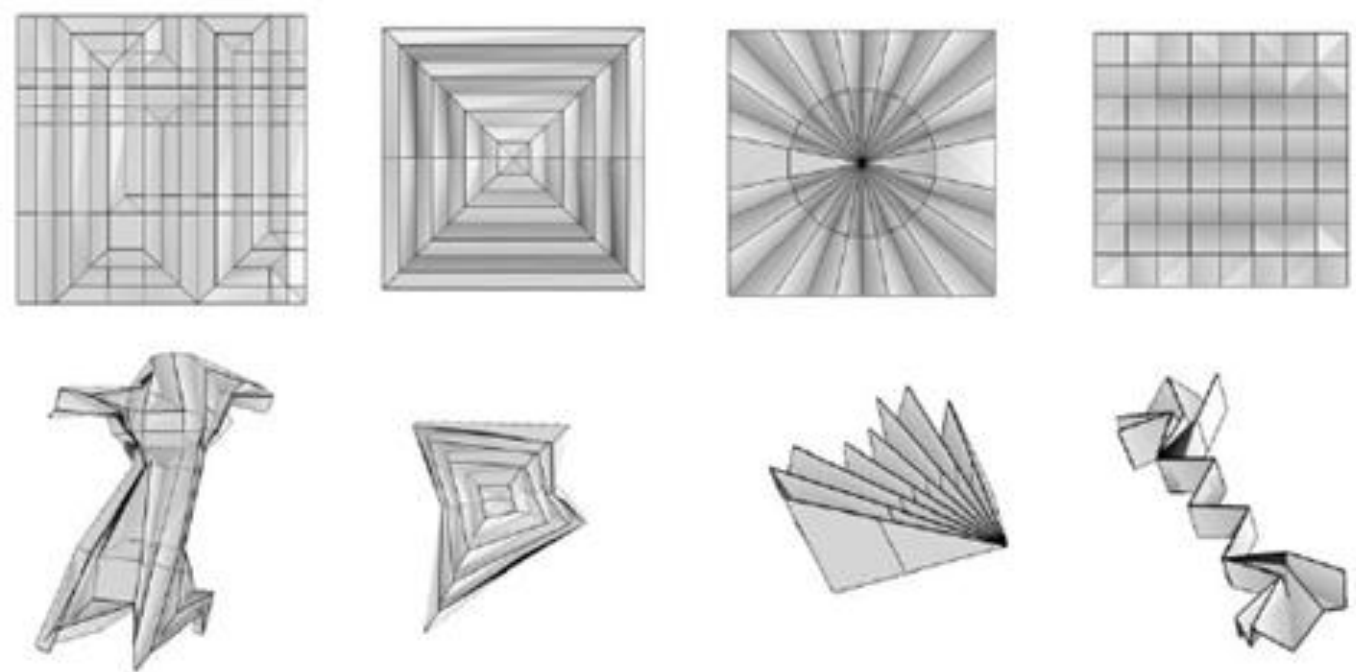

Fig. 5: Sample of different origami patterns adopted from [38] 
For example, Gattas et al [39] adopted a fundamental origami pattern, the Miura-ori, to develop a plate-based sandwich mechanism. As seen in Fig. 6, the mechanism consists of three layers, one core layer to define the deployed geometry, and two layers to maintain either the deployed form or the sandwiched form. The paper examines the physical properties of their rigid-foldable morphing mechanism. The Miura-ori pattern allows rigid panels to rotate about hinged creases without twisting or stretching of panels.

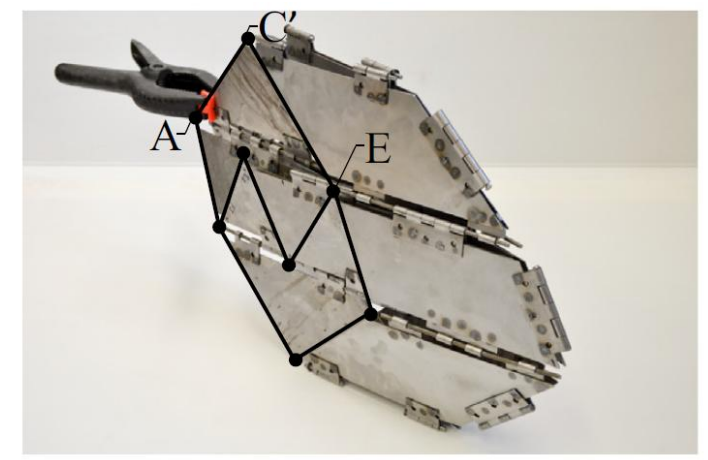

(a)

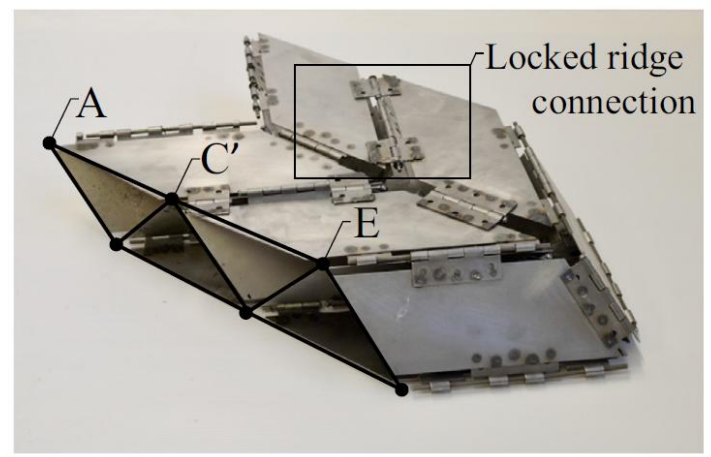

(b)

Fig. 6: (a) collapsed flat-folded structure (b) fully deployed structure, all adopted from [39]

Kirigami is a variation of origami that employs both cutting and folding. This is done to achieve collapsible structure with reduced volume. Saito et al [40] manufactured and numerically evaluated a composite wingbox comprised of a cellular hexagonal morphology for its torsional stiffness. With the application of kirigami, the overall wingbox has limited DOF of morphing, such as control surface morphing. The paper presents a kirigami manufacturing technique that allows for generating arbitrary shapes of cellular structure. Therefore, instead of developing a high-DOF morphing structure, the cellular kirigami morphing structure may be viable alternative for artificial hinge mechanisms. Kirigami is capable of higher DOF motions, as Zhang et al [41] develops a three DOF worm robot (Fig. 7). The kirigami-inspired worm robot 
imitates peristalsis and inchworm-like motion. The three DOF motions include contraction, bending, and twisting motion. The overall structure is actuated with SMA coil springs. Kirigami was implemented to replace conventional revolute, universal joints, etc. with compliant mechanisms of the folded structure. As a result, a single worm module is comprised of a single sheet of strategically sliced and folded cardboard, creating a lightweight three DOF robot.

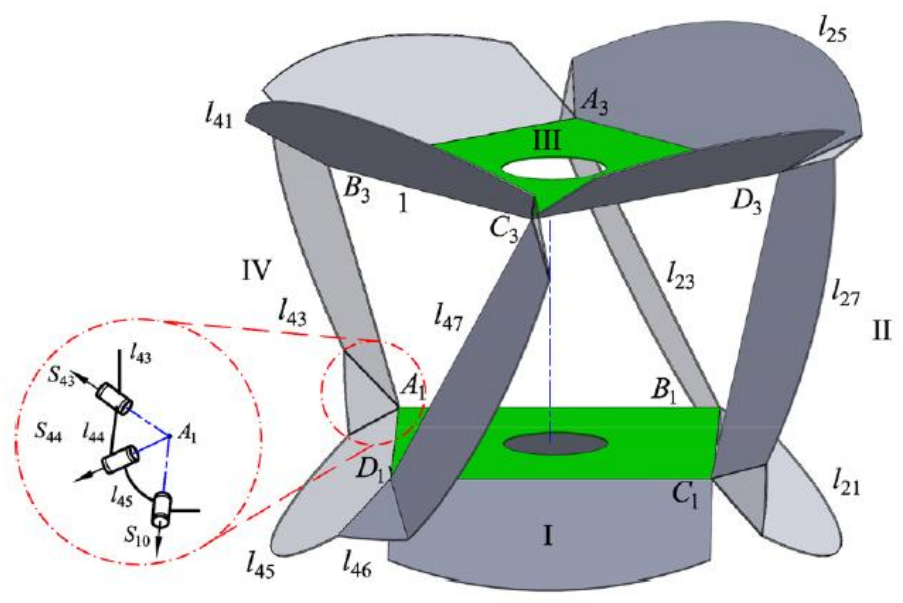

Fig. 7: Schematic kirigami structure for a single module adopted from [41]

\section{INFLATABLE STRUCTURES}

One of the appealing aspects of inflatable wing structures is its high packing efficiency. Ideally, this is used in combination with small UAVs, alluring high military interest. Wings may be inflated mid air after launch; in addition, a smooth uniform surface provides ideal aerodynamic performance, resulting in low fuel consumption. However, the high deformity of inflatable aerodynamic surfaces limits the viable operational conditions to low Reynolds numbers. Also, high aspect ratio inflatable wings require high pressures (50-100 [psi]) to bear root bending moments, this causes problems to the inflatable material and vehicle certification $[32,42]$. 


\section{Cascading Structures}

Cascading structures comes in multitude of forms. The most notable ones include overlapping and telescopic structures. Most span morphing structures employs telescopic booms or wings, generally to increase aspect ratio. Despite the benefits of high aspect ratio wings, manoeuvrability and cruise speed are sacrificed. Aerodynamically, span morphing will benefit the lift drag ratio and range, at the same time the increase in span will considerably increase the wing-root bending moment. From an aeroelastic perspective, structural deformation is amplified with the increase in span [21]. Therefore, one of the main design considerations for variable span morphing wings is the ability to sustain bending moments. Most common designs utilize a telescopic mechanism or span-wise actuation of an entire wing with flexible composite skin. The telescopic mechanism divides a wing into multiple sections and although the benefits of variable spans are achieved, such design greatly deteriorates the structural strength of the wing. Thus, an aircraft with telescopic wings is limited to low speed operations $[22,25,26]$.

\subsubsection{STIFFNESS TAILORING}

Most of the available deployable structures are developed with the main purpose is to induce large increases in surface area or with collapsibility for storage. Therefore, an alternative to shape change for morphing wing is modifying material properties. Material properties are optimized and modified with respect to specific shape criterion. There are two conventional methods: stiffness tailoring the material to achieve shape morphing and shape morphing with material that has the ability to change in stiffness. 


\section{FibER MODIFICATION}

Stiffness tailoring is achieved by optimizing material strength and stiffness in different directions, such as varying fiber/matrix orientations of composites to compensate for the morphing structure and external loading conditions. By doing so, in-plane transformation is achieved with minimal force while reducing out-of-plane transformation. Four different fiber modification processes were documented. The first process is by aligning the matrix and fibers in specific directions to achieve a highly anisotropic composite for one-dimensional morphing. An example is a fiber matrix composite (Fig. 8), the fiber dominated direction runs chord-wise and matrix dominated direction runs span-wise [43].

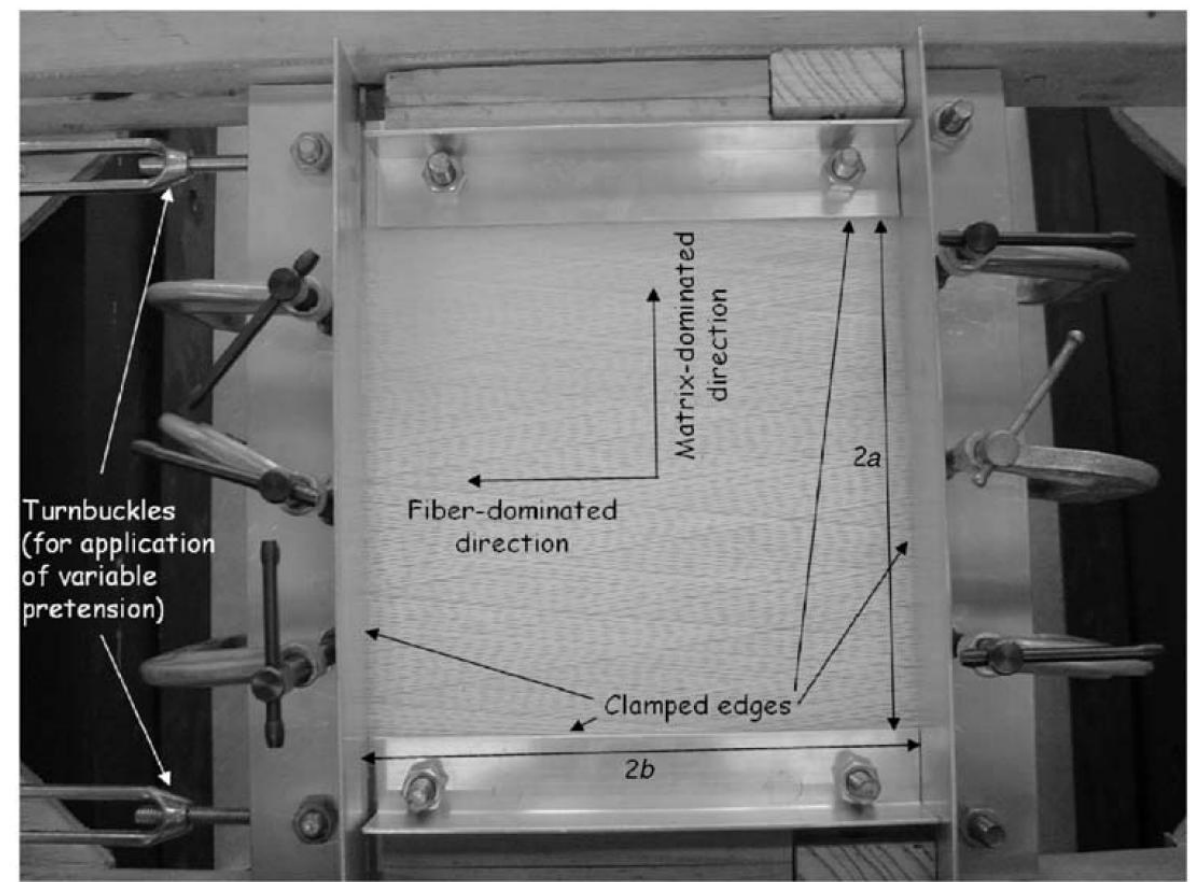

Fig. 8: Custom testing apparatus for fiber matrix composite adopted from [43]

An ideal highly anisotropic composite should have low in-plane stiffness and high out-of-plane stiffness, allowing efficient morphing. Extreme anisotropic materials are materials that possess large variations of stiffness along two orthogonal directions. As a result, the material may be susceptible to deformation in one direction due to lesser 
stiffness and minimal deformation in the respective orthogonal direction due to higher stiffness. An example of application is morphing cambers. To allow for camber morphing, the material must be flexible in the chord-wise direction and stiff in the span-wise direction [32]. A second process is by using curvilinear fibers instead of the conventional linear fibers. This process was studied and it was suggested the alternate fiber use yielded desirable in-plane and out-of-plane flexibility. However, this design was only tested on low aspect ratio wings (max aspect ratio of 2), therefore application to different wing structures is unknown [44]. The third process varies the volume fraction of fibers within a laminate to optimize in-plane flexibility and bending stiffness. The study suggests by varying fiber spacing rather than uniform distribution, there will be a $30-40 \%$ increase of in-plane flexibility and bending stiffness of the laminate [45]. The last method introduces tailoring of stiffness distribution of the skin based on specific morphing mechanism, morphing shapes, and aerodynamic loadings. This provides a variable-stiffness skin, however only a twodimensional simulation model is available [46].

\section{Multi-STABle Composites}

Multi-stable composites exhibit properties that are both stable and unstable under different states, hence allowing for potential morphing applications. To switch between states, a certain force is required as catalyst to accentuate the process. However, the forces required to initiate deformations of multi-stable composites are far too low compared to aerodynamic forces it may potentially encounter. In addition, there is high uncertainty on how the manufacturing process of the composites will affect the overall multi-stability properties [32]. 


\section{SEGMENTED STRUCTURES}

Segmented structures are bio-inspired structures from fish skins. The component of a fish skin consists of multiple discretized elements, fish scales. While in motion relative to adjacent panels, it creates a deformable structure. The underlying muscle of a fish is highly deformable, but does not provide enough stiffness to maintain a shape. However, in combination with scales, which provide high stiffness, a deformable yet stiff structure is achieved. Rediniotis et al [47] have designed and tested a highly manoeuvrable bio-inspired aquatic vehicle (Fig. 9). The robot is designed to imitate the swimming motions of a fish, with the addition of replicating the anatomical structure of a fish. The overall structure is segmented into six modules, similar to a skeletal structure of an aquatic animal. Smart memory alloy actuators are connected between modules to simulate muscle contractions to drive the modules individually. It was concluded the system had very responsive SMA controls, high deformable capability, and high manoeuvrability.

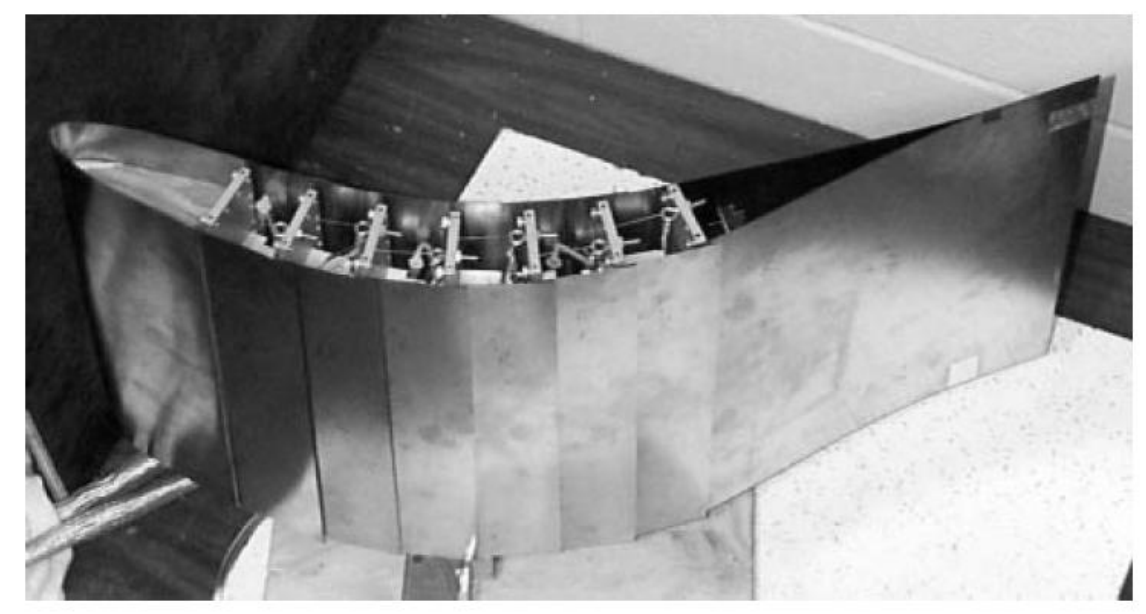

Fig. 9: Hydrofoil under deflection adopted from [47] 


\subsubsection{Stiffness Change}

Contrary to optimizing the stiffness to certain morphing conditions, an alternative method is to design materials with the ability to change stiffness. Hence giving more flexibility to various shape changes, allowing shape morphs to a higher DOF. A common approach is utilizing skin materials that have high elasticity, while maintaining sufficient stiffness to provide structural integrity as a conventional skin panel would. Within the available documentation, the skin panels are generally composed of highly anisotropic composites [48-50] or Shape Memory Polymers (SMP) [51, 52]. SMP are capable of "memorizing" shapes and alternate between them with the change in temperature. As mentioned, this design is only capable to morphing between two shapes, limiting the desired morphing DOF.

\section{SHAPE MEMORY ALLOYS AND POLYMERS}

The general shape-memory effect (SME) caused by the change in temperature is known as thermally induced shape-memory effect. For shape memory alloys (SMA), when a critical temperature is achieved, usually when the material reaches martensite phase, it will exhibit low stiffness and can be deformed easily. In order to reverse the shape change, the material is heated above its austenite temperature. This is called one-way SME. To assign or reassign memorized shapes, the material is annealed approximately above $500{ }^{\circ} \mathrm{C}$. A two-way SME is simply the ability of a material to switch between two configurations when heated and cooled. For shape memory polymers (SMP), when it is heated above the glass transition temperature, it softens and allows for temporary deformation from applied loads. When cooled, the temporary shape is retained and the loads are removed. To recover the original permanent shape, the SMP is heated above the glass transition temperature again [32, $51,52]$ 


\section{FLEXIBLE MATRIX COMPOSITES AND PNEUMATIC ARTIFICIAL MUSCLE}

Flexible matrix composite (FMC) actuators and pneumatic artificial muscle (PAM) actuators share similarities in morphing. Both are actuated with fluid to alter stiffness and manipulate shape. Due to PAM actuator's fast responsive nature, it allows for more compliant motion that regular gear and joints cannot achieve. In addition, PAM has high force to weight ratio and is easy to operate due to lenient pressure requirements. However, there is restricted motion to PAM actuators; they may only generate high forces with low displacements or low forces with high displacements. FMC actuators are a more recent alternative to PAM actuators. FMC actuators aim to achieve stiffness and shape changes directly from the structure. This is achieved by embedding FMC tubes into an elastomer matrix. Similar to PAM, the expansion and contraction of FMC tubes are regulated by fluid pressurization. Given its customizable nature, FMC actuators may orientate in greater magnitudes in comparison to PAM actuators [32]. Chen et al [53] developed and tested a camber morphing skin comprised of pneumatic muscle fibers embedded within silicone rubber matrix (Fig. 10). Therefore, instead of having a separate morphing mechanism and morphing skin, both were combined as a single system. When pressure is applied, this design is only capable of unidirectional contraction and expansion, where transverse stiffness is adjusted through pneumatic regulation.

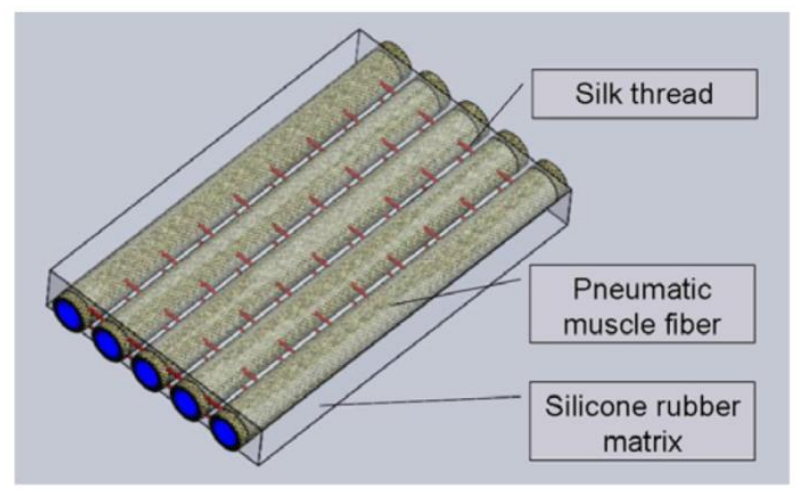

Fig. 10: Illustration of PAM morphing skin adopted from [53] 


\subsubsection{MORPHING SKIN COMPARISON}

In this chapter, the pros and cons of each aforementioned morphing skin method will be discussed. This will lead to the final verdict regarding the selection of sliding panels as Ryerson University's morphing skin design.

\section{FLEXIBLE}

The main advantages of a flexible skin are its weight, high versatility, smooth material surface, and simplicity of design. Generally, a morphing wing that uses a flexible skin must already have an underlying structure that is capable of withstanding most of the aerodynamic loads incurred during flight. As a result, the primary function of the flexible morphing skin is to produce an aerodynamic shape and maintain smooth morphing transition, while reducing the overall weight of the system. However, flexible materials are not ideal aerodynamic surfaces under certain morphing configurations. For example during a sweep morph, the alternate shearing motion between elements of the underlying mechanism will cause the flexible skin to form ripples/wrinkles. This is inevitable phenomenon, therefore as mentioned previously, many studies revolve around cellular structures to provide pretension along the flexible skin to reduce ripple formation. In addition to surface deformations, the stretching of flexible material will induce extra force upon the actuators of the underlying structure. Therefore, actuation force is varied upon stages of morphing and must be calibrated to ensure proper shape generation.

\section{MECHANICAL}

The main advantage of mechanical morphing skins is the ability to bear loads and provide high structural rigidity. Contrary to flexible skins, there is no possibility of ripple formation. The drawback of using mechanical skin panels is the complexity of 
the system. When higher DOF morphing is desired, the overall complexity of the system will increase. As a result, an increase in weight will occur, manufacturability will be an issue, and complications in kinematics of the structure will occur. As seen previously, for camber morphing, corrugated sandwich structures require complex and specific manufacturing procedure. Origami and kirigami inspired structures require multiple elements, hinges, and layers to achieve said shapes. Even a simple case of span morphing possesses bending moment complications in design. For example, telescopic structures deteriorate in structural integrity as span increases. Currently, there are no studies available regarding mechanical skin designs that operate beyond four DOF, which implies mechanical morphing skins that morph to five DOF or higher is revolutionary.

\section{STIFFness TAILORING AND STIFFness Change}

Stiffness tailoring is advantageous when small loads are required to make large deformations to the skin material, while being able to bear loads. One appealing aspect is its high customizability. Fiber orientations can be defined by the manufacturer to achieve low in-plane stiffness and high out-of-plane stiffness. As seen, it is a very complex procedure. In addition, stiffness tailored material have very limited DOF morphing, often limited to one or two DOF. Similarly, SMA and SMP have versatile morphing capabilities, due to its ability to "memorize" shapes and bear loads once the transition is complete. However, the memory effects also have limited capacity, with the current maximum of two morphing shapes and the original shape. Therefore, SMA is more commonly used as actuators. Of all the examples presented, the bio-inspired hydrofoil [47] is the most outstanding. It is a mixture between mechanical skin, stiffness tailoring, and stiffness changing material. Due to this combination, a highly manoeuvrable and rigid morphing structure is developed. 
Although the morphing range is below four DOF, it serves as a good reference for our final morphing skin selection.

\subsubsection{FinAl SELECTION}

The final morphing skin selection we have chosen at Ryerson University is the sliding panel. A physical prototype of the model may be seen below:

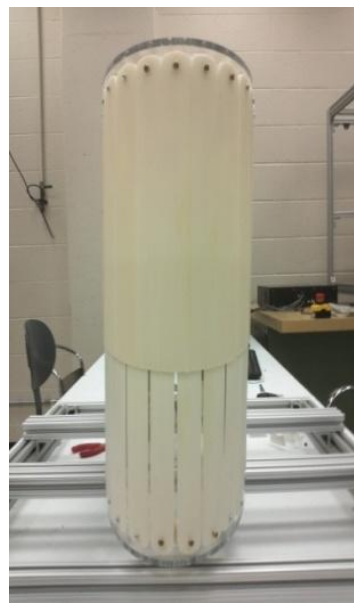

(a)

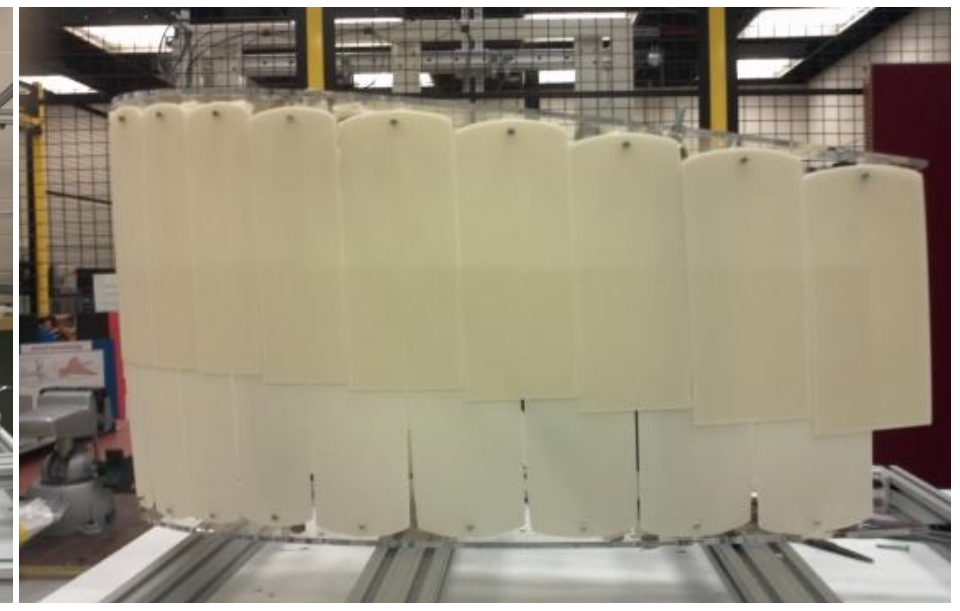

(b)

Fig. 11: (a) Sliding panel prototype leading edge view (b) Top view, all adopted from [6]

The approach to the design is to discretize a skin panel into multiple elements. As discussed in the VGTM modularity morphing wing design, discretization provides the advantage of achieving high DOF morphing. A single sliding panel element is composed of a two telescopically attached rigid panels; to ensure panels do not slide out of formation. The bottom telescopic panels are sandwiched between two top telescopic panels, with extended S-joints. The pattern is repeated along the airfoil geometry. This will allow the internal VGTM mechanism to determine the overall morphology of the skin panels. This design was selected for its structural rigidity, robustness, and versatility. Structural rigidity and robustness of system is achieved with the use of rigid material and simple flat, telescopic, panel mechanisms. 
Versatility is achieved with the combination of telescopic skin panels and extra links at the base and platform of the mechanism. This will allow for six DOF motions, while containing the internal mechanism. A few issues arise with this design. First is collision among adjacent panels during large morphing ranges. Second, there is high structural complexity of this system. Third, there is no simulation or documentation for the panel interaction among panels of this or similar design. Therefore, this thesis aims to provide proper numerical formulations for the interactions of sliding panels and suggest solutions to the colliding panel issue. 


\section{CHAPTER 3 Kinematic MODELING OF AN}

\section{ACtive Panel}

The system used in this thesis is a reconfigurable hexapod that possesses six DOF motion. The orientation and motion of the robot is controlled by six individual branches. Each branch consists of universal-prismatic-spherical structure, where the prismatic joint is active. Each branch is attached to the vertices of the equilateral hexagon base and platform. The universal joint is located at the base, with the first

rotational axis constrained tangentially to the circumference of the hexagonal geometry. The second rotational axis is perpendicular to the first, therefore passing through the center of the base. Evidently, the S-joint will be located at the platform vertices. This arrangement will allow for a six DOF parallel robot.

\subsection{MechanisM System Modeling}

Fig. 1 (a) illustrates a panel enclosed hexapod, for a single panel. The global frame $O_{b}-x_{b} y_{b} z_{b}$ is fixed to the base; a local frame $O_{p}-x_{p} y_{p} z_{p}$ is attached to the moving platform. In addition, a local skin panel frame $O_{s i}-x_{s i} y_{s i} z_{s i}$, where subscript $i$ is the panel number.

The kinematics of the parallel robot is solved by generating vector loop equations for each branch. For the $i$-th branch, the following loop equation is formed:

$$
\boldsymbol{b}_{i}+\boldsymbol{r}_{i}=\boldsymbol{h}+\boldsymbol{p}_{i}
$$


In Eq.(1), $\boldsymbol{b}_{i}, \boldsymbol{r}_{i}$, and $\boldsymbol{p}_{i}$, are the base, branch, and platform vectors, respectively. All vectors are designated in the base-fixed coordinate frame $O_{b}, \boldsymbol{h}$ is the vector representing the position of the moving platform, $\boldsymbol{h}=\left\{h_{x}, h_{y}, h_{z}\right\}^{T} \cdot \boldsymbol{p}_{i}=\boldsymbol{R}_{p} \boldsymbol{p}_{i}^{\prime}$, and $\boldsymbol{p}_{i}^{\prime}=\left\{p_{i, x}^{\prime}, p_{i, y}^{\prime}, p_{i, z}^{\prime}\right\}^{T}$ is defined in the platform frame. The rotation matrix $\boldsymbol{R}_{p}=$ $\boldsymbol{R}_{x}\left(\theta_{x}\right) \boldsymbol{R}_{y}\left(\theta_{y}\right) \boldsymbol{R}_{z}\left(\theta_{z}\right)$, defined as:

$$
\begin{aligned}
\boldsymbol{R}_{x} & =\left[\begin{array}{ccc}
1 & 0 & 0 \\
0 & \cos \theta_{x} & -\sin \theta_{x} \\
0 & \sin \theta_{x} & \cos \theta_{x}
\end{array}\right] \\
\boldsymbol{R}_{y} & =\left[\begin{array}{ccc}
\cos \theta_{y} & 0 & \sin \theta_{y} \\
0 & 1 & 0 \\
-\sin \theta_{y} & 0 & \cos \theta_{y}
\end{array}\right] \\
\boldsymbol{R}_{z} & =\left[\begin{array}{ccc}
\cos \theta_{z} & -\sin \theta_{z} & 0 \\
\sin \theta_{z} & \cos \theta_{z} & 0 \\
0 & 0 & 1
\end{array}\right]
\end{aligned}
$$

Eq.(1) can be rearranged to solve for the displacement of the prismatic joint as:

$$
r_{i}=\left|\boldsymbol{h}+\boldsymbol{p}_{i}-\boldsymbol{b}_{i}\right|
$$

\subsection{Active Panel Modeling}

As shown in Fig. 12 (b), each skin panel is of a rectangular shape and the centerline in span-wise direction is rigidly attached to the active prismatic joint, whose length is $r_{i}$ determined by eqn. (3). Subscript $i$ is used to allocate the position of each panel and link simultaneously. From Fig. 12 (a), skin panel $i$ can be defined by four point 
vectors: $\boldsymbol{s}_{1 i}, \boldsymbol{s}_{2 i}, \boldsymbol{s}_{3 i}, \boldsymbol{s}_{4 i}$. Each point vector, denoted by $j$, can be obtained from the following equation:

$$
\boldsymbol{s}_{j i}=\boldsymbol{b}_{i}+\boldsymbol{R}_{s i} \boldsymbol{s}_{j i}^{\prime}
$$

From Eq. (4), $\boldsymbol{s}_{j i}$ is defined as the global co-ordinates for $j$-th corner on the $i$-th link. Due to the formulation of the model, $\boldsymbol{b}_{i}$ represents the starting position. $\boldsymbol{s}_{j i}^{\prime}$ is the local co-ordinates for any of the $j$-th corners of the skin panel. $\boldsymbol{R}_{s i}$ is the rotation matrix of the $i$-th panel and defined as:

$$
\boldsymbol{R}_{s i}=\left[\begin{array}{lll}
x_{s i} & \boldsymbol{y}_{s i} & \boldsymbol{z}_{s i}
\end{array}\right]
$$

Where each axis is defined as:

$$
\boldsymbol{x}_{s i}=\frac{\boldsymbol{r}_{i}}{\left|\boldsymbol{r}_{i}\right|}, \boldsymbol{y}_{s i}=\frac{\boldsymbol{n}_{i}}{\left|\boldsymbol{n}_{i}\right|}, \mathbf{z}_{s i}=\frac{\boldsymbol{x}_{s i} \times \boldsymbol{y}_{s i}}{\left|\boldsymbol{x}_{s i} \times \boldsymbol{y}_{s i}\right|}
$$

In Eq.(6), $\boldsymbol{n}_{\boldsymbol{i}}$ is the normal vector between the base and branch vector:

$$
\boldsymbol{n}_{i}=\boldsymbol{r}_{i} \times \boldsymbol{b}_{i}
$$

The general motion equation can be simplified to a vector form. The derivation of the point $\boldsymbol{s}_{1 i}$ will be used to demonstrate the procedure:

$$
\begin{gathered}
\boldsymbol{s}_{1 i}=\boldsymbol{b}_{i}+\boldsymbol{R}_{s i} \boldsymbol{s}_{1 i}^{\prime} \\
\boldsymbol{s}_{1 i}^{\prime}=\boldsymbol{r}_{i}^{\prime}+\boldsymbol{n}_{i}^{\prime}=\boldsymbol{r}_{i}^{\prime}+f \boldsymbol{y}_{s i}=\boldsymbol{r}_{i}^{\prime}+f\left[\begin{array}{l}
0 \\
1 \\
0
\end{array}\right]
\end{gathered}
$$




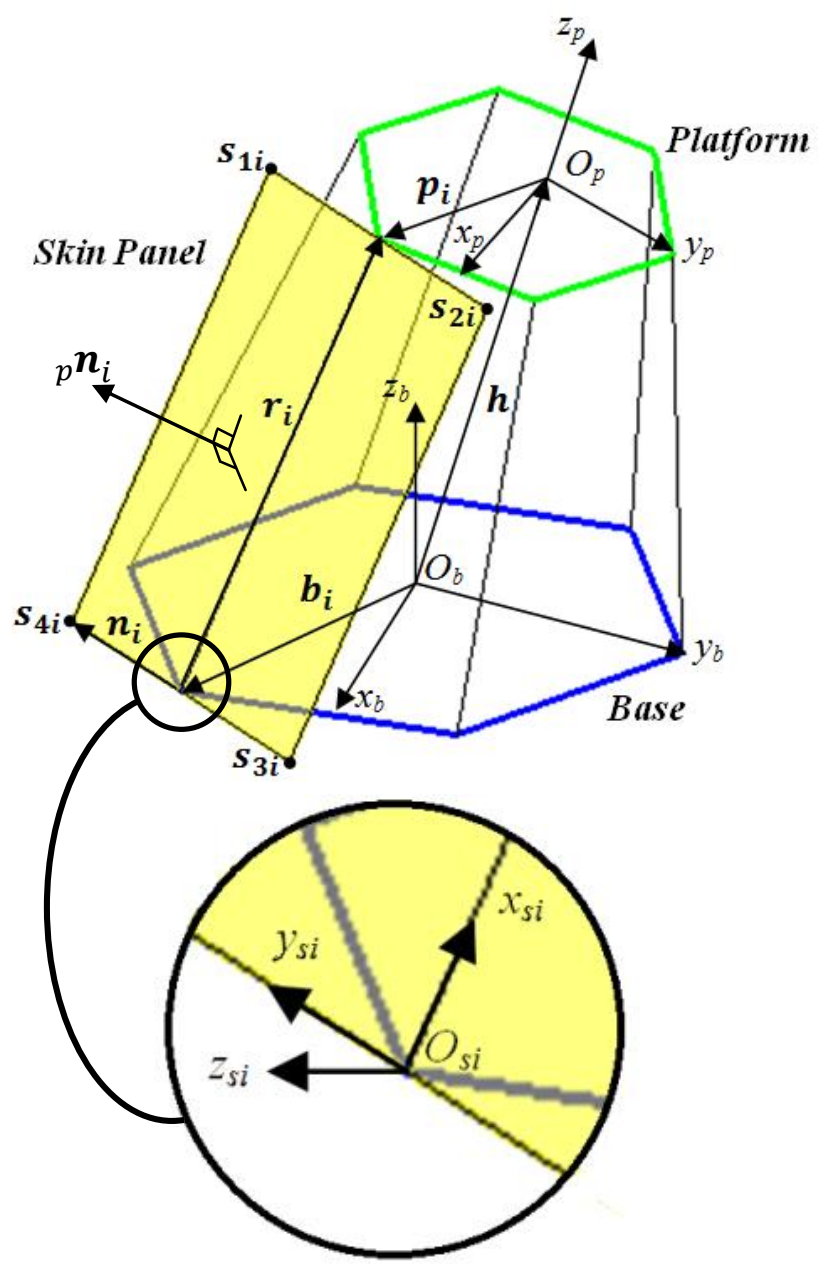

(a)

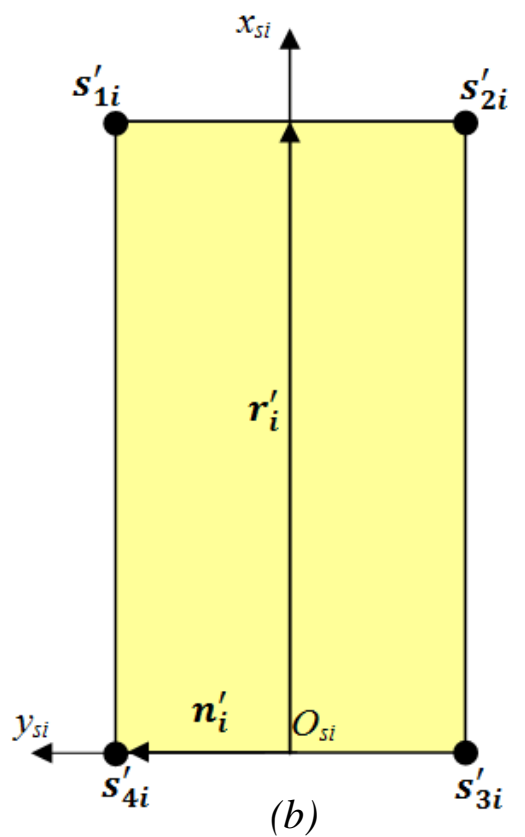

Fig. 12: (a) Definition of hexapod configuration, (b) Definition of skin panel configuration 
From Eq. (9), a scalar constant $f$ is used to adjust the width of the skin panel. From Fig. 12 (b), local point $\boldsymbol{s}_{1 i}^{\prime}$ is the vector addition of local vectors $\boldsymbol{r}_{i}^{\prime}$ and $\boldsymbol{n}_{i}^{\prime}$. Where $\boldsymbol{y}_{s i}$ is the local unit vector of $\boldsymbol{n}_{i}^{\prime}$. Given the local vectors:

$$
\begin{gathered}
\boldsymbol{s}_{1 i}^{\prime}=\boldsymbol{r}_{i}^{\prime}+\boldsymbol{n}_{i}^{\prime} \\
\boldsymbol{s}_{2 i}^{\prime}=\boldsymbol{r}_{i}^{\prime}-\boldsymbol{n}_{i}^{\prime} \\
\boldsymbol{s}_{3 i}^{\prime}=-\boldsymbol{n}_{i}^{\prime} \\
\boldsymbol{s}_{4 i}^{\prime}=\boldsymbol{n}_{i}^{\prime}
\end{gathered}
$$

The final vectors in the global frame are obtained with direct substitution:

$$
\begin{gathered}
\boldsymbol{s}_{1 i}=\boldsymbol{b}_{i}+\boldsymbol{r}_{i}+\boldsymbol{n}_{i} \\
\boldsymbol{s}_{2 i}=\boldsymbol{b}_{i}+\boldsymbol{r}_{i}-\boldsymbol{n}_{i} \\
\boldsymbol{s}_{3 i}=\boldsymbol{b}_{i}-\boldsymbol{n}_{i} \\
\boldsymbol{s}_{4 i}=\boldsymbol{b}_{i}+\boldsymbol{n}_{i}
\end{gathered}
$$

\subsection{COLLision Detection}

The collision detection (CD) proposed for the simulation utilizes a general case of surface-surface intersection (SSI) of flat panels. The solution of the collision will result in a linear intersection (Fig. 13). If a solution is present in the SSI analysis, the linear intersection will be iterated. These lines will serve as a guide for the removal of excess material. 


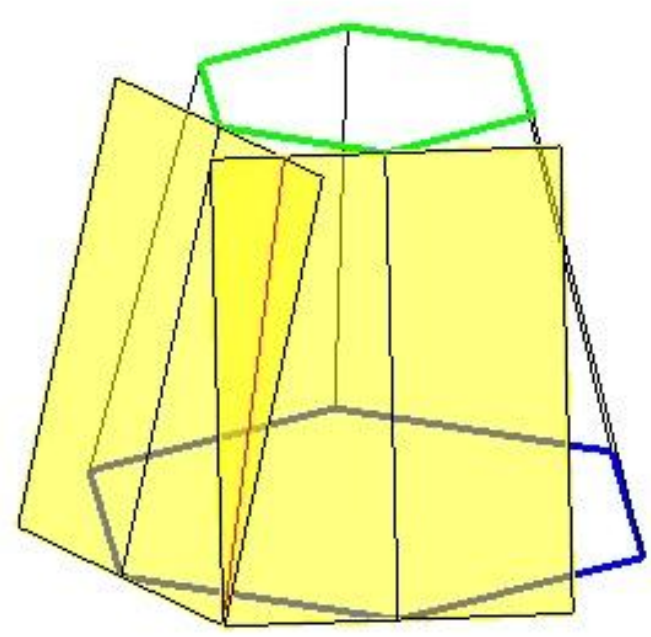

\section{Fig. 13: Example collision between two skin panels}

To analyse the interactions of adjacent panels, a collision detection method is required. There are numerous documentations available regarding the study of collision detection for a multitude of different geometries, most of which utilizes complex numerical algorithms for computation, the most common methods are bounding volume, stochastic, distance field, spatial subdivision, and image space methods. Bounding volume hierarchies break down the object into smaller partitions. Each partition is bounded by a general volume/shape to approximate and simplify iterations for collision. However, the deformation of the object will require frequent updates of the hierarchy, which may result in a loss of efficiency $[54,55]$.

Stochastic method is an inexact approximation of the actual object for collision detection. However due to inexact approximations, computation time may fluctuate based on the resolution defined by the user $[54,56]$.

Distance field is a derived representation of a digital image. It may be sign designated to indicate whether a point is inside or outside. In addition to collision detection, distance field may be used for morphing, volumetric modeling, motion planning, and 
fire animation. Although this method is applicable for $\mathrm{CD}$, its efficiency is lacking compared to other available methods. However, it is a robust method and capable of distinguishing inside and outside space [54].

Spatial subdivision or space partitioning is the process of dividing space into subsets. Similar to bounding volume hierarchies, this is a simple and fast method for CD of deformable objects. The only complication is finding a suitable data structure to represent the 3D space. Image space techniques are an emerging study for collision detection. It does not require pre-processing compared to existing methods, therefore reducing work time and highly viable for dynamically deforming objects [54].

The listed methods are applicable for rigid bodies and deformable structures, such as simulation of fabric. However, in this study, flat rigid panels are used. Therefore, a more straightforward approach was taken by utilizing the most basic form of collision detection - surface-surface intersections (SSI). This is a popular research topic within the studies of mathematics, CAD systems, computer science, and robotics.

There are three popular methods for SSI: lattice evaluation, marching, and subdivision method $[57,58]$.

Lattice evaluation method employs dimension reduction to eliminate random variables and accelerate the process during computation. This is accomplished via grid generation of the differential surface, where intersections of isoparametric curves between bisecting surfaces are computed. However this method is highly dependent upon proper selection of grid resolution, poor resolution may result in the loss of detail. Also depending on the grid resolution, it may require vast amounts of computational resources $[59,60]$. 
Marching method is a common numerical method to solve for boundary value problems. Given a surface, an approximate boundary is defined around it. The boundary is defined with a sequence of points. When the boundary does not satisfy the conditions of the iteration, it will "march" in an incremental step size along a direction defined by the differential surface. Although the method is robust for determining the intersections between two surfaces, the method lacks the algorithm to determine the initial starting condition. Hence, lattice evaluation or subdivision method is used to achieve that. In addition, similar to the lattice method, an incorrect selection of step size may cause a loss in resolution, resulting in erroneous or incomplete results $[61,62]$.

Subdivision or the divide and conquer method is simplest method out of the three. The method simplifies differential surface into simpler problems such as multiple flat plane-to-plane intersections, creating a subset of problems that can be solved directly. The results are then concatenated to form the final solution. Contrary to the marching method, it does not require an initial condition. However, there is no direct method of regulating the resolution of the results, which may cause a loss in results for highly detailed intersections $[58,63]$.

The aforementioned methods are all applicable for static cases. In the case of the presented morphing structure, a dynamic model is used. Although the system is dynamic, SSI methods are still applicable. The dynamic motion of the morphing structure can be discretized into multiple static cases, where SSI can be performed individually. In addition, the SSI method utilized will be in the simplest form, for it does not have a complex shape, only flat rectangular panels. This results in a plane-toplane intersection problem. 
To begin the SSI procedure, individual panels must be defined as numerical functions in order to iterate for intersections. The panel equations may be defined with any three points defined in Fig. 12 (b). For this case, $\boldsymbol{s}_{1 i}, \boldsymbol{s}_{2 i}, \boldsymbol{s}_{3 i}$ are selected. The plane function of a single skin panel is defined by the following:

$$
\begin{gathered}
{ }_{p} \boldsymbol{n}_{i}=\left(\boldsymbol{s}_{1 i}-\boldsymbol{s}_{2 i}\right) \times\left(\boldsymbol{s}_{1 i}-\boldsymbol{s}_{3 i}\right) \\
{ }_{p} \boldsymbol{n}_{i}^{T} \cdot\left(\left[\begin{array}{l}
x \\
y \\
z
\end{array}\right]-\boldsymbol{s}_{1 i}\right)=A_{i} x+B_{i} y+C_{i} z+D_{i}
\end{gathered}
$$

Eq. (13) provides a symbolically defined plane function, which can be expanded into a general plane equation. Where ${ }_{p} \boldsymbol{n}_{i}$ is the planar normal vector of the respective skin panel. The overall function is governed by global $x, y$, and $z$ co-ordinates. With all planar equations determined, intersections among the adjacent panels are evaluated. The intersecting line function, denoted as $\boldsymbol{l}$, will be defined in the vector form of:

$$
\boldsymbol{l}_{i}={ }_{i n t} \boldsymbol{q}_{i}+{ }_{i n t} \boldsymbol{n}_{i} t_{\text {int }}
$$

In the above equation, ${ }_{i n t} \boldsymbol{n}_{i}$ is the directional vector of the line and $t_{\text {int }}$ is for function parameterization. $t_{\text {int }}$ varies from negative infinity to positive infinity. ${ }_{\text {int }} \boldsymbol{q}_{i}$ is the default starting position of the line, this can be defined from equating for the solution of two skin panels. Given that, there are two equations and three unknowns. An initial condition of $x=0$ (assuming there is a solution through the $\mathrm{x}-\mathrm{y}$ plane) was used to achieve a determinate system and both equations are then equated to zero. Note that there are six skin panels, each equated with the subsequent adjacent panels. Therefore to maintain counter $(i)$ consistency, the sixth panel will be solved against the first panel: 


$$
{ }_{p} \boldsymbol{n}_{i} \cdot\left(\left[\begin{array}{l}
x \\
y \\
0
\end{array}\right]-\boldsymbol{s}_{1 i}\right)={ }_{p} \boldsymbol{n}_{i+1} \cdot\left(\left[\begin{array}{l}
x \\
y \\
0
\end{array}\right]-\boldsymbol{s}_{1 i+1}\right)=0
$$

Due to the orientation of the robot, the above assumption of $x=0$ is valid. Since the line of intersection will never be parallel to the $\mathrm{x}-\mathrm{y}$ plane, it will eventually pass through the $\mathrm{x}-\mathrm{y}$ plane at a given $t_{\text {int }}$.With the intersection line defined, the points of the line that lie on the skin panel must be determined. Only two points will be required, the start and end point that intersects the edge of panel. These points are later transferred onto the skin panel's local plane for the slicing algorithm. To achieve this, each edge of the skin panel were converted into vector line functions, similar to the intersecting line function. The edge of each skin panel will be denoted as $\boldsymbol{g}$.

$$
\boldsymbol{g}_{j i}=\boldsymbol{s}_{j i}-\left(\boldsymbol{s}_{j+1, i}-\boldsymbol{s}_{j i}\right) t
$$

The above equation will equate all edge functions of the $j$-th edge on the $i$-th panel. $\boldsymbol{s}_{j i}$ is used as the default starting position and $\left(\boldsymbol{s}_{j+1, i}-\boldsymbol{s}_{j i}\right)$ is the directional vector of the line. In this case, $t$ is used to parameterize the equation, note that $t$ and $t_{\text {int }}$ are not equal. Similar to the intersection calculations, counter consistency $(j)$ is maintained. Given that there are only four edges for each panel, the directional vector of the 4-th edge will be equated from $\boldsymbol{s}_{1 i}$ to $\boldsymbol{s}_{4 i}$.

Once the intersection line and panel edge line functions have been defined, it is possible to equate for intersection points. These points will later define a guide for the slicing algorithm. The solution of the intersection points are obtained by equating $\boldsymbol{l}$ and $\boldsymbol{g}$ as equal. This is performed for all four edges of each for all six panels. The 
solutions obtained from the above are the parameters $t$ and $t_{\text {int }}$. Using either parameter, they are substituted into their respective line equations to obtain the global co-ordinates of the intersection line. Since $t$ and $t_{\text {int }}$ may vary indefinitely, only intersection points of $t$ and $t_{\text {int }}$ within the boundaries of the given plane are stored.

\subsection{Slicing Method}

\subsubsection{Methodology}

In this chapter, the methodology will be presented in detail. In addition, assumptions, requirements, and limitations of the methodology will be mentioned. Fig. 14 illustrates the flow diagram of the methodology. The methodology begins with the computation of the morphing range with the provided user inputs via inverse kinematics. If necessary, the workspace of the robot without the skin panel may be iterated in this step. Once the morphing range is determined, skin panel orientations are computed based on each step of the morph. Since the panels vary span-wise with a telescopic mechanism, the possibility of panel separation from insufficient panel length must be considered. To compensate for this, an overlapping criterion is added to each step of the skin panel formulation. If the top and bottom segments do not fulfill a percentage overlap, the lengths of both panels are extended span-wise until the criterion is met. After panels have been generated for all steps, it is stored and scanned through the collision algorithm. The collision algorithm will scan all panel data for each step and compute each panel's possible collision with the adjacent panels. If a collision is present, the line of intersection between the colliding panels is computed. The line of intersections are stored and then passed through the slicing algorithm. The slicing algorithm will eliminate excess surface area furthest from the center of the panel beyond the line of intersection, resulting in the final geometry. 


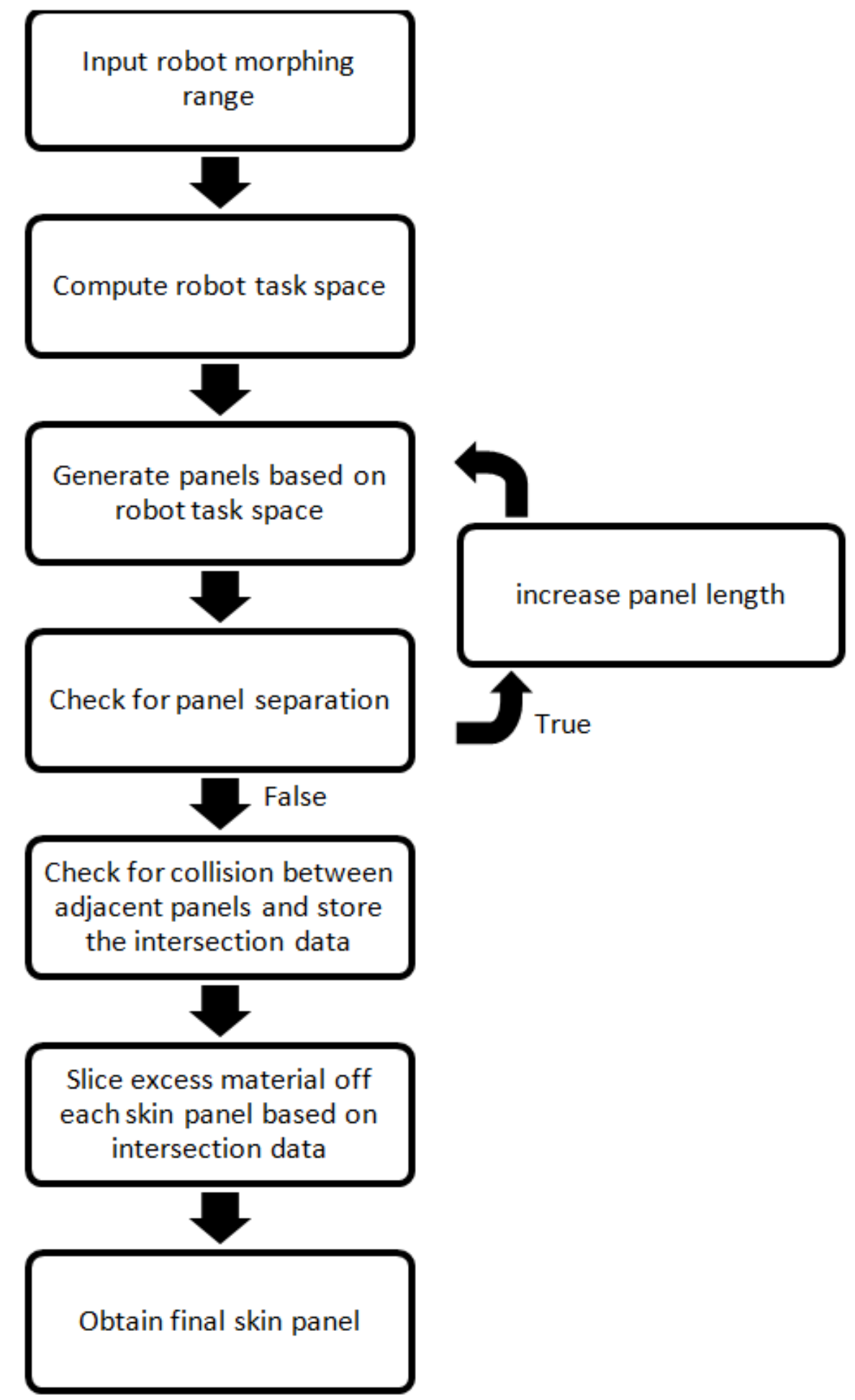

Fig. 14: Flow chart of slicing methodology 


\subsubsection{Algorithi}

The slicing algorithm eliminates excess material that is defined through collision and generates a skin panel that has the smallest possible area for a given mechanism task space.

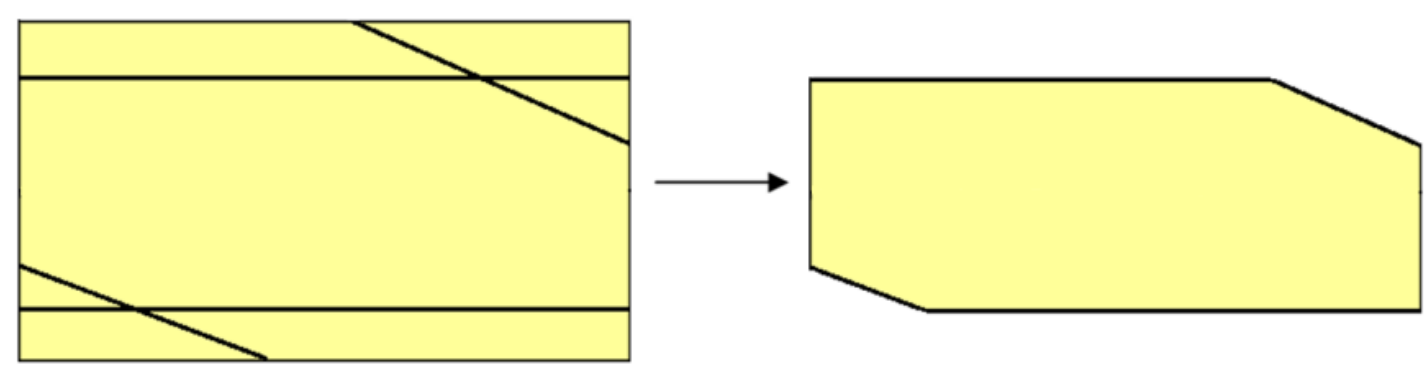

Fig. 15: Example of slicing algorithm. Left panel has two sets of collisions. Once sliced, the final geometry is obtained on the right

Fig. 15 depicts an example of the slicing algorithm. It eliminates the excess material that lies outside of the intersection, resulting in a skin panel with minimal surface area, hence the name slicing. In the diagram, more than one set of intersections is used as the slicing guide. A single set of intersection contains two line equations, one on each side of the panel. One set belongs to a z-axial twist and the other is when the robot is in idle. With reference to Fig. 12(b), to simplify the procedure of slicing, skin panelfixed frame $\left(O_{s i}\right)$ co-ordinates were used to eliminate $\mathrm{z}$ co-ordinates. The slicing algorithm will be programmed with respect to the flow diagram in Fig. 16. By using the local intersection points, a linear equation is formed consisting of only $\mathrm{x}$ and $\mathrm{y}$ values. Each y value is calculated along the x-axial span of the skin panel. Once all the y values have been calculated for all intersections, they are stored and only the smallest $y$ values are taken. The skin panel co-ordinate system is set-up for the convenience of determining the magnitude of y values. On the left of the x-axis, the smallest positive y values are saved and on the right side, only the smallest negative y 
values are saved. As a result, an array of y values is saved at the respective $\mathrm{x}$-axial span and can be used to generate the final image of the skin panel.

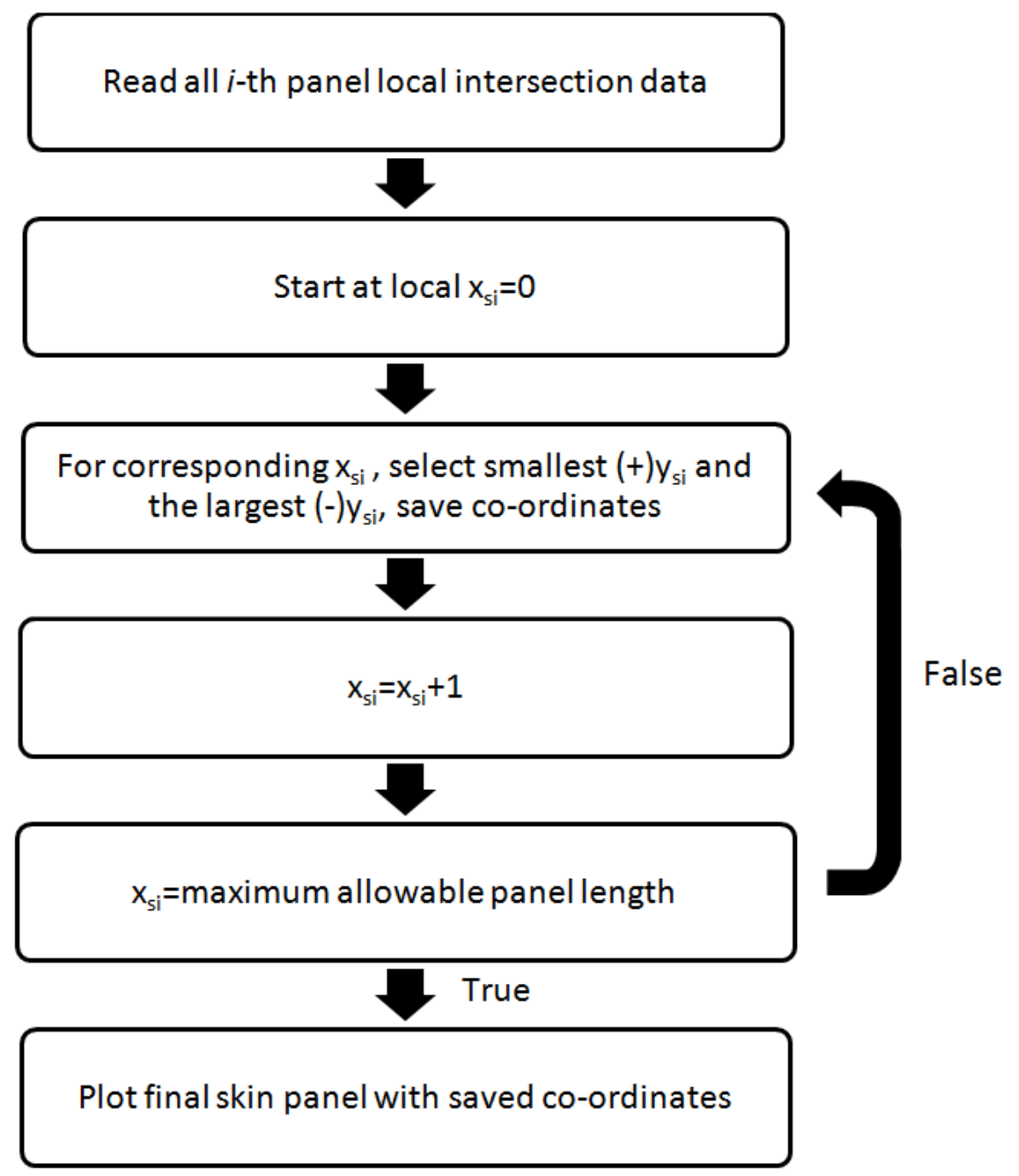

Fig. 16: Flow chart of slicing algorithm 


\section{CHAPTER 4 ACtive Panel Simulation}

In this chapter, a MATLAB simulation results are presented to validate the methodology and algorithms presented in the previous chapters. The algorithms in the methodology are formulated based on a span-wise morphing panel, which is unrealistic. However, the methodology is applicable to telescopic panels. Therefore, all results presented in this chapter are with respect to segmented panels.

\subsection{Motion AnAlysis OF THE MEChanism System}

The program allows the user to input a specified $\mathrm{x}, \mathrm{y}$, and $\mathrm{z}$ translation, along with rotations along the respective axis, therefore simulating a six DOF robot. A general visual representation is displayed below:

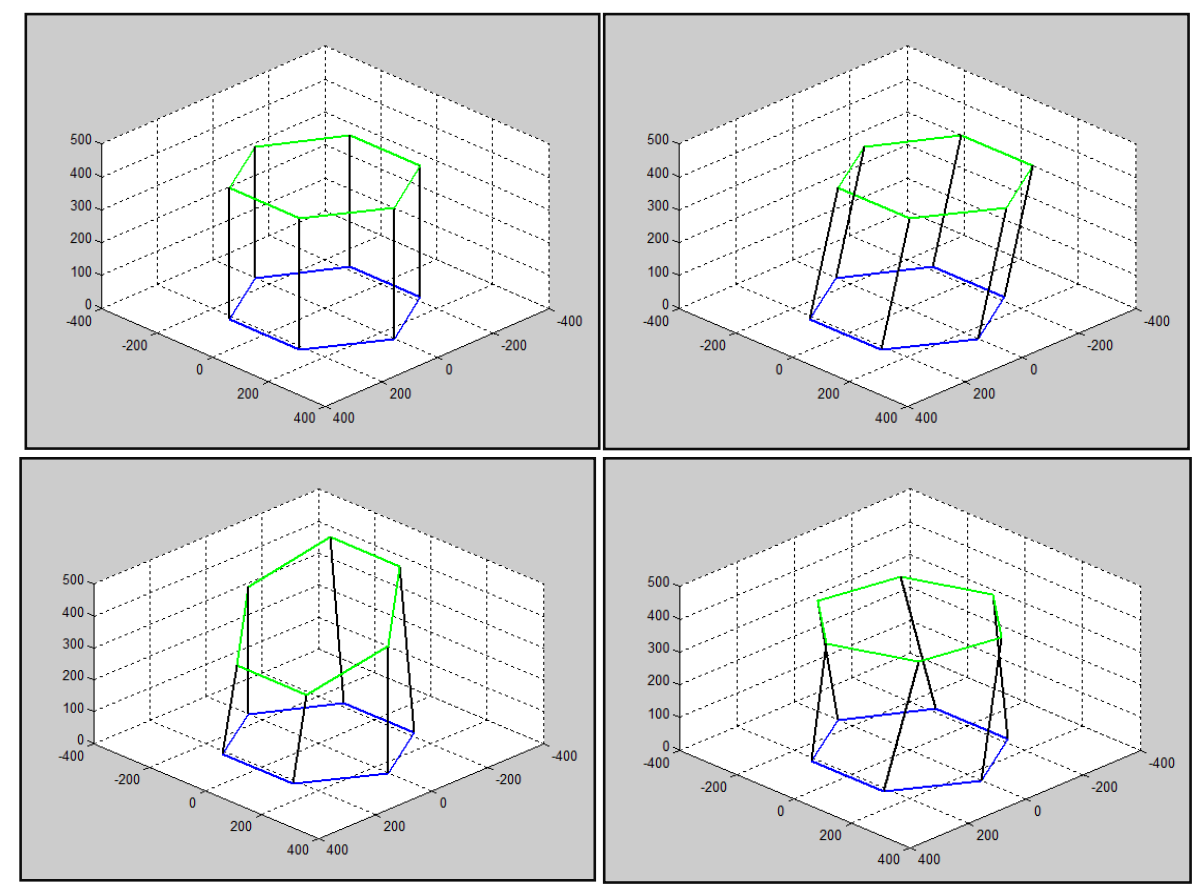

Fig. 17: Examples of hexapod MATLAB simulation

Fig. 17 depicts examples of the hexapod with different user inputs. Twelve critical points are calculated and then linked with color-coded lines to simulate the motion of a hexapod at a given condition. The blue hexagon represents the base, centered at co- 
ordinates $(0,0,0)$, and the green hexagon represents the platform. The remaining lines represent the prismatic links of the hexapod.

\subsection{Motion Analysis of Active Skin Panels}

With the same simulation, skin panels are added. A general depiction can be seen below:

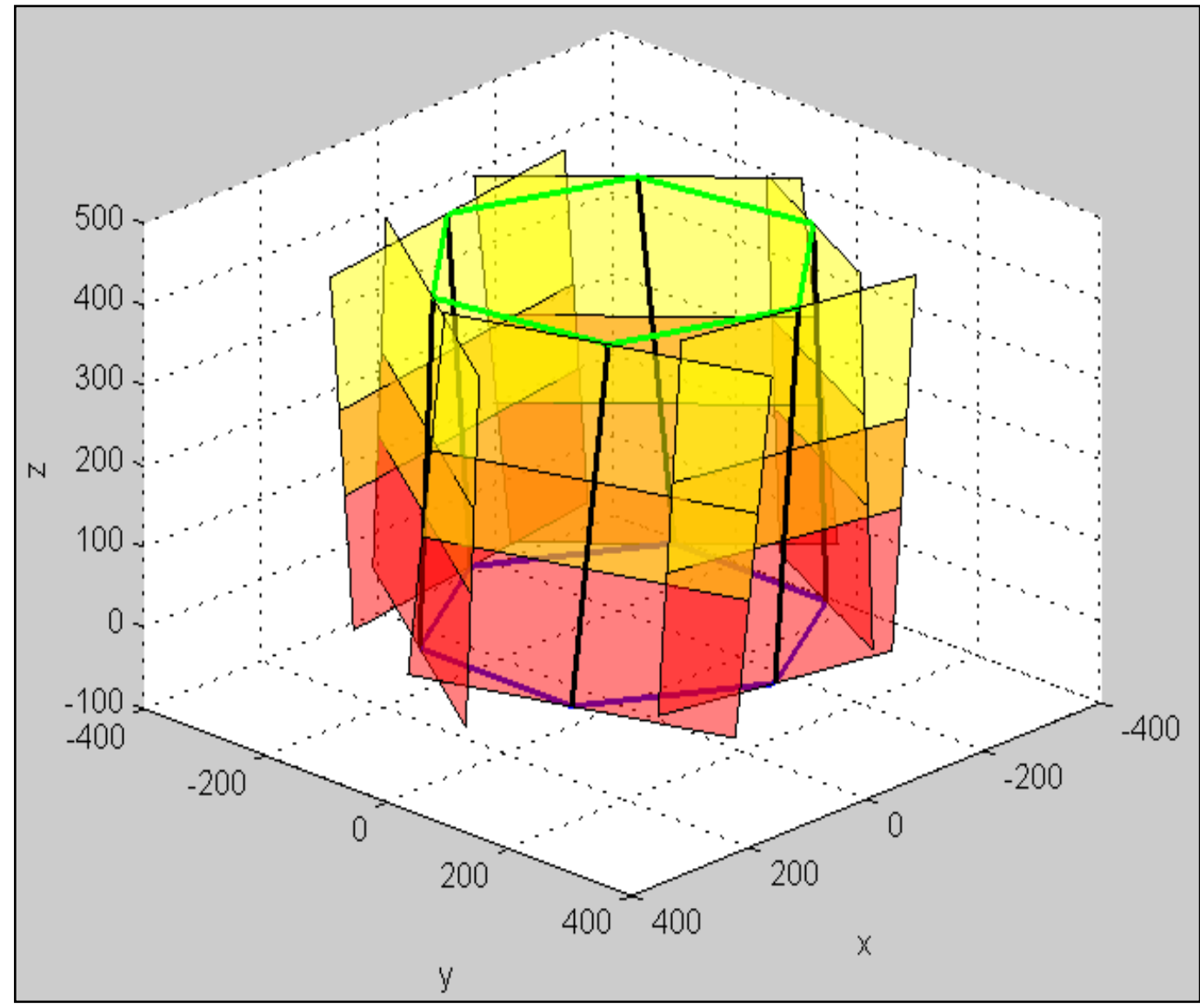

Fig. 18: Example of hexapod with skin panels. The orientation is a 10 [deg] twist around the global $\mathrm{z}$-axis

In Fig. 18, top and bottom panels are color coded for visual identification of the percentage overlap and designate the orientation of the panels. 


\subsection{COLlision Algorithm}

A general output of the collision analysis is presented in Fig. 19. All co-ordinates in this figure are in global co-ordinate system. As seen, four points of intersection are available for each panel, based on the interactions of neighbouring panels.

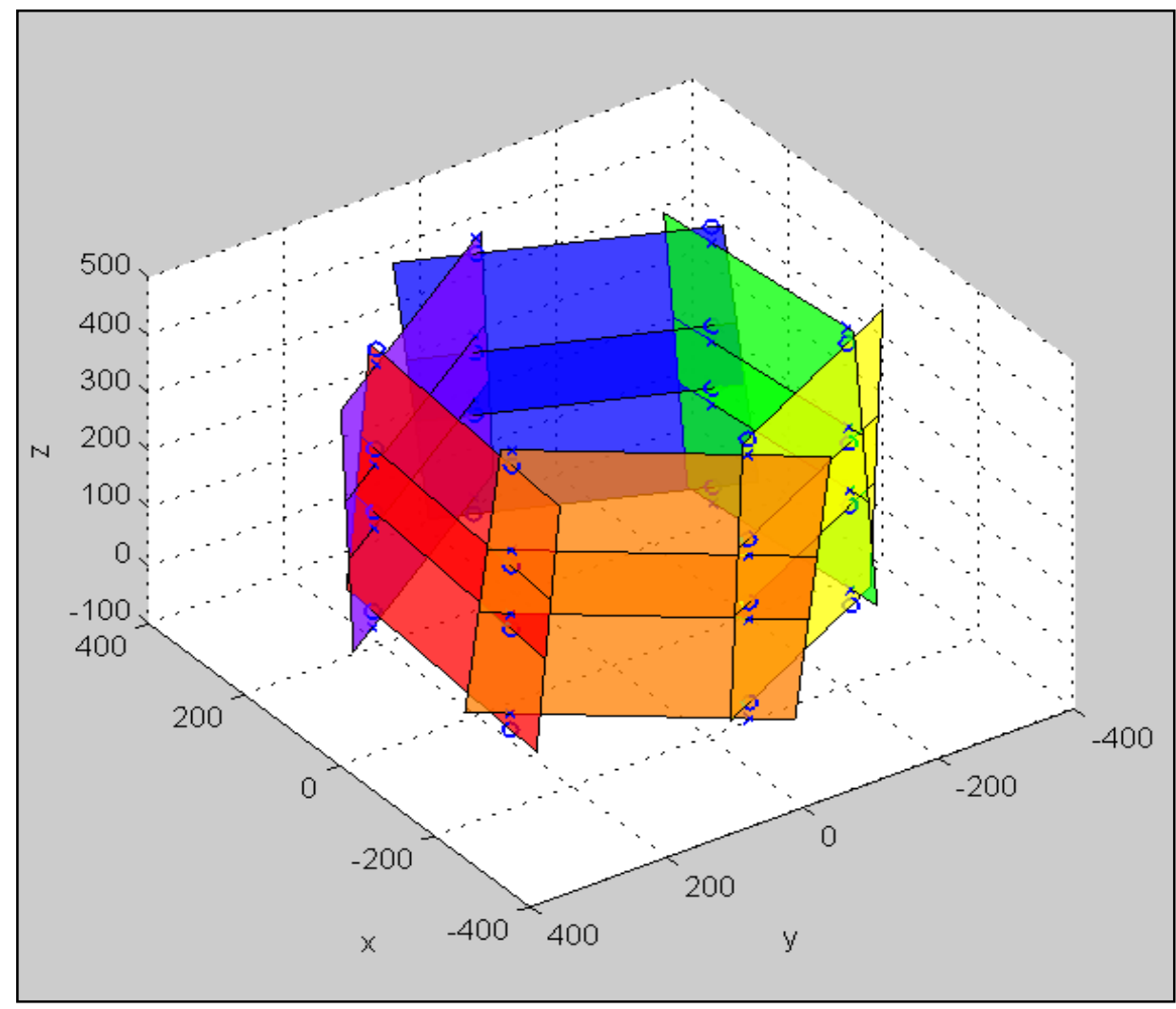

Fig. 19: Example of program output for collision algorithm. This figure shows all collision points on each individual panel

\subsection{Slicing Algorithm}

A general output of the slicing algorithm can be seen below, where the darkened area within the red dotted line indicates the final sliced shape. The figure below, are represented in its local co-ordinate system, with the lack of z co-ordinates $(z=0)$ : 


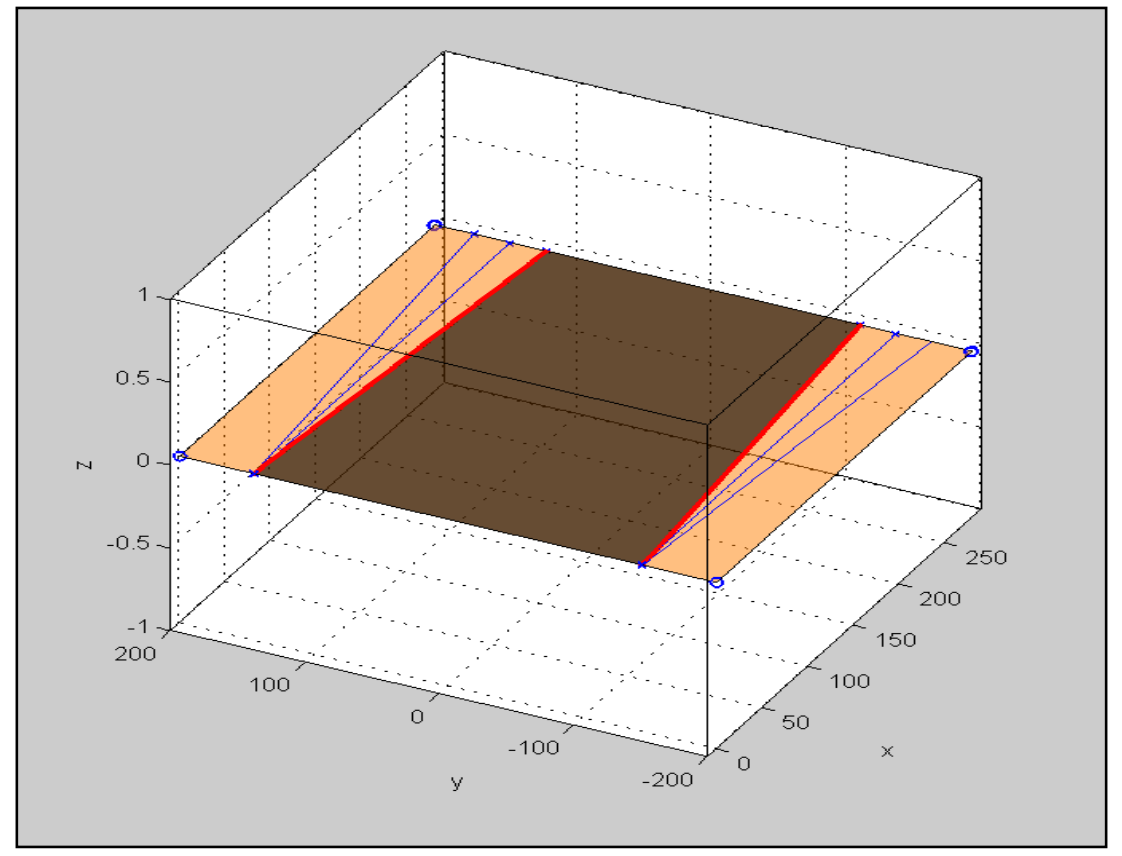

Fig. 20: Slicing algorithm result for one panel. Three different orientations have been tested on this panel and the final shape is shaded

As seen in Fig. 20, there are three sets of intersections based on different orientations. A close-up of the bottom left corner of the panel can be seen in Fig. 21. While the lines of intersection overlap each other, as shown in Fig. 21, the algorithm will select the ideal $y$-values that will provide the smallest area for the given workspace. To ensure robustness and validity of the algorithm, it was thoroughly tested on other cases with 20 or more intersections. Those of which include translations along the global $\mathrm{x}, \mathrm{y}, \mathrm{z}$-axis and rotation about the global $\mathrm{x}, \mathrm{y}, \mathrm{z}$-axis.

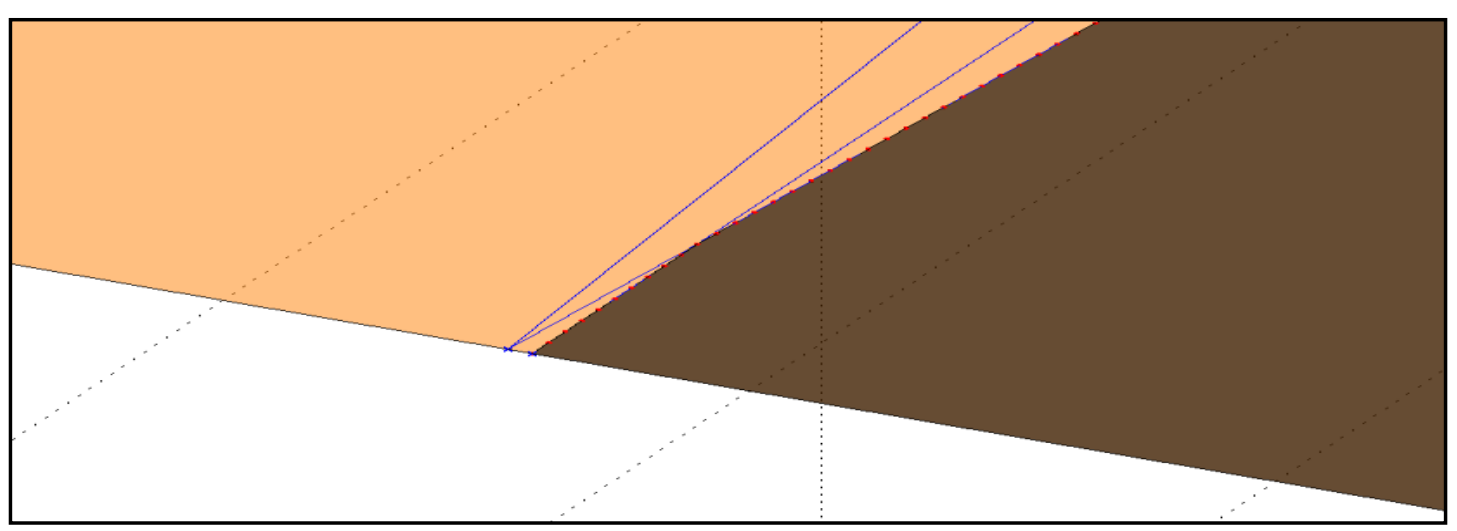

Fig. 21: Close-up of slicing 


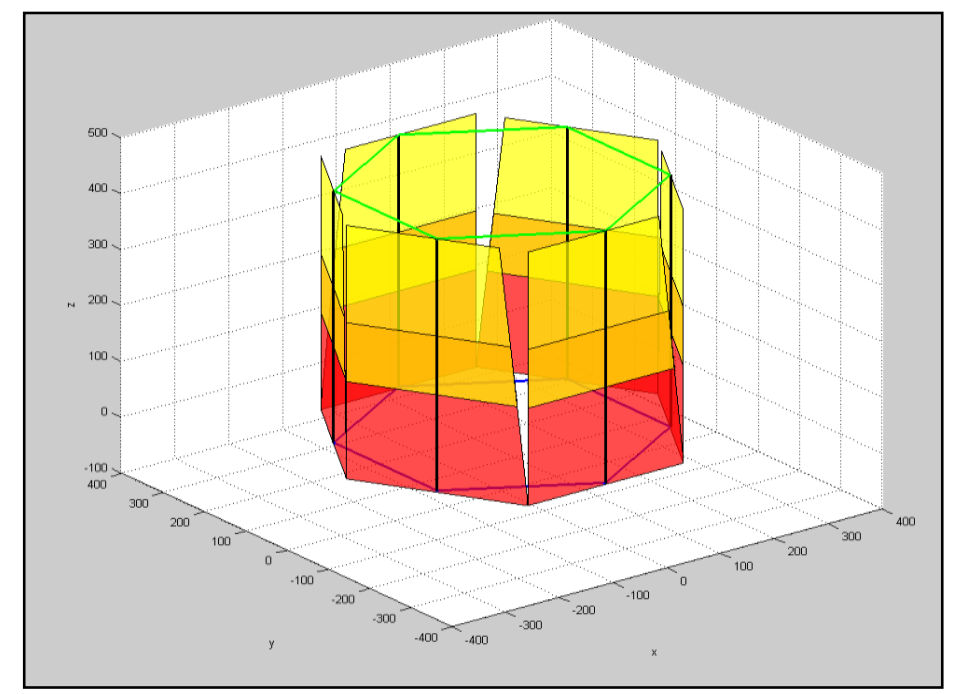

Fig. 22: Hexapod with sliced active panels

After slicing, the panels are re-illustrated with the hexapod to inspect the final design. Using the previous example, Fig. 22 displays the final active panel shape, with telescopic overlay included. The first thing noticed is the void formation from the slicing when the hexapod is subjected to global z-axial rotations. As mentioned previously, the gaps will be covered by flexible material or by a second layer of rigid skin panel. The approach of using flexible material is accomplished to reduce the amount of flexible material used while increasing the amount of rigid material. Unfortunately, two major problems can be seen with this approach. First, the active panel slicing will not allow for flush or streamline geometric shape. This is due to the telescopic mechanism that is required for six DOF morphing. For example in Fig. 22, it is obvious the surface area of the bottom panel is greater than the top panel. If span reduction morphing were to take place, the bottom panel will protrude the top panels from the side, causing sharp edges and vice versa. This leads to the second problem: the proper fitting of flexible material between panels. There is no optimal position to fit the flexible material to avoid the shearing that is caused during different morphing configurations. As a result, although active panel may not seem viable, it is a baseline research to aid the development of a new method, known as passive panel. 


\section{CHAPTER 5 Kinematic MODELling OF A}

\section{Passive Panel}

In this chapter, a second methodology is presented with the same hexapod and sliding skin mechanism. However, contrary to the first methodology, the skin panels are not attached directly to the active links. Instead, the sliding panels are extended off a joint (joints with two to three degrees of freedom) for additional DOF motion. The objective of this configuration is to have skin panels orientated passively based on the morphing mechanisms workspace. Theoretically, with the addition of the extra DOF, the reorientation of the panels should cause no collision in comparison to panels that are actively attached to the mechanism. For the purpose of this thesis, this will be referred as the passive panel. The jointed links may vary in design; the current prototype employs a skin panel that is rigidly attached to an extended S-joint. The Sjoints are connected at the base or the platform of the mechanism. A top view of the structure may be seen below:

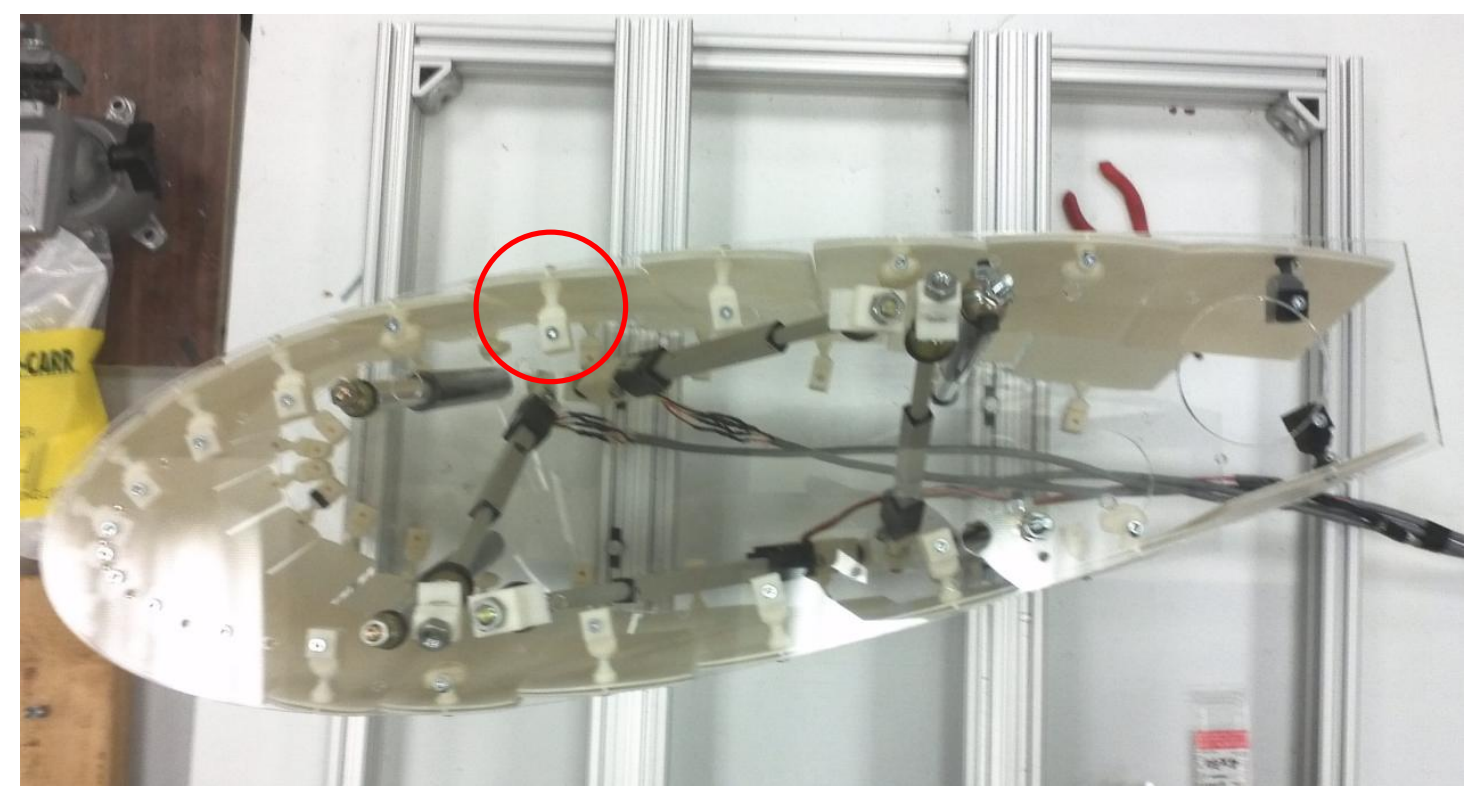

Fig. 23: Extended link of sliding panels adopted from [64] 
As seen in Fig. 23, the link is connected S-joint first at the airfoil and then rigidly attached to the sliding panel. This allow for more leniency for self-orientation under the influence of motion. The S-joint coupled with the extra length will allow for larger panel movement. To better visualize the design, a schematic can be seen below:

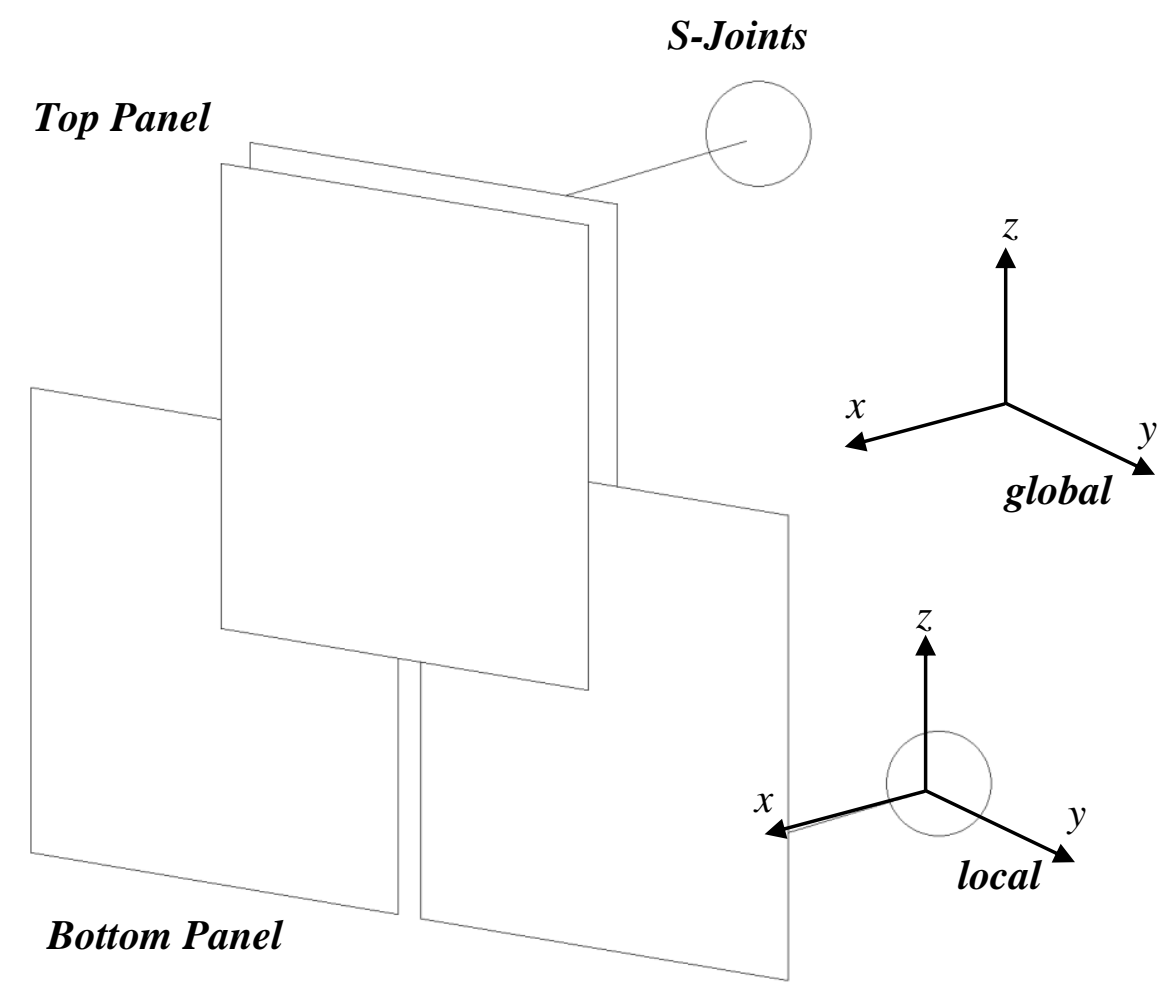

Fig. 24: Schematic of sliding panel prototype

For a single top panel, there will be two bottom panels connected to it. This setup is repeated until the entire mechanism is enclosed. As mentioned previously, the telescopic mechanism is incorporated in the design to allow for global z-axial translational morphing. This is achieved by sandwiching a double-layered top panel with a single layer bottom panel. There is a divider in the center of the top panel to hold the two layers together and separate the bottom panels from overlapping or interacting with each other. From this, it is understandable that if no extra links were added and the panels were directly attached to the links as an active panel, all global rotations will be restricted by the geometrical constraints of the system. Therefore, the 
extra links and S-joint are added to provide more allowable motion. However, S-joints appear to be redundant and it allows for local y-axial rotations, which is unnecessary since it will cause both the top and bottom panels to droop. In addition, the current configuration allows for twisting motion but requires the bending of panels. As seen in Fig. 23, panels near the upper and lower camber are misaligned, forming visible steps. Given that this sliding panel design is a mutually sandwiched structure among adjacent panels and bottom panels, misalignment while compromising with the morphing orientation indicates bending in the panels. Therefore, for the numerical analysis, the S-joint is replaced with an R-joint and instead of rigidly attaching the link to the panels, there will be an S-joint attachment purely for optimization of the zaxial rotation. Local $\mathrm{x}$ and $\mathrm{y}$-axial rotations are passively determined by the system, in other words it is impossible alter the local rotations without influencing the orientation of the internal morphing structure. Therefore, local $\mathrm{x}$ and $\mathrm{y}$-axial rotational angles are not considered for numerical analysis. A top view of the top panel schematic can be seen below. The bottom panel will follow the same configuration:

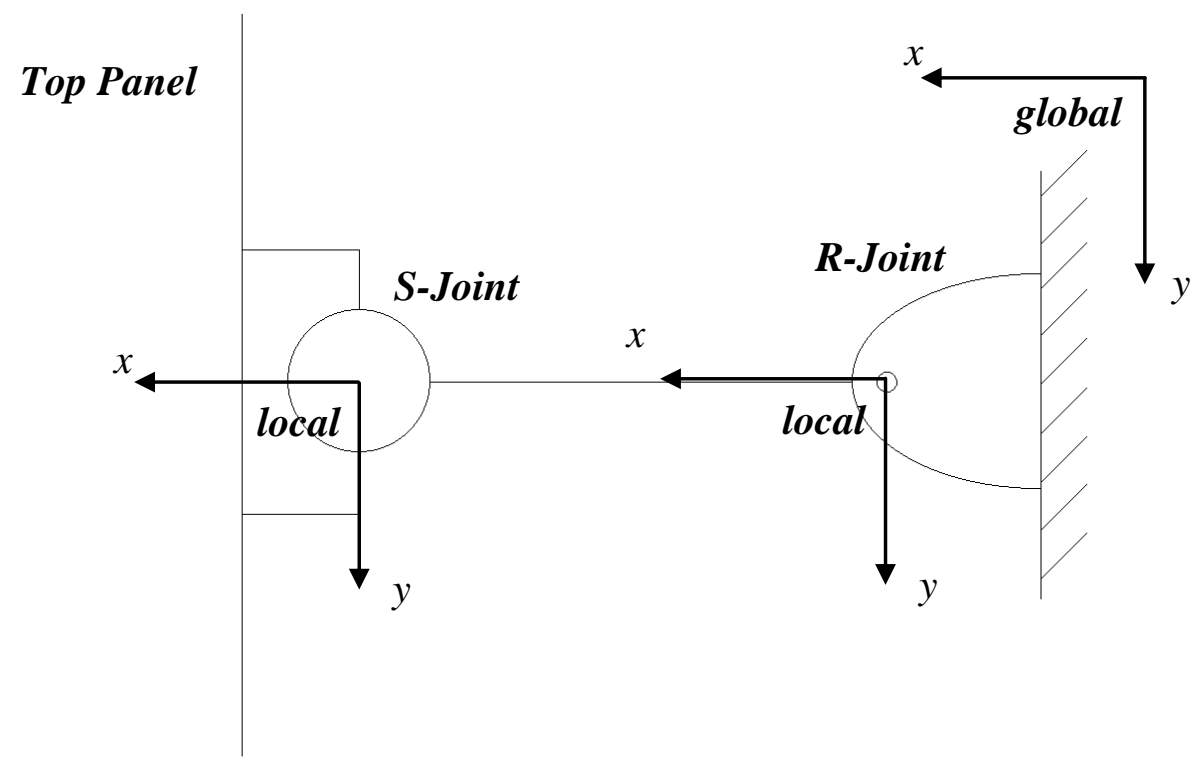

Fig. 25: Schematic of extra link for analysis 
The R-joint in combination with the local z-axial rotation of the S-joint will preserve the desired local z-axial rotation required for mechanism twist. The remaining $\mathrm{x}$ and $\mathrm{y}$-axial rotations of the $\mathrm{S}$-joint will preserve the twisting caused by global $\mathrm{y}$ and $\mathrm{x}$ axial translations, respectively. With the reposition of S-joint and addition of R-joint the sliding panels will provide better reorientation under global twisting conditions and limit the system from drooping. Although this does not fully resolve the bending of panels, it will provide some leniency when twisting.

\subsection{Passive Panel Modeling}

For the passive panel modeling, the telescopic mechanism will not sandwich two bottom panels. Instead it will sandwich only one. This is to simplify the system for validation of the feasibility for adding an extra link, and to obtain numerical formulation of panel interactions under passive conditions. This may seem inaccurate for only six panels. However when single telescopic sliding panel elements are increased, the formulated system will closely simulate panel interactions the sandwich prototype will have. The formulation of the passive panel will serve as a baseline analysis for the future works.

The ideal solution to the passive panel problem is to use the hexapod's workspace to calculate the angles of the R and S-joints, which will achieve said orientation without collision. The formulation of passive panels will follow the schematic diagram below: 


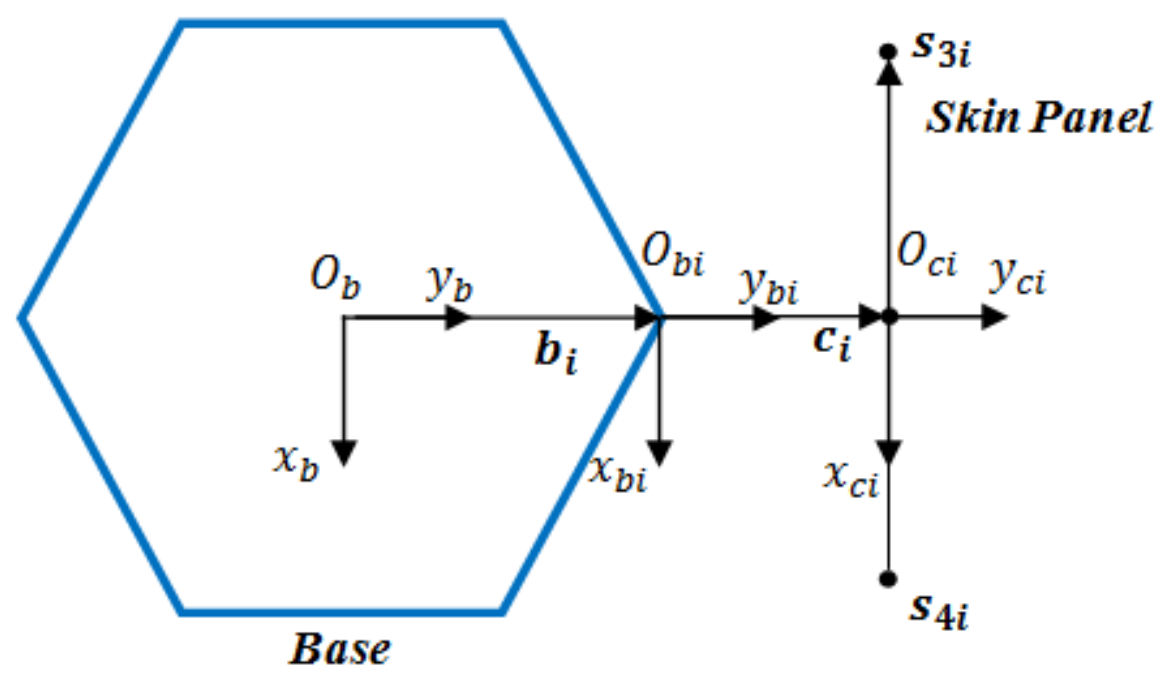

(a)

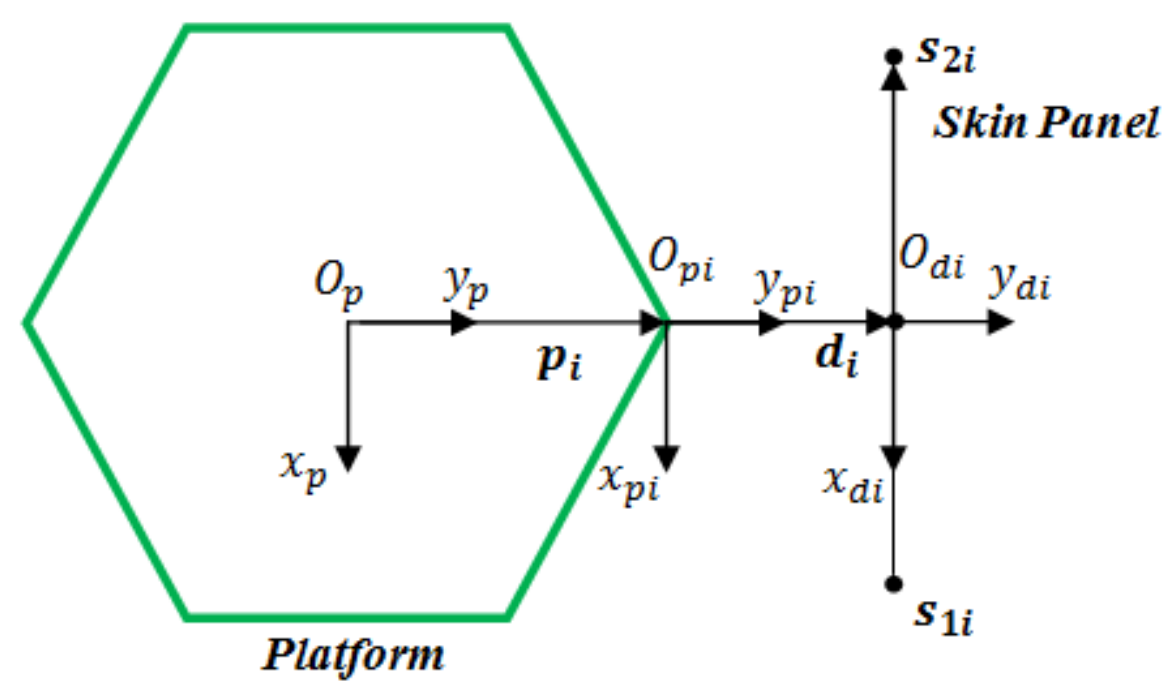

(b)

Fig. 26: Definition of passive panel configuration for base (a) and platform (b)

Using similar designations as the active panel hexapod configuration, extra links $\boldsymbol{c}_{i}$ and $\boldsymbol{d}_{i}$ for the base and platform were added respectively. The global basefixed frame $O_{b}-x_{b} y_{b} z_{b}$ and local platform-fixed frame $O_{p}-x_{p} y_{p} z_{p}$ remains the same. Local frames for the base and platform joints were added, $O_{b_{i}}-x_{b_{i}} y_{b_{i}} z_{b_{i}}$ is the 
local frame fixed to the respective $i$-th R-joint of the hexapod after base vector $\boldsymbol{b}_{i}$ and $O_{p_{i}}-x_{p_{i}} y_{p_{i}} z_{p_{i}}$ for the platform after platform vector $\boldsymbol{p}_{i}$. The co-ordinate frame is defined to be tangential to the circumference of the hexagonal geometry for each $i$-th branch. Similarly, $O_{c_{i}}-x_{c_{i}} y_{c_{i}} z_{c_{i}}$ is the local frame fixed to the respective $i$-th $S$-joint of the hexapod after link $\boldsymbol{c}_{i}$, and $O_{d_{i}}-x_{d_{i}} y_{d_{i}} z_{d_{i}}$ for the platform after link $\boldsymbol{d}_{i}$. Like the active panel configuration, each point vector $\left(\boldsymbol{s}_{j i}\right)$ for the base $\left(\boldsymbol{s}_{3 i}, \boldsymbol{s}_{4 i}\right)$ and platform $\left(\boldsymbol{s}_{1 i}, \boldsymbol{s}_{2 i}\right)$ can be obtained from the general equation of motion for multiple bodies, where ${ }_{f} \boldsymbol{q}_{k}$ is the position vector of each joint, where $f$ is the frame (i.e. base (b) or platform $(p))$, and $k$ is a position designation:

$$
\begin{aligned}
& \text { Base: } \quad j=3,4 \\
& \text { R-Joint }{ }_{b} \boldsymbol{q}_{1}=\boldsymbol{b}_{i} \\
& \text { S-Joint } \quad{ }_{b} \boldsymbol{q}_{2}={ }_{b} \boldsymbol{q}_{1}+\boldsymbol{R}_{b_{i}} \boldsymbol{c}_{i} \\
& \begin{array}{l}
\text { Final point } \quad{ }_{b} \boldsymbol{q}_{3}=\boldsymbol{s}_{j i}={ }_{b} \boldsymbol{q}_{2}+\boldsymbol{R}_{b_{i}} \boldsymbol{R}_{c_{i}} \boldsymbol{s}_{j i}^{\prime}
\end{array} \\
& \text { Platform: } \quad j=1,2 \\
& \text { R-Joint } \quad{ }_{p} \boldsymbol{q}_{1}=\boldsymbol{h}+\boldsymbol{R}_{p} \boldsymbol{b}_{i} \\
& \text { S-Joint } \quad{ }_{p} \boldsymbol{q}_{2}={ }_{p} \boldsymbol{q}_{1}+\boldsymbol{R}_{p_{i}} \boldsymbol{d}_{i}
\end{aligned}
$$

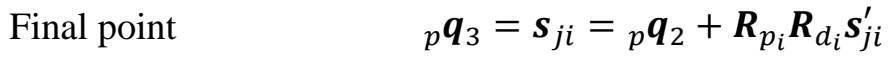

In Eq. (18), rotational matrix of the platform $\left(\boldsymbol{R}_{p}\right)$ is normally included in the equation of motions for ${ }_{p} \boldsymbol{q}_{2}$ and ${ }_{p} \boldsymbol{q}_{3}$, but including it will cause the panel to deform against its 
rigid properties. Therefore to simulate a fully rigid panel, the rotational matrix is not included in the formulation of ${ }_{p} \boldsymbol{q}_{2}$ and ${ }_{p} \boldsymbol{q}_{3}$, but will be kept in ${ }_{p} \boldsymbol{q}_{1}$.

In Eq. (17) and (18) The rotational matrices $\boldsymbol{R}_{\boldsymbol{b}_{i}}, \boldsymbol{R}_{\boldsymbol{c}_{i}}, \boldsymbol{R}_{\boldsymbol{p}_{i}}, \boldsymbol{R}_{\boldsymbol{d}_{i}}$ are defined by the following, where corresponding vector position subscript $\boldsymbol{b}_{i}, \boldsymbol{c}_{i}, \boldsymbol{p}_{i}$, and $\boldsymbol{d}_{i}$ are used as designations:

$$
\boldsymbol{R}_{q_{i}}={ }_{f, j, d} \boldsymbol{R}_{i s} \boldsymbol{R}_{i}
$$

The dynamic $\left({ }_{d} \boldsymbol{R}_{i}\right)$ and static $\left.{ }_{s} \boldsymbol{R}_{i}\right)$ rotational matrices are required to transform local co-ordinates to their respective global frame. Where subscript $f, j, d, s$ represent frame of system (i.e. base $(b)$ or platform $(p))$, type of joint $(R$ or $S)$, dynamic, and static respectively. $f, j, d$ 䠉 is simply defined by the input angles of the given joint, similar to the platform rotational matrix in Eq. (2):

$$
f, j, d \boldsymbol{R}_{i}=\left[\boldsymbol{R}_{x}\left(f, j \theta_{i, x}\right) \boldsymbol{R}_{y}\left(f, j \theta_{i, y}\right) \boldsymbol{R}_{z}\left({ }_{f, j} \theta_{i, z}\right)\right]
$$

Where, ${ }_{f, j} \theta_{i, a}$ is $(f)$ frame (i.e. base $(b)$ or platform $\left.(p)\right),(j)$ joint, $(i) i$-th branch, and (a) corresponding axis respectively. Each respective rotational matrix is defined:

$$
\begin{aligned}
& \boldsymbol{R}_{b_{i}}={ }_{b, R, d} \boldsymbol{R}_{i s} \boldsymbol{R}_{\boldsymbol{i}} \\
& \boldsymbol{R}_{c_{i}}={ }_{b, S, d} \boldsymbol{R}_{i s} \boldsymbol{R}_{\boldsymbol{i}} \\
& \boldsymbol{R}_{p_{i}}={ }_{p, R, d} \boldsymbol{R}_{i s} \boldsymbol{R}_{i} \\
& \boldsymbol{R}_{d_{i}}={ }_{p, S, d} \boldsymbol{R}_{i s} \boldsymbol{R}_{i}
\end{aligned}
$$

Given that an R-joint and S-joint, only $\theta_{z}$ of the base and platform, R-joint is required. As mentioned previously, due to the design of the system, $\theta_{x}$ and $\theta_{y}$ of the S-joint are restricted by the physical structure of the system. Local $x$ and $y$-axial rotations of the 
$\mathrm{S}$-joint are present to allow the skin panels motions, while the internal mechanism is under $\mathrm{y}$ and $\mathrm{x}$-axial translations, respectively. Modifying $\theta_{x}$ and $\theta_{y}$ will require the reorientation of the internal mechanism, which is not desired, since $\theta_{x}$ and $\theta_{y}$ are dependent on the orientation of the internal mechanism. Also in the simulation, modification of $\theta_{x}$ and $\theta_{y}$ for S-joints will not cause any changes to the skin panel or the orientation of the internal mechanism. Therefore, the only independent variable is $\theta_{z}$ of the S-joint, where $\theta_{x}$ and $\theta_{y}$ are set as zero. In addition, given that the S-joints share the same panel, the definition of one S-joint will be sufficient. Accordingly, the final unknowns of the problems are: ${ }_{b, R} \theta_{i, z}$ ( $\mathrm{z}$ angle of the base R-joint), ${ }_{p, R} \theta_{i, z}(\mathrm{z}$ angle of the platform $\mathrm{R}$-joint), and ${ }_{S} \theta_{i, z}$ (z angle of the S-joint). Where $i=1 \ldots 6$, totaling of eighteen variables.

The static rotational matrix is defined with respect to the defined local and global fixed frames. Since all local fixed frames are defined in a similar form, tangential to the circumference of the hexagonal geometry, the following is used to define each static rotational matrix for the respective $i$-th branch:

$$
{ }_{s} \boldsymbol{R}_{i}=\left[\begin{array}{ccc}
0 & \cos \theta_{\text {hex }} & \sin \theta_{\text {hex }} \\
0 & \sin \theta_{\text {hex }} & -\cos \theta_{\text {hex }} \\
1 & 0 & 0
\end{array}\right]_{\boldsymbol{i}}
$$

From Eq.(25), $\theta_{\text {hex }}$ is the current hexagonal angle at the $i$-th branch, ranging from 0 to 300 [deg] by $60[\mathrm{deg}]$ intervals. $\boldsymbol{s}_{j i}^{\prime}$ is the local co-ordinates of the skin panel, however definition of this value is not the same as the active panel configuration. In the case of the passive panel, $\boldsymbol{s}_{j i}$ is first evaluated with all joint angles predefined as zeroes. Once $\boldsymbol{s}_{j i}$ is obtained, $\boldsymbol{s}_{j i}^{\prime}$ is evaluated with either Eq. (17) or (18) for their respective frame. 


\subsection{Panel Orientation Criteria}

The main objective of the passive panel approach is to accommodate any form of hexapod orientation without the collision among adjacent sliding panels. As a result, the removal of excess material is not required, also the skin design complexity may be reduce and hence developing a morphing skin that may be used for a more general cases. In order to better position all sliding panels without collision and still enclose the internal components, the follow three criteria were developed.

1. Given two skin panels, panel 1 and panel 2, adjacent to each other. The spanwise right edge of panel 1 must be parallel with the span-wise left edge of panel 2.

2. The distance between the right edge of panel 1 and left edge of panel 2 must be close-to or equal-to zero.

3. The first two criteria must be valid for six skin panels all adjacent to each other. Panel 6 and panel 1 will be the end of a closed loop system.

Parallelism is desired for it is the most ideal position a panel can orientate into without colliding or intersecting another panel. As a result, a mutually dependant system is formed. The adjustment of one panel will unavoidably reorientate the remaining five panels, overall affecting the first affected panel in a continuous loop. Therefore, the three criteria are transformed into numerical representation to be evaluated simultaneously for a solution that satisfies all three conditions. The criteria are as follows, for the $i$-th branch of the hexapod:

$$
\begin{aligned}
& m_{x, i, \text { right }}=m_{x, i+1, \text { left }} \\
& m_{y, i, \text { right }}=m_{y, i+1, \text { left }}
\end{aligned}
$$




$$
d_{i, r i g h t}=d_{i+1, l e f t}
$$

To be able to program the criteria, they are transformed to equate numerical values:

$$
\begin{gathered}
m_{x, i, r i g h t}-m_{x, i+1, \text { left }}=m_{x, i, L R}=0 \\
m_{y, i, r i g h t}-m_{y, i+1, \text { left }}=m_{y, i, L R}=0 \\
d_{i, \text { right }}-d_{i+1, \text { left }}=d_{i, L R}=0
\end{gathered}
$$

From Eq.(26) and (27), $m_{x}$ and $m_{y}$ are the $\mathrm{x}$ and $\mathrm{y}$ components of the directional vector $\left(\boldsymbol{m}=\left[m_{x} m_{y} m_{z}\right]^{\boldsymbol{T}}\right)$ between the span-wise co-ordinates of the skin panel. The directional vector $\boldsymbol{m}$ is defined as:

$$
\begin{gathered}
\boldsymbol{m}_{i, \text { right }}=\frac{\boldsymbol{s}_{2 i}-\boldsymbol{s}_{3 i}}{\left\|\boldsymbol{s}_{2 i}-\boldsymbol{s}_{3 i}\right\|} \\
\boldsymbol{m}_{i, \text { left }}=\frac{\boldsymbol{s}_{1 i}-\boldsymbol{s}_{4 i}}{\left\|\boldsymbol{s}_{1 i}-\boldsymbol{s}_{4 i}\right\|}
\end{gathered}
$$

From Eq.(28), $d$ is the scalar distance between the left and right edges of the $i$-th hexagon branch. The method used is the shortest distance between point to line formulation, defined as:

$$
\begin{gathered}
d_{i, \text { right }}=\left\|\boldsymbol{s}_{\mathbf{2 i}}-\left(\boldsymbol{s}_{\mathbf{2 i}} \cdot m_{i, \text { right }}\right) m_{i, \text { right }}\right\| \\
d_{i, \text { left }}=\left\|\boldsymbol{s}_{\mathbf{1 i}}-\left(\boldsymbol{s}_{\mathbf{1 i}} \cdot m_{i, \text { left }}\right) m_{i, \text { left }}\right\|
\end{gathered}
$$

When any of the three criteria is equated to the 6-th branch, the relations for the criteria will be equated between the 6-th and 1-st branch instead of $i+1$ branch. The angles of interest is the local z-axial rotation of the two R-joints $\left({ }_{b, R} \theta_{i, z}, p, R=\theta_{i, z}\right)$, and the local z-axial rotation of the S-joint $\left({ }_{S} \theta_{i, z}\right)$. Since the $S$-joints are coplanar, only 
one S-joint angle is required for each panel. As a result, there will be eighteen unknowns and eighteen equations. The eighteen available equations are as follows:

$$
\begin{array}{cccccc}
m_{x, 1, L R}=0 & m_{x, 2, L R}=0 & m_{x, 3, L R}=0 & m_{x, 4, L R}=0 & m_{x, 5, L R}=0 & m_{x, 6, L R}=0 \\
m_{y, 1, L R}=0 & m_{y, 2, L R}=0 & m_{y, 3, L R}=0 & m_{y, 4, L R}=0 & m_{y, 5, L R}=0 & m_{y, 6, L R}=0 \\
d_{1, L R}=0 & d_{2, L R}=0 & d_{3, L R}=0 & d_{4, L R}=0 & d_{5, L R}=0 & d_{6, L R}=0
\end{array}
$$

The corresponding eighteen variables:

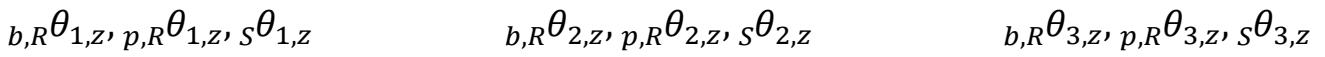

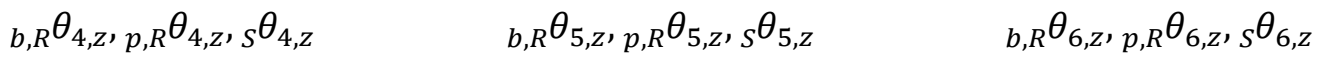

To understand the complexity of the system, a sample is formulated for panels with 346.42 units in width. The hexapod orientation is translated along global z-axis by 350 units with no other orientations. The following equations are obtained for a single set of criteria between panels on branch 1 and branch 2: 
$m_{x, 1, L R}=\frac{20 \sin \left({ }_{b, R} \theta_{1, z}\right)-20 \sin \left(_{p, R} \theta_{1, z}\right)}{\sqrt{\left|20 \sin \left({ }_{b, R} \theta_{1, z}\right)-20 \sin \left({ }_{p, R} \theta_{1, z}\right)\right|^{2}+\left|20 \cos \left({ }_{b, R} \theta_{1, Z}\right)-20 \cos \left(_{p, R} \theta_{1, z}\right)\right|^{2}+1.22 E 5}}$

$10 \sin \left({ }_{b, R} \theta_{2, z}\right)-10 \sin \left({ }_{p, R} \theta_{2, z}\right)+17.3 \cos \left({ }_{b, R} \theta_{2, z}\right)-17.3 \cos \left({ }_{p, R} \theta_{2, z}\right)$

$\sqrt{\left|10 \cos \left({ }_{b, R} \theta_{2, z}\right)-10 \cos \left({ }_{p, R} \theta_{2, z}\right)-17.3 \sin \left({ }_{b, R} \theta_{2, z}\right)+17.3 \sin \left({ }_{p, R} \theta_{2, z}\right)\right|^{2}+\left|10 \sin \left({ }_{b, R} \theta_{2, z}\right)-10 \sin \left({ }_{p, R} \theta_{2, z}\right)+17.3 \cos \left({ }_{b, R} \theta_{2, z}\right)-17.3 \cos \left({ }_{p, R} \theta_{2, z}\right)\right|^{2}+1.22 E 5}$

$m_{y, 1, L R}=\frac{10 \cos \left({ }_{b, R} \theta_{2, z}\right)-10 \cos \left({ }_{p, R} \theta_{2, z}\right)-17.3 \sin \left({ }_{b, R} \theta_{2, z}\right)+17.3 \sin \left({ }_{p, R} \theta_{2, z}\right)}{\sqrt{\left|17.3 \cos \left(_{b, R} \theta_{2, z}\right)-17.3 \cos \left(_{p, R} \theta_{2, z}\right)+10 \sin -10 \sin \left({ }_{p, R} \theta_{2, z}\right)\right|^{2}+\left|10 \cos \left(_{b, R} \theta_{2, z}\right)-10 \cos \left(_{p, R} \theta_{2, z}\right)-17.3 \sin \left({ }_{b, R} \theta_{2, z}\right)+17.3 \sin \left(_{p, R} \theta_{2, z}\right)\right|^{2}+1.22 E 5}}$ $20 \cos \left({ }_{b, R} \theta_{1, z}\right)-20 \cos \left({ }_{p, R} \theta_{1, z}\right)$

$\sqrt{\left|20 \sin \left({ }_{b, R} \theta_{1, z}\right)-20 \sin \left(_{p, R} \theta_{1, z}\right)\right|^{2}+\left|20 \cos \left({ }_{b, R} \theta_{1, z}\right)-20 \cos \left(_{p, R} \theta_{1, z}\right)\right|^{2}+1.22 E 5}$

$d_{1, L R}=\left(\left|173 \sin \left({ }_{s} \theta_{1, Z}+{ }_{b, R} \theta_{1, z}\right)-20 \cos \left({ }_{p, R} \theta_{1, z}\right)+10 \cos \left({ }_{p, R} \theta_{2, z}\right)-17.3 \sin \left({ }_{p, R} \theta_{2, z}\right)+\cos \left({ }_{s} \theta_{2, z}\right) A_{4}-\cos \left({ }_{s} \theta_{2, z}\right) A_{1}+\sin \left({ }_{s} \theta_{2, z}\right)+\sin \left({ }_{s} \theta_{2, z}\right) A_{3}+\frac{A_{15} A_{8}}{A_{11}}-\frac{A_{7} A_{13}}{A_{9}}-125\right|^{2}\right.$

$+\mid\left(\left(8.75 E 16 \cos \left({ }_{p, R} \bar{\theta}_{1, z}-{ }_{p, R} \theta_{1, z}\right)-8.75 E 16 \cos \left({ }_{p, R} \bar{\theta}_{1, z}-{ }_{b, R} \theta_{1, z}\right)-1.09 E 18 \cos \left({ }_{b, R} \theta_{1, z}\right)+1.09 E 18 \cos \left({ }_{p, R} \theta_{1, z}\right)+67 \sin \left({ }_{b, R} \theta_{1, z}\right)-67 \sin \left(p, R \theta_{1, z}\right)\right.\right.$

$\left.\left.+7.57 E 17 \sin \left({ }_{s} \bar{\theta}_{1, z}-{ }_{b, R} \theta_{1, z}-{ }_{b, R} \bar{\theta}_{1, z}\right)-7.57 E 17 \sin \left({ }_{s} \bar{\theta}_{1, z}-{ }_{p, R} \theta_{1, z}+{ }_{b, R} \bar{\theta}_{1, z}\right)\right) /\left(6.25 E 11 A_{15}^{2}+6.25 E 11 A_{16}^{2}+7.66 E 16\right)\right)-\left(\left(7 E 5 \cos \left({ }_{p, R} \bar{\theta}_{2, z}-{ }_{p, R} \theta_{2, z}\right)\right.\right.$

$\left.-7 E 5 \cos \left({ }_{p, R} \bar{\theta}_{2, z}-{ }_{b, R} \theta_{1, z}\right)-8.75 E 6 \cos \left({ }_{b, R} \theta_{2, z}\right)+8.75 E 6 \cos \left({ }_{p, R} \theta_{2, z}\right)-154 \cos \left(A_{6}\right)+154 \cos \left(A_{5}\right)-6.06 E 6 \sin \left(A_{6}\right)+6.06 E 6 \sin \left(A_{5}\right)+2.14 E 8\right) /\left(5 A_{14}^{2}+5 A_{13}^{2}\right.$ $+6.12 E 5))\left.\right|^{2}$

$+\mid-173 \cos \left({ }_{s} \theta_{1, z}+{ }_{b, R} \theta_{1, z}\right)+17.3 \cos \left({ }_{p, R} \theta_{2, z}\right)-20 \sin \left({ }_{p, R} \theta_{1, z}\right)+10 \sin \left({ }_{p, R} \theta_{2, z}\right)+\sin \left({ }_{s} \theta_{2, z}\right) A_{4}-\cos \left({ }_{s} \theta_{2, z}\right) A_{2}+\sin \left({ }_{s} \theta_{2, z}\right) A_{1}-\cos \left({ }_{s} \theta_{2, z}\right) A_{3}+\frac{A_{15} A_{8}}{A_{11}}-\frac{A_{7} A_{14}}{A_{9}}$ $\left.+\left.216\right|^{2}\right)^{\frac{1}{2}}$

Where, 


$$
A_{3}=86.6 \cos \left({ }_{b, R} \theta_{2, z}\right)-150 \sin \left({ }_{b, R} \theta_{2, z}\right)
$$

$$
A_{5}={ }_{s} \bar{\theta}_{2, z}-{ }_{p, R} \theta_{2, z}+{ }_{b, R} \bar{\theta}_{2, z}
$$

$A_{4}=150 \cos \left({ }_{b, R} \theta_{2, z}\right)+86.6 \sin \left({ }_{b, R} \theta_{2, z}\right)$

$$
A_{6}={ }_{s} \bar{\theta}_{2, z}-{ }_{b, R} \theta_{2, z}+{ }_{b, R} \bar{\theta}_{2, z}
$$

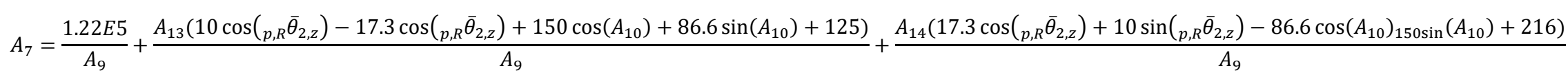

$$
A_{8}=-\frac{1.22 E 5}{A_{11}}+\frac{A_{16}\left(20 \cos \left({ }_{p, R} \bar{\theta}_{1, z}\right)-173 \sin \left(A_{12}\right)+250\right)}{A_{11}}+\frac{A_{15}\left(20 \sin \left({ }_{p, R} \bar{\theta}_{1, z}\right)+173 \cos \left(A_{12}\right)-1.53 E-14\right)}{A_{11}}
$$

$$
A_{9}=\sqrt{\left|A_{14}\right|^{2}+\left|A_{13}\right|^{2}+1.22 E 5}
$$$$
A_{11}=\sqrt{\left|A_{15}\right|^{2}+\left|A_{16}\right|^{2}+1.22 E 5}
$$

$A_{13}=10 \cos \left({ }_{b, R} \theta_{2, z}\right)-10 \cos \left({ }_{p, R} \theta_{2, z}\right)-17.3 \sin \left({ }_{b, R} \theta_{2, z}\right)+17.3 \sin \left({ }_{p, R} \theta_{2, z}\right)$

$A_{15}=20 \sin \left({ }_{b, R} \theta_{1, z}\right)-20 \sin \left({ }_{p, R} \theta_{1, z}\right)$

$$
\begin{aligned}
& A_{10}={ }_{s} \bar{\theta}_{2, z}+{ }_{b, R} \bar{\theta}_{2, z} \\
& A_{12}={ }_{s} \bar{\theta}_{1, z}+{ }_{b, R} \bar{\theta}_{1, z}
\end{aligned}
$$

$A_{14}=17.3 \cos \left({ }_{b, R} \theta_{2, z}\right)-17.3 \cos \left({ }_{p, R} \theta_{2, z}\right)+10 \sin \left({ }_{b, R} \theta_{2, z}\right)-10 \sin \left({ }_{p, R} \theta_{2, z}\right)$

$A_{16}=20 \cos \left({ }_{b, R} \theta_{1, z}\right)-\cos \left({ }_{p, R} \theta_{1, Z}\right)$ 


\subsection{Linear Formulation: Newton-Raphson for Panel Orientation OPTIMIZATION}

The three criteria are derived with these two baseline equations (Eq. (17) and (18)) and can be characterized as a series of nonlinear trigonometric functions. Given the highly complex system, a direct evaluation of the solution may be a resource and time consuming process and not within the scope of this thesis. Therefore, linear approximation methods such as the Newton-Raphson method are adopted to calculate the angles that satisfy the three criteria. The following Newton-Raphson method for nonlinear system of equations is used:

$$
\begin{gathered}
F(x) \approx F\left(x_{o}\right)+\boldsymbol{J}\left(x-x_{o}\right) \\
x=x_{o}+\boldsymbol{J}^{-1}\left[F(x)-F\left(x_{o}\right)\right]
\end{gathered}
$$

Eq. (36) is the Newton-Raphson equation for solving nonlinear systems; this can be rearranged to solve for $x$, in this case the angles of the morphing skin. Only the first order of the Jacobian is considered for simplicity and faster approximations when computing. For Eq.(37), $F(x)$ is evaluated as zero to match with the three criteria. The function $F\left(x_{o}\right)$ will be the eighteen equations evaluated at the initial arbitrary values defined by the user. The Jacobian matrix $(\boldsymbol{J})$ is a matrix of all first-order partial derivatives of the eighteen functions. Therefore, the Jacobian matrix for this case will be an $18 \times 18$ matrix (Eq. (38)). While executing the Newton-Raphson method on MATLAB, three issues arose. First obvious issue that will render this method obsolete is the singularity caused by the inverse of the Jacobian matrix. Fortunately, for asymmetrical hexapod orientations, such as global $\mathrm{x}$-axial rotational twist with y-axial 
translation, the Jacobian matrix is invertible. But this is only because the directional vectors are non zero.

$$
\boldsymbol{J}=\left[\begin{array}{lllllll}
\frac{\delta m_{x, 1, L R}}{\delta_{b, R} \theta_{1, z}} & \frac{\delta m_{x, 1, L R}}{\delta_{p, R} \theta_{1, z}} & \frac{\delta m_{x, 1, L R}}{\delta_{S} \theta_{1, z}} & & \frac{\delta m_{x, 1, L R}}{\delta_{b, R} \theta_{6, z}} & \frac{\delta m_{x, 1, L R}}{\delta_{p, R} \theta_{6, z}} & \frac{\delta m_{x, 1, L R}}{\delta_{S} \theta_{6, z}} \\
\frac{\delta m_{y, 1, L R}}{\delta_{b, R} \theta_{1, z}} & \frac{\delta m_{y, 1, L R}}{\delta_{p, R} \theta_{1, z}} & \frac{\delta m_{y, 1, L R}}{\delta_{S} \theta_{1, z}} & \cdots & \frac{\delta m_{y, 1, L R}}{\delta_{b, R} \theta_{6, z}} & \frac{\delta m_{y, 1, L R}}{\delta_{p, R} \theta_{6, z}} & \frac{\delta m_{y, 1, L R}}{\delta_{S} \theta_{6, z}} \\
\frac{\delta d_{1, L R}}{\delta_{b, R} \theta_{1, z}} & \frac{\delta d_{1, L R}}{\delta_{p, R} \theta_{1, z}} & \frac{\delta d_{1, L R}}{\delta_{S} \theta_{1, z}} & & \frac{\delta d_{1, L R}}{\delta_{b, R} \theta_{6, z}} & \frac{\delta d_{1, L R}}{\delta_{p, R} \theta_{6, z}} & \frac{\delta d_{1, L R}}{\delta_{S} \theta_{6, z}} \\
\frac{\delta m_{x, 6, L R}}{\delta_{b, R} \theta_{1, z}} & \frac{\delta m_{x, 6, L R}}{\delta_{p, R} \theta_{1, z}} & \frac{\delta m_{x, 6, L R}}{\delta_{S} \theta_{1, z}} & & \frac{\delta m_{x, 6, L R}}{\delta_{b, R} \theta_{6, z}} & \frac{\delta m_{x, 6, L R}}{\delta_{p, R} \theta_{6, z}} & \frac{\delta m_{x, 6, L R}}{\delta_{S} \theta_{6, z}} \\
\frac{\delta m_{y, 6, L R}}{\delta_{b, R} \theta_{1, z}} & \frac{\delta m_{y, 6, L R}}{\delta_{p, R} \theta_{1, z}} & \frac{\delta m_{y, 6, L R}}{\delta_{S} \theta_{1, z}} & \cdots & \frac{\delta m_{y, 6, L R}}{\delta_{b, R} \theta_{6, z}} & \frac{\delta m_{y, 6, L R}}{\delta_{p, R} \theta_{6, z}} & \frac{\delta m_{y, 6, L R}}{\delta_{S} \theta_{6, z}} \\
\frac{\delta d_{6, L R}}{\delta_{b, R} \theta_{1, z}} & \frac{\delta d_{6, L R}}{\delta_{p, R} \theta_{1, z}} & \frac{\delta d_{6, L R}}{\delta_{S} \theta_{1, z}} & & \frac{\delta d_{6, L R}}{\delta_{b, R} \theta_{6, z}} & \frac{\delta d_{6, L R}}{\delta_{p, R} \theta_{6, z}} & \frac{\delta d_{6, L R}}{\delta_{S} \theta_{6, z}}
\end{array}\right]
$$

However, a few cases where the hexapod has symmetrical orientations, such as pure global x-axial translation or even no morphing orientation, directional vectors are zero, resulting in the possibility of a degenerate matrix. Most importantly, the neutral configuration where all no orientations are present, singularity occurs. This makes it one of the leading concluding factors that this method is inconsistent and unreliable. Other two issues are minor compared to the singularity issue; they involve the lack of boundary limitation and increase in complexity in code. With the lack of boundary limitation, the iterated solution often results in numbers less than 0 [deg] and greater than 360 [deg]. However given that the equations are trigonometric, the iterated solutions can be readjusted to match a corresponding value that lies within the 0 to 360 [deg] threshold. Given that Jacobian matrix is a combination of partial derivatives, symbolic equations are required before substitution of actual values. As a result, derivation and processing of symbolic functions within MATLAB is a complex and time consuming task. Overall, even though Newton-Raphson method presents a rather 
promising solution to a complex problem, it is unfortunately obsolete due to its lack of robustness in the method. As a result, other alternatives are examined.

\subsection{Non-Linear Formulation: MUltiobJective OPTIMization FOR PANEL ORIENTATION OPTIMIZATION}

Since linear methods were not a reliable solution to the passive panel problem, nonlinear methods were investigated. With the recent update of MATLAB R2015a, an optimization toolbox has been published. Some notable popular optimization methods include the genetic algorithm (GA) and the sequential quadratic programming (SQP). GA and SQP were both tested in the process of examining alternate solving methods. In-depth explanation and analysis of optimization methods are out of the scope of this thesis. In brief, GA algorithm was performed with MATLAB function ga( ) and it is slow due to the properties of GA itself. GA will iterate population sizes defined by user, therefore with massive quantity of equations and variables computation time will increase. A 500 population size ran for maximum of 1000 iterations took almost 24 hours to run. On the other hand, SQP, performed with fmincon( ), took 10 minutes to run for the same preset conditions. Although the difference in efficiency is drastic, one major problem arose. The problem defined in this thesis is a multiobjective problem. Therefore, for the GA and SQP analysis, a single fitness function was not sufficient to define all criteria set in chapter 5.2, hence multiobjective optimization techniques were investigated.

From the optimization toolbox in MATLAB, there are not many options for multiobjective optimization. As MathWorks ${ }^{\circledR}$ stated themselves, they have three common multiobjective optimization: goal attainment, Minimax, and multiobjective genetic algorithm. Goal attainment reduces values of linear or nonlinear vector 
function to attain the goal values provided in the goal vector. The importance of the goal vector is defined by a weight vector. In addition, linear or nonlinear constraints may be applied. Minimax minimizes values of a multivariate function that may be subjected to linear or nonlinear constraints. Multiobjective genetic algorithm is as the name implies, GA with multiobjective definition [65].

The study of multiobjective optimization is no within the scope of this thesis, the purpose is to adopt existing optimization techniques to obtain results. MathWorks ${ }^{\circledR}$ documentations instructions will be followed to perform optimization. Therefore, debugging or improving will not be included in this thesis. In addition, if any method fails, it is due to lack of experience or documentation. Goal attainment is achieved with fgoalattain( ), Minimax is with fminimax( ), and finally multiobjective GA is with gamultiobj( ). The results will be discussed in the following chapter 6.2.

Given that goal attainment method does not require a constraint definition, objective functions will correspond to the eighteen developed criteria functions. Therefore, eighteen multiobjective functions will be minimized simultaneously. As for the remaining two methods, the distance criterion will be the objective function and parallelism criterion functions will be constraints. Therefore, six multiobjective functions will be solved for, with respect to twelve constraint functions. 


\section{CHAPTER 6 Passive Panel Simulation}

\subsection{Motion Analysis of Passive Skin Panels}

A general output of the passive panel modeling program can be seen below:

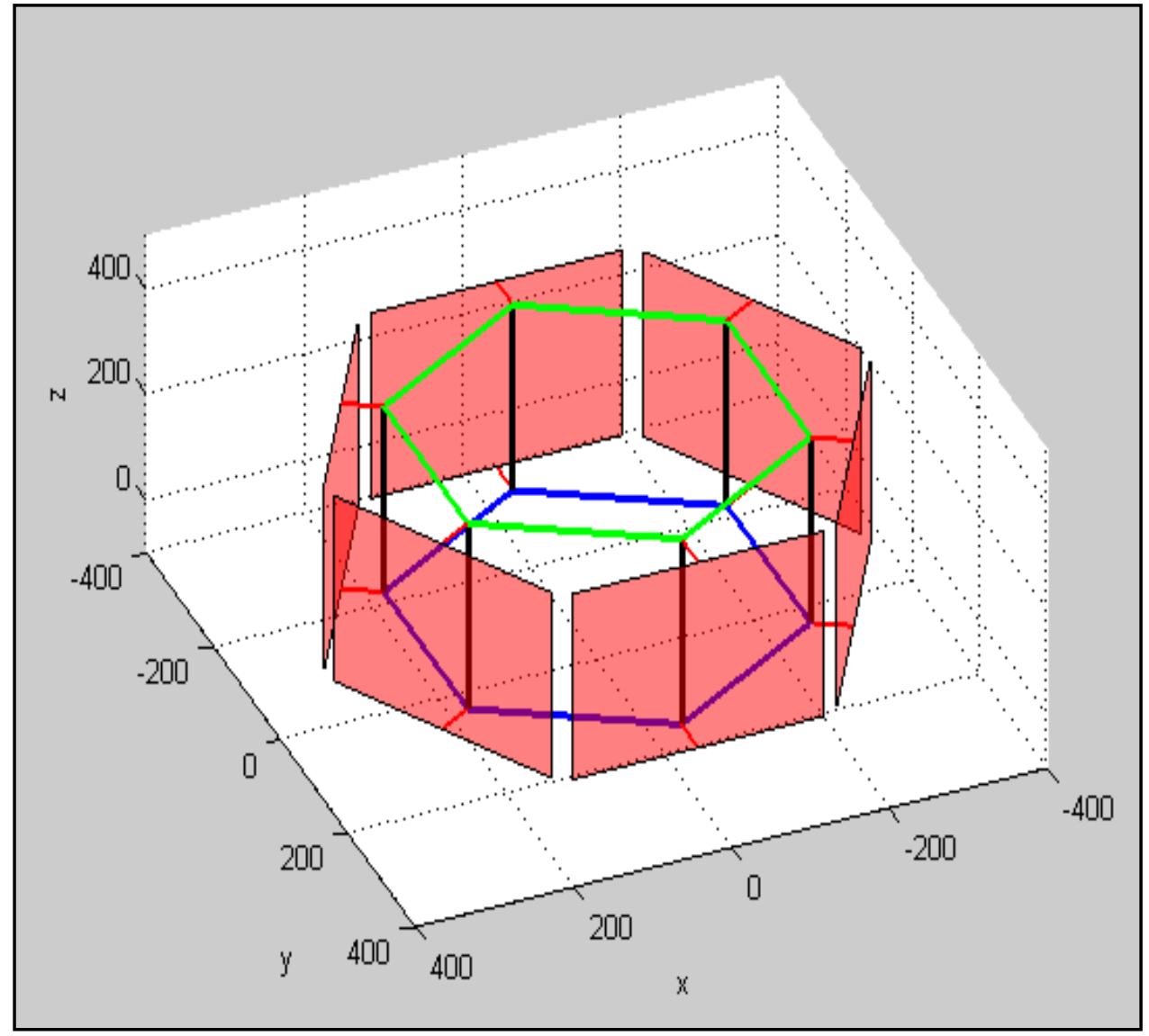

Fig. 27: Example of hexapod with passive skin panels. Baseline orientation

In Fig. 27, the hexapod orientation and all joint angles are all zero; this is defined as the baseline orientation. Skin panels and extra links are color coded in red. The platform and base uses the same color defined in the active panel modeling. Telescopic mechanism is not modelled for this optimization, because it will not affect the overall outcome of the solution. Therefore, a single skin panel that is capable of span morphing is modeled instead. The width of individual skin panels can be changed to minimize distance among adjacent panels, as seen in the figure, a visible 
gap is present among the six panels. Ideally, the gap will be much smaller than that, as seen in the prototype. For presentation, a larger gap is present to illustrate the capabilities of the program. This orientation is used to initialize all optimization methods to understand the changes and to investigate if the predefined functions can solve a simple system with the expected results. If repeated failures occur while attempting to debug the function, the said optimization function will be excluded as a solution option. The following table will be used for comparison purposes:

Table 1: Baseline orientation criteria results

\begin{tabular}{|c|c|c|c|c|c|c|}
\hline \multirow{3}{*}{\multicolumn{2}{|c|}{$\begin{array}{l}\text { Global x-axial twist: } 0[\mathrm{deg}] \\
\text { Global y-axial twist: } 0[\mathrm{deg}] \\
\text { Global z-axial twist: } 0[\mathrm{deg}]\end{array}$}} & & & & & \\
\hline & & \multicolumn{5}{|c|}{ Global y-axial translation: 0} \\
\hline & & \multicolumn{5}{|c|}{ Global z-axial translation: 350} \\
\hline Branch $i$ & 1 & 2 & 3 & 4 & 5 & 6 \\
\hline$m_{x, i, L R}$ & 0 & 0 & 0 & 0 & 0 & 0 \\
\hline$m_{y, i, L R}$ & 0 & 0 & 0 & 0 & 0 & 0 \\
\hline$d_{i, L R}$ & 25 & 25 & 25 & 25 & 25 & 25 \\
\hline
\end{tabular}

\subsection{Panel Orientation Optimization}

All three multiobjective optimization methods predefined by MATLAB were tested and it was concluded goal attainment method works the best. The Minimax method converged to a solution, however the results was not ideal, not all objectives were met. Specifically the directional vector criteria, all directional vectors were indifferent, but distance among panels was indeed minimized. In other words, adjacent panels collided among each other and the point of intersection provides the small distance between the panels.

\subsubsection{Goal Attainment}

Each MATLAB multiobjective optimization function was tested with two standard configurations: the baseline orientation (all global zero angles and translation) and 10 [deg] global z-axial twist. If the MATLAB function passes these two preliminary tests, 
it will then proceed to test other hexapod orientations. However, as mentioned previously, if the function repeatedly fails these two preliminary orientations, the said function will be discarded as a potential solution.

Goal attainment was very straightforward to program, the goal was to minimize the relations of the three criteria, all weighted equally. A 1000 iteration cycle usually converges in approximately a minute or less. In addition to the rapid computational time, the results were generated were also adequate. First, baseline orientation was achieved. The following optimization spread was obtained:

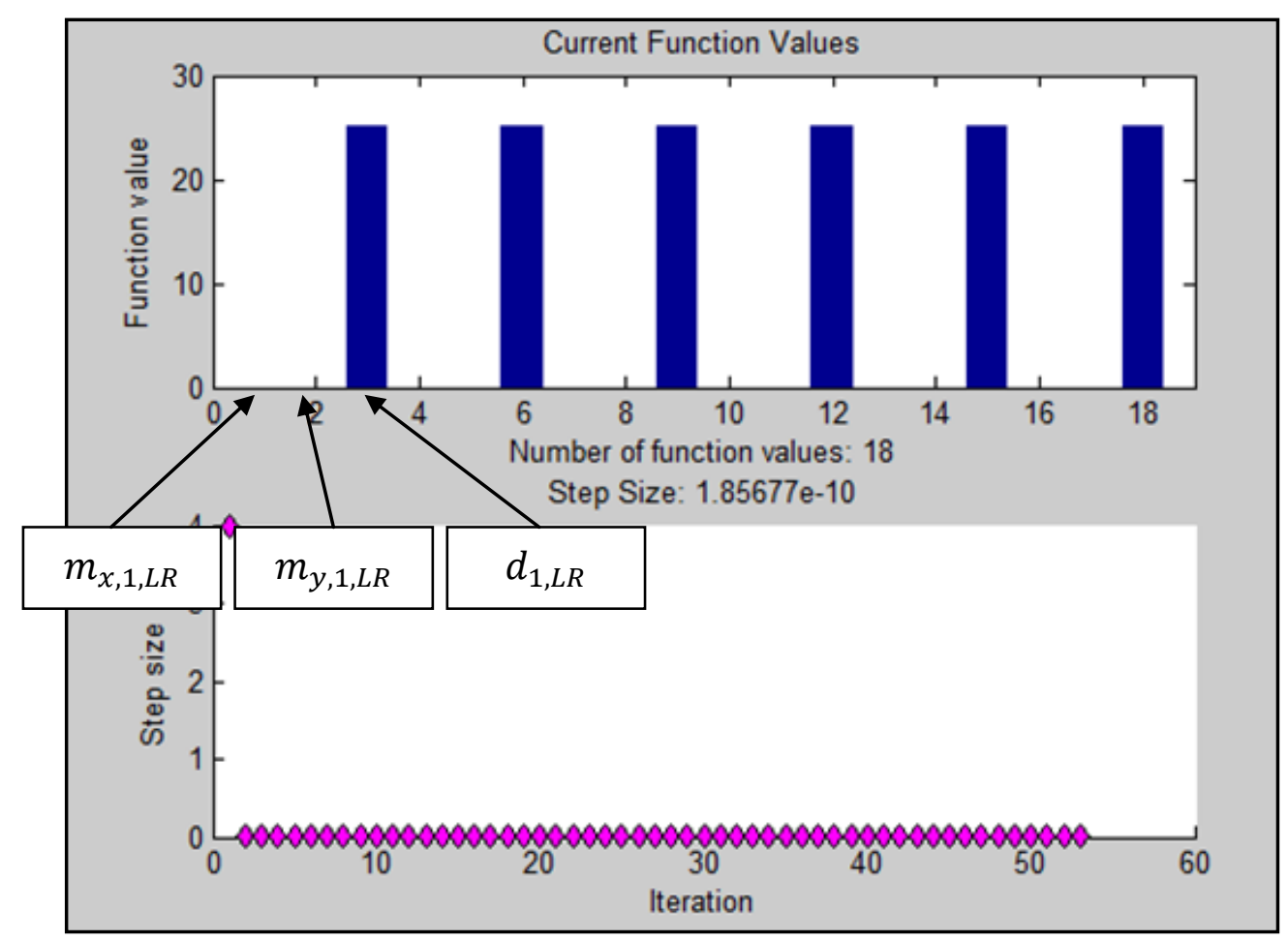

Fig. 28: Goal attainment for baseline orientation

Table 2: Goal attainment criteria results - baseline orientation

\begin{tabular}{|c|c|c|c|c|c|c|}
\hline $\begin{array}{l}\text { Global x-axial twist: } 0[\mathrm{deg}] \\
\text { Global y-axial twist: } 0[\mathrm{deg}] \\
\text { Global z-axial twist: } 0[\mathrm{deg}]\end{array}$ & $\begin{array}{l}\text { Global x-axial translation: } 0 \\
\text { Global y-axial translation: } 0\end{array}$ \\
\hline Branch $i$ & 1 & 2 & 3 & 4 & 5 & 6 \\
\hline$m_{x, i, L R}$ & 0 & 0 & 0 & 0 & 0 & 0 \\
\hline$m_{y, i, L R}$ & 0 & 0 & 0 & 0 & 0 & 0 \\
\hline$d_{i, L R}$ & 25 & 25 & 25 & 25 & 25 & 25 \\
\hline
\end{tabular}


From Fig. 28, the top graph is the function values of all the three criteria for six panels. The bottom graph is not as significant, it is solely for debugging. It displays the change in step size of iterated values and numbers of iteration, here it is seen that it converged rapidly. For the top graph, there are eighteen bars in total, but due to scaling, they may not be visible on the graph, since the directional vectors are all zero or close to zero. The visible bars are the distance criteria, all expected to equidistant among adjacent panels, as shown in Table 2. Given the match in results between Table 1 and Table 2, it implies goal attainment met the requirements for the baseline configuration. It is important to note that the iterated value of the distance criterion is non-zero because the widths of skin panels were intentionally adjusted to observe any solver anomalies. Wider panels have been tested and similar results were generated.

Next, 10 [deg] global z-axial twist was tested to see if the solver passes as a viable solution. The result is seen below:

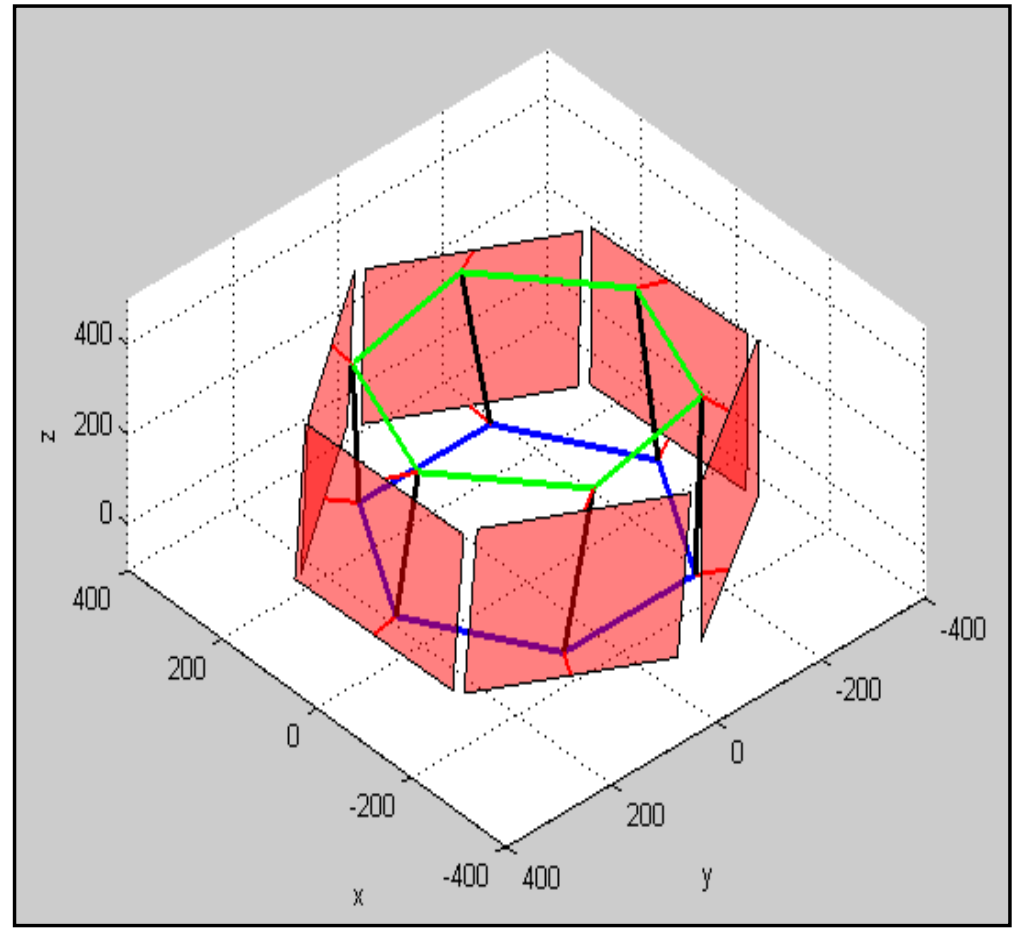

(a) 


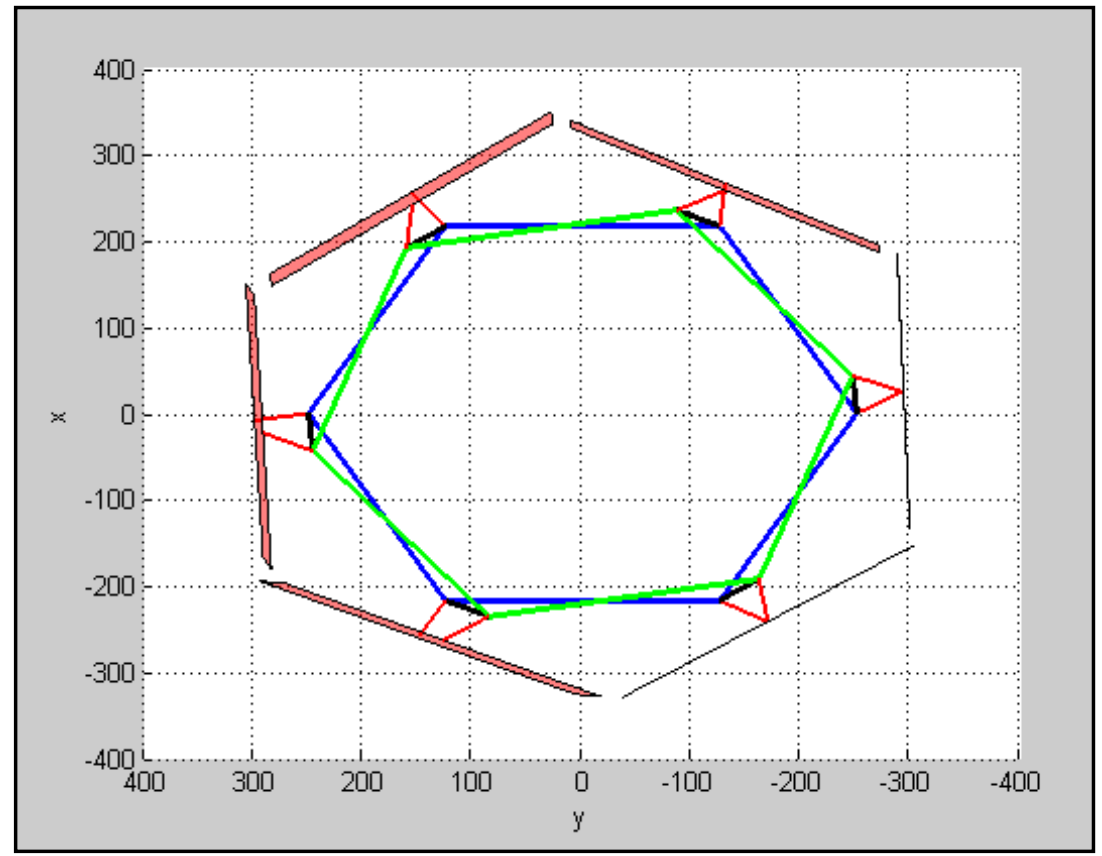

(b)

Fig. 29: Passive panel orientation with goal attainment for 10 [deg] global z-axial twists. (a) Isometric view (b) top view

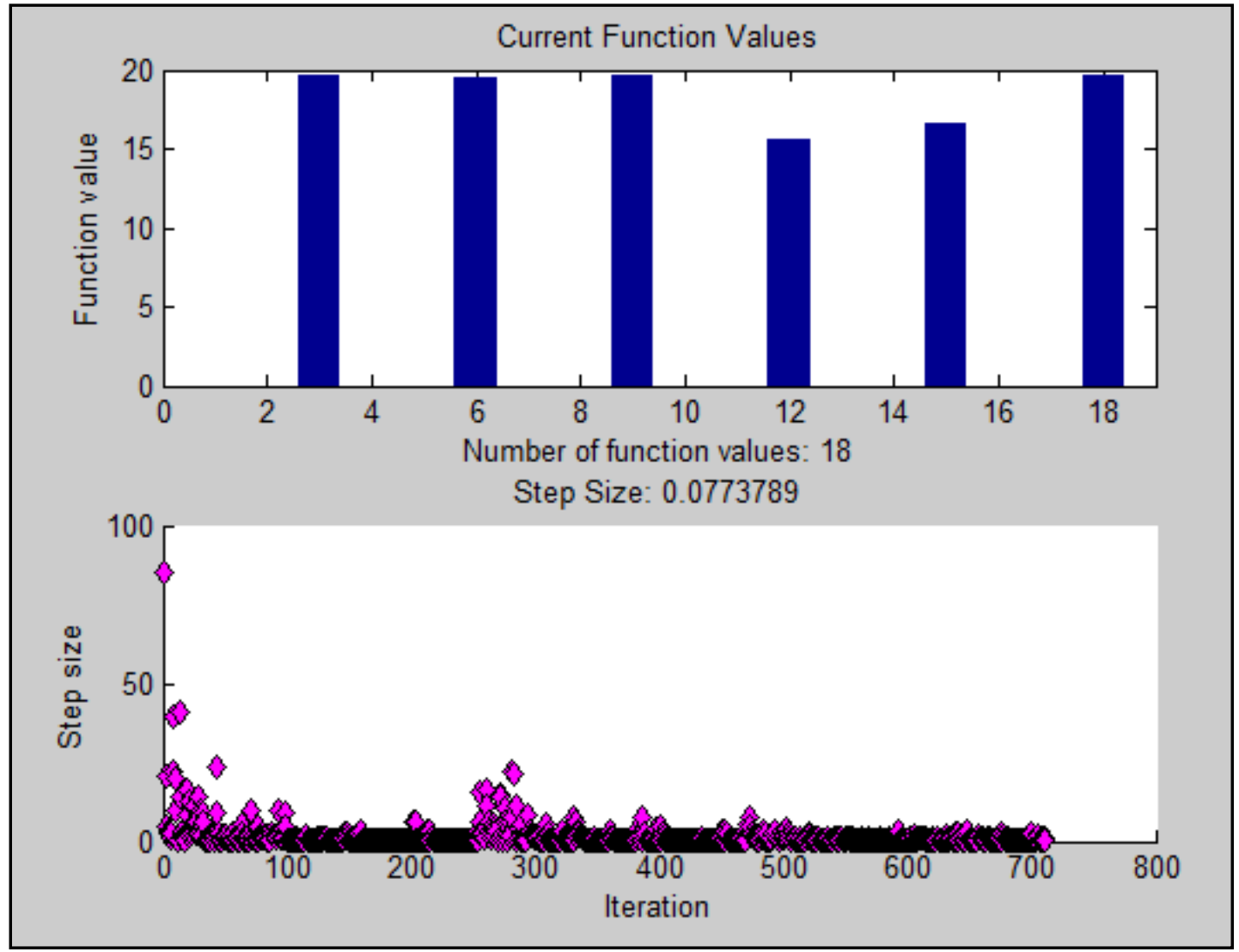

Fig. 30: Goal attainment for $10[\mathrm{deg}]$ global z-axial twist 
Table 3: Goal attainment criteria results - $10[\mathrm{deg}]$ global z-axial twist

\begin{tabular}{|c|c|c|c|c|c|c|}
\hline $\begin{array}{l}\text { Global x-axial twist: } 0[\mathrm{deg}] \\
\text { Global y-axial twist: } 0[\mathrm{deg}] \\
\text { Global z-axial twist: } 10[\mathrm{deg}]\end{array}$ & $\begin{array}{l}\text { Global x-axial translation: } 0 \\
\text { Global y-axial translation: } 0\end{array}$ \\
\hline Branch $i$ & 1 & 2 & 3 & 4 & 5 & 6 \\
\hline$m_{x, i, L R}$ & 0 & 0 & 0 & 0 & 0 & 0 \\
\hline$m_{y, i, L R}$ & 0 & 0 & 0 & 0 & 0 & 0 \\
\hline$d_{i, L R}$ & 19.59 & 19.53 & 19.62 & 15.82 & 16.54 & 19.58 \\
\hline
\end{tabular}

As seen from Fig. 29 and Fig. 30, all three criteria were almost completely achieved, with distance among panels being slightly different along the loop. As expected, when the hexapod twists, the panel distance will decrease due to contraction of structure. Although distance criterion was not fully achieved, as seen in Table 3, all panels achieved the directional vector criteria, meaning adjacent panels are parallel or near parallel. As a result, it has proven that a passive panel will allow for hexapod orientations that an active panel could not. In addition, this also displays the inferiority of the linear formulation method. With the lack of possible singularity in non-linear formulation, it is possible to achieve results for the baseline configuration, whereas linear formulation failed. The only observable issue with goal attainment is the sensitivity of the defined goal. If the goal of all three criteria were set to zero, the solver will reduce in accuracy and produce unreasonable results. However, when small values (ex. 10 ${ }^{-6}$ ) are used, the solver produces reasonable results. It was observed different orientations require different goals in order to achieve reasonable results. This was tested and demonstrated, the results are provided in Table 4 with different configurations: 
Table 4: Other configurations with goal attainment

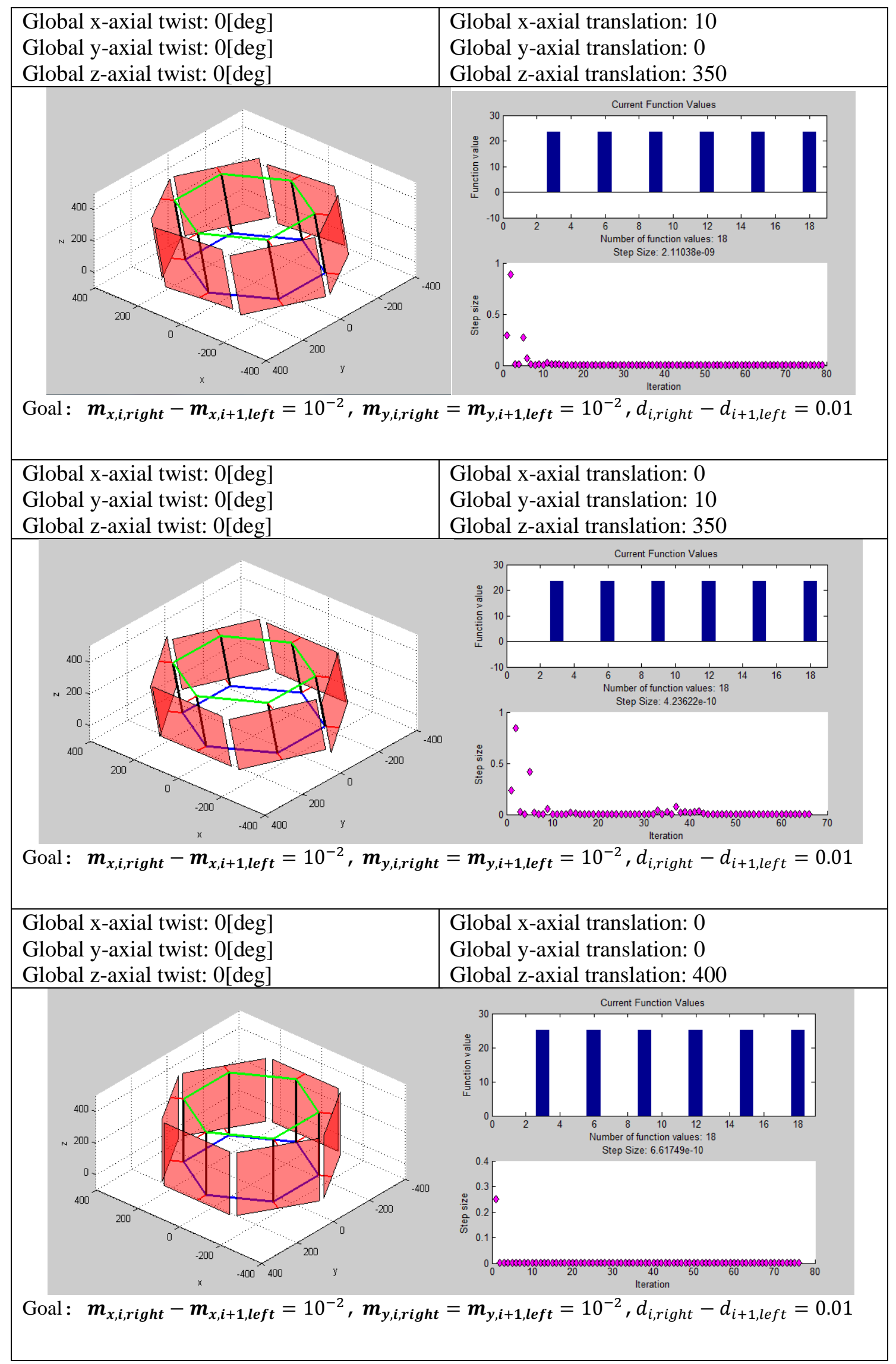




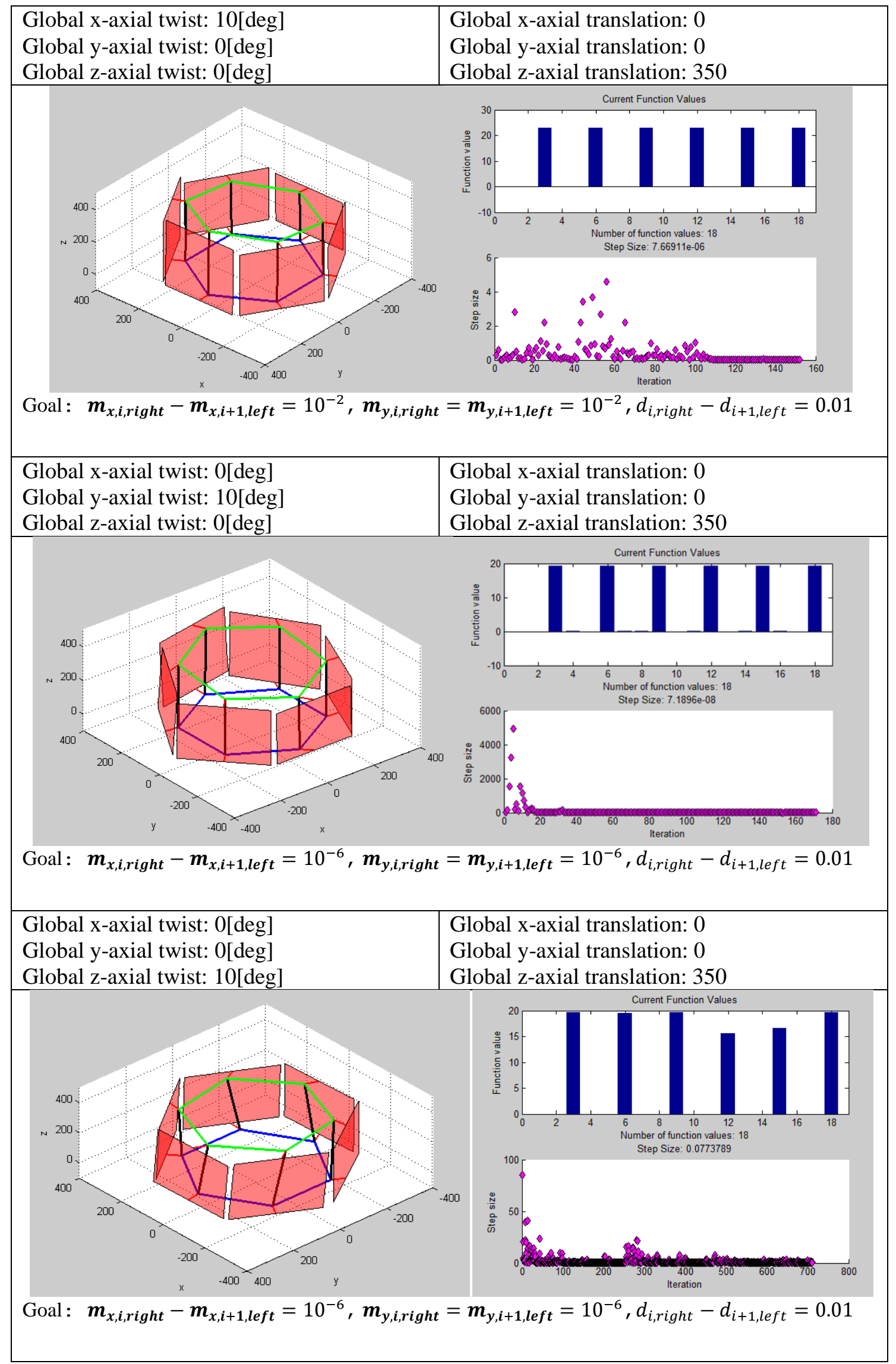




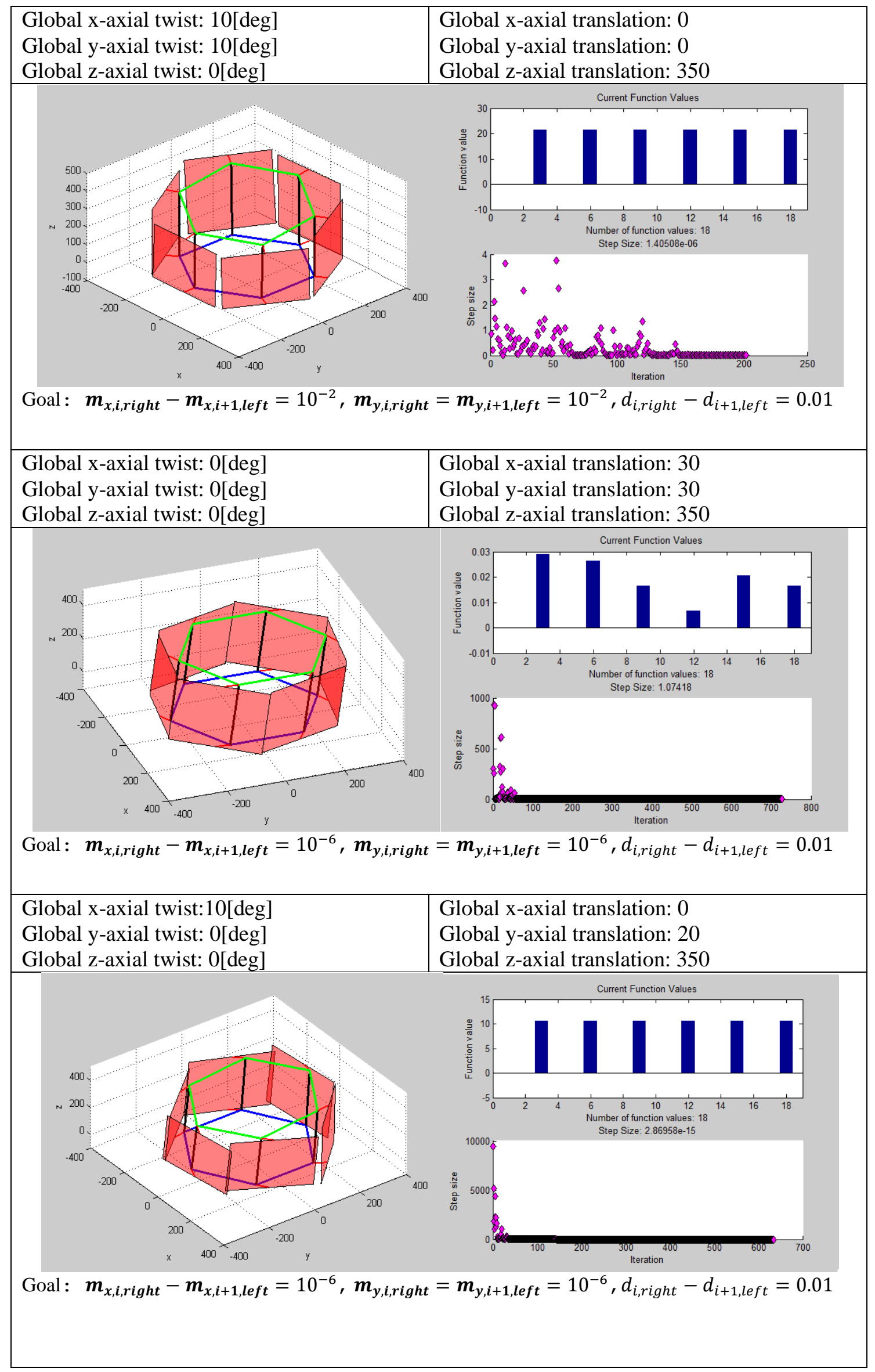




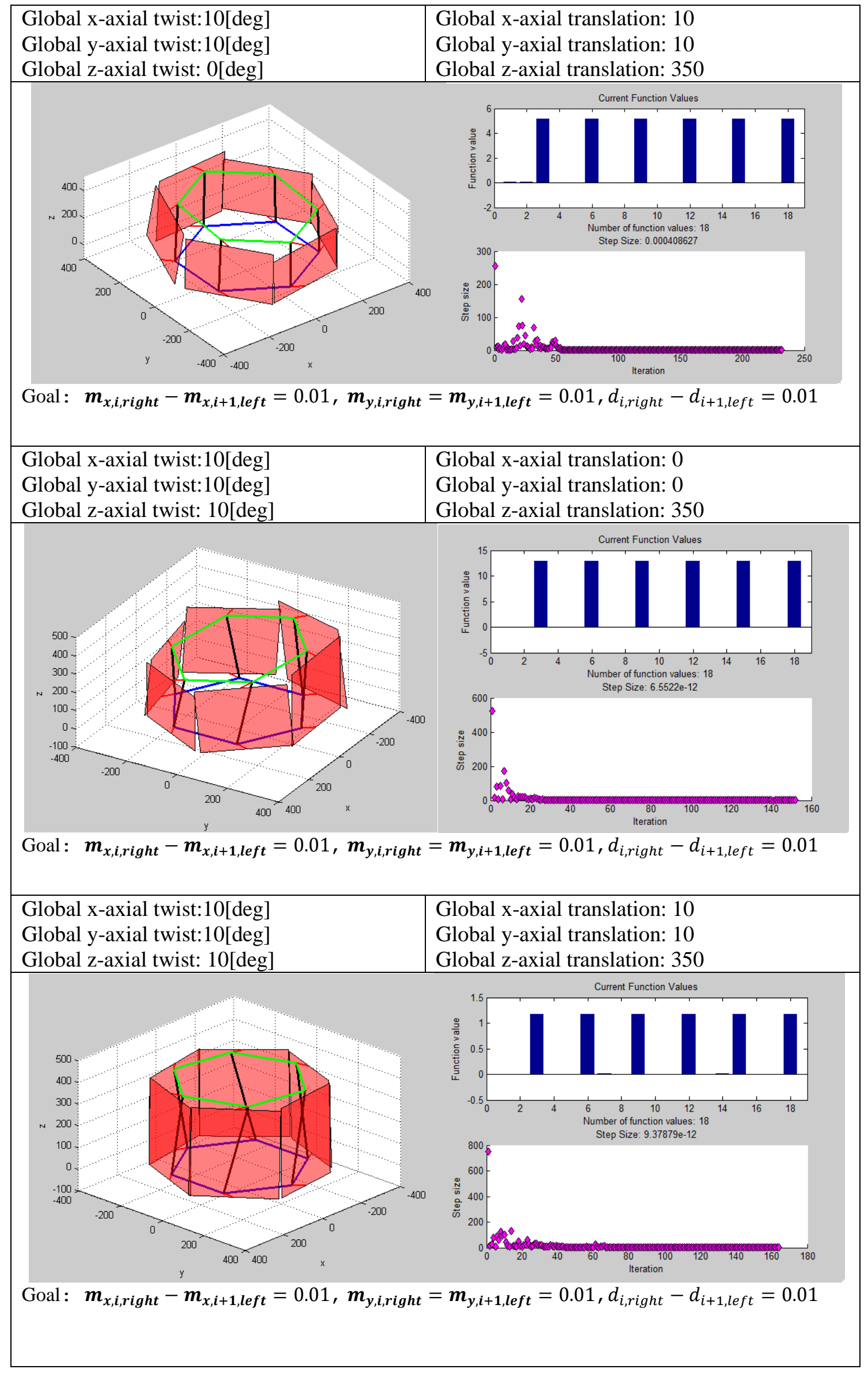




\subsubsection{MinimaX}

Similar to goal attainment, Minimax was straightforward to program. Also, the result representation and computational time are similar. However, the results itself are dissimilar. The baseline orientation was the first flag:

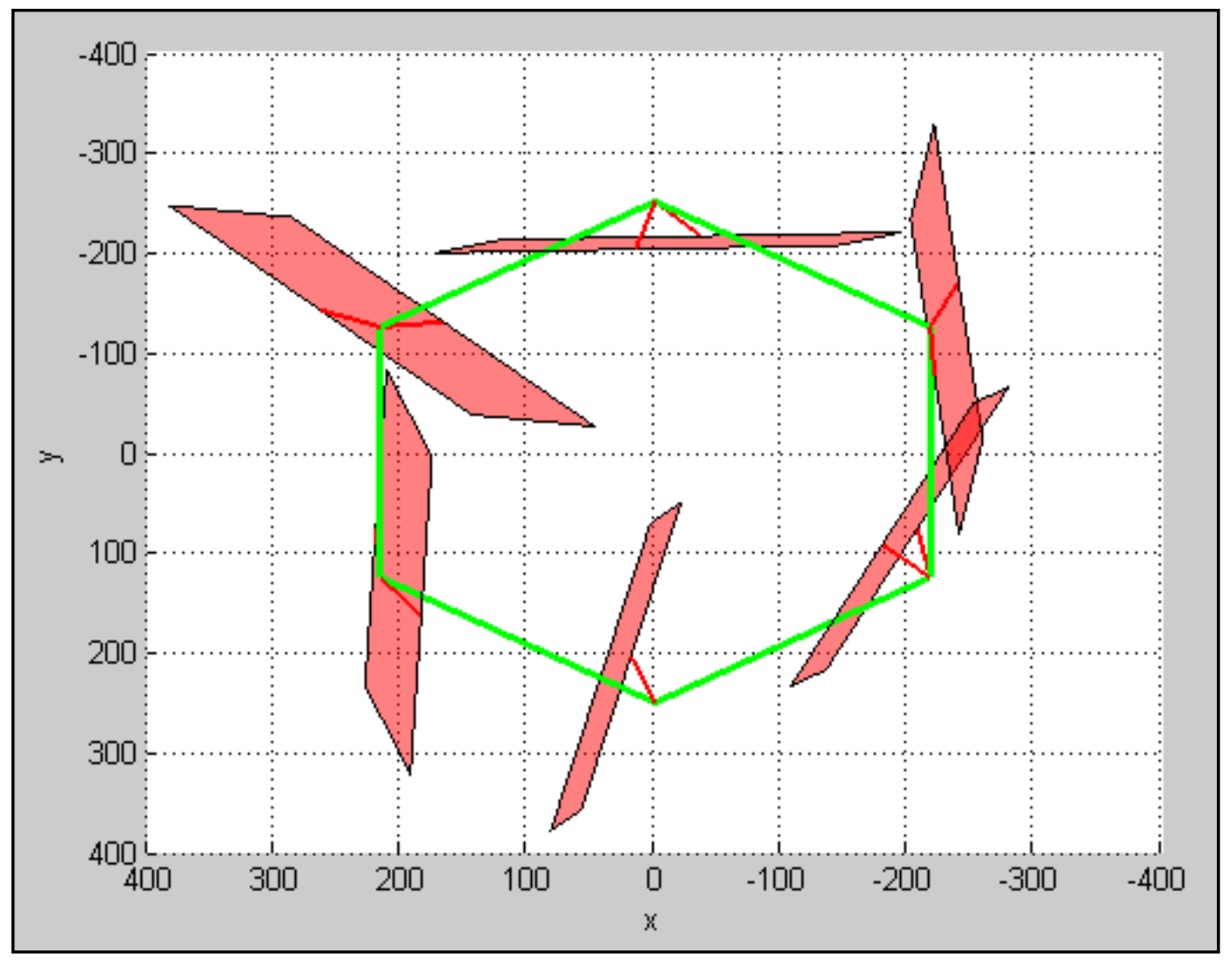

Fig. 31: Passive panel orientation with Minimax for baseline orientation

From Fig. 31, it seems as so Minimax is not a very stable function. Given initial values are all zero; the solver was expected to exit rapidly. Instead, it failed all criteria and converged to what is seen above. However, when geometric transformation was applied to the hexapod, the solver exhibited some forms of stability: 


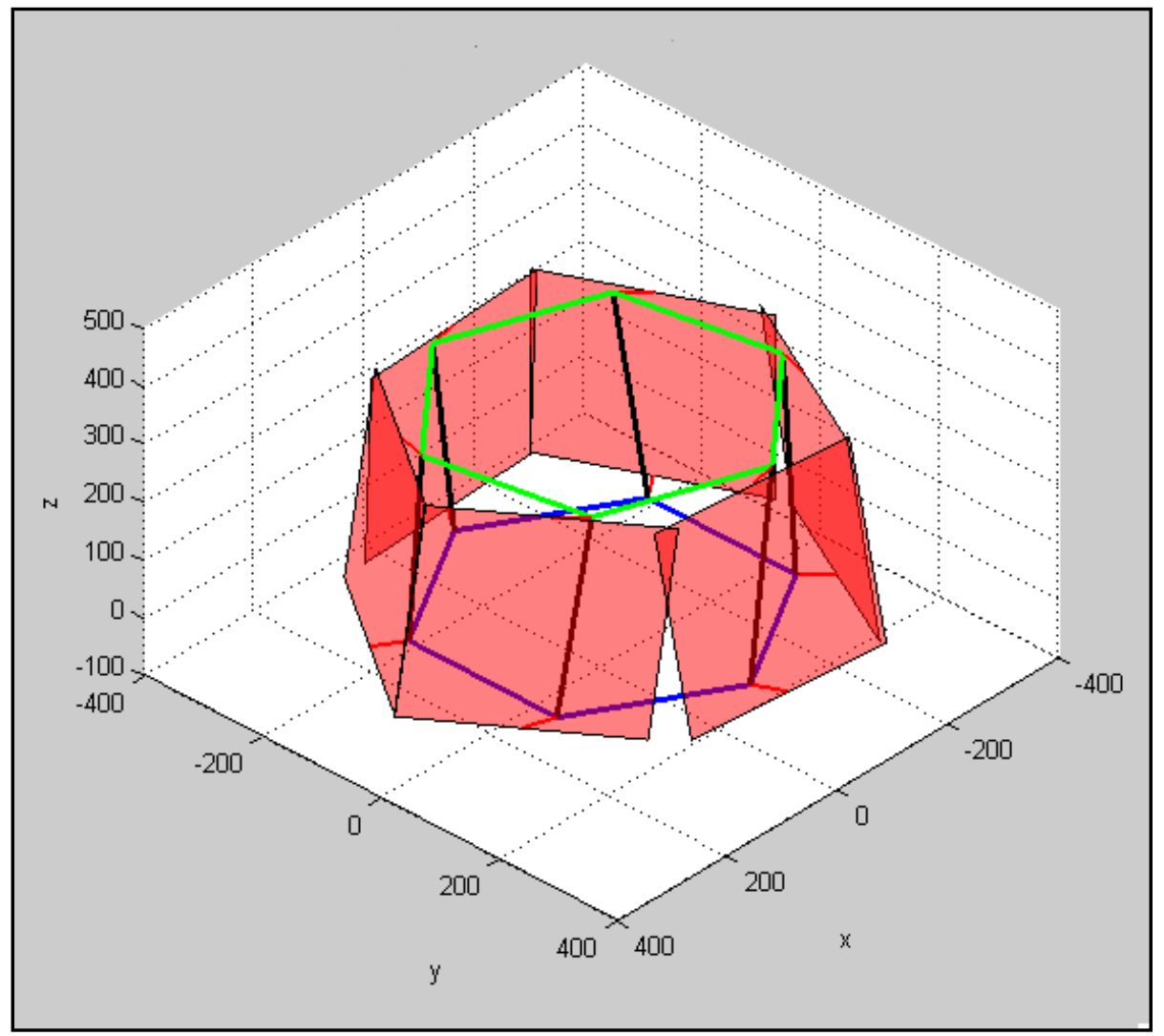

Fig. 32 Passive panel orientation with Minimax for 10 [deg] global z-axial twist

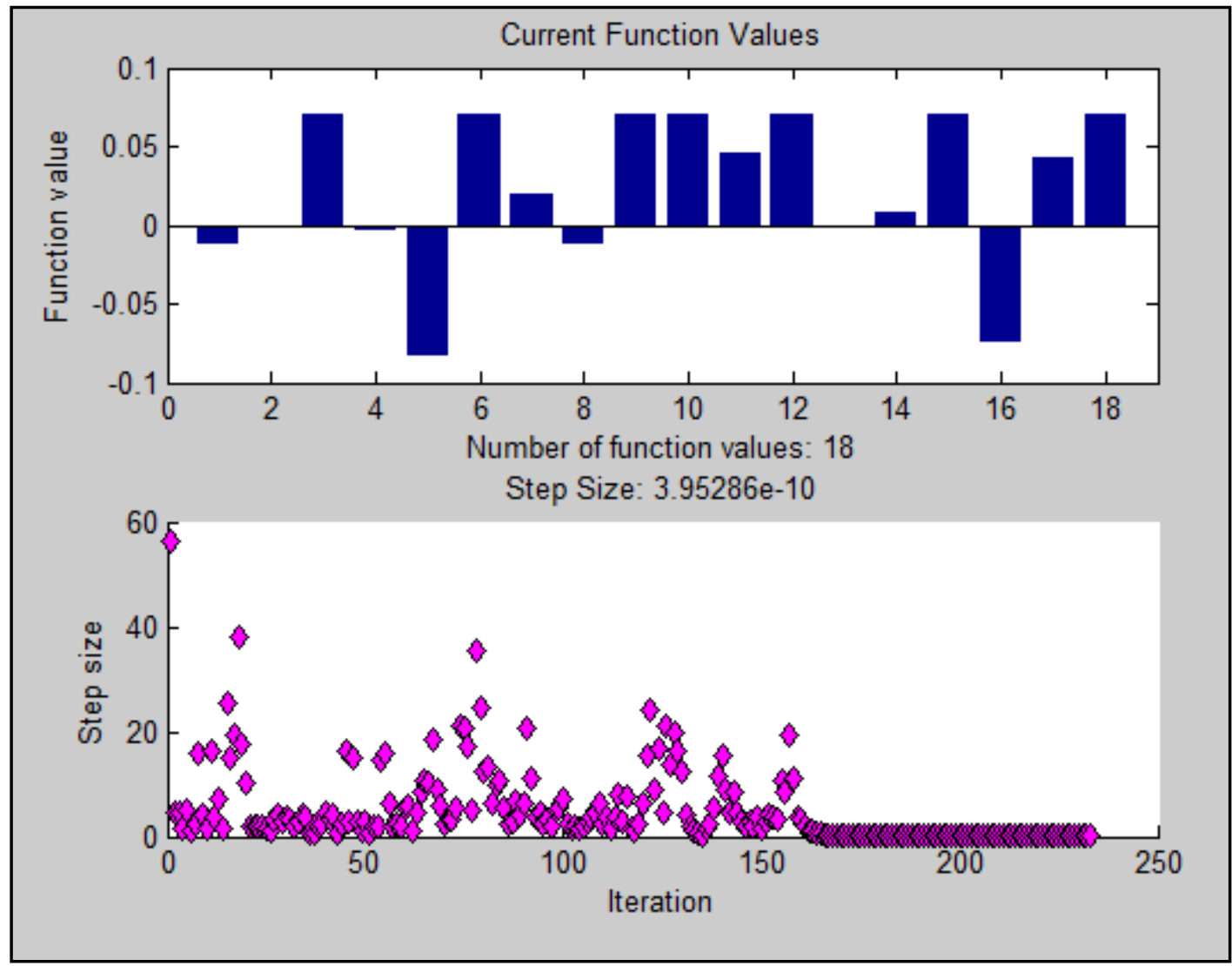

Fig. 33: Minimax for 10 [deg] global z-axial twist 
Table 5: Minimax criteria results - 10[deg] global z-axial twist

\begin{tabular}{|c|c|c|c|c|c|c|}
\hline \multicolumn{5}{|l|}{$\begin{array}{l}\text { Global x-axial twist: } 0[\mathrm{deg}] \\
\text { Global y-axial twist: } 0[\mathrm{deg}] \\
\text { Global z-axial twist: } 10[\mathrm{deg}]\end{array}$} \\
\hline Branch $i$ & 1 & 2 & 3 & 4 & 5 & 6 \\
\hline$m_{x, i, L R}$ & $-1.17 \mathrm{E}-02$ & $-3.74 \mathrm{E}-03$ & $1.92 \mathrm{E}-02$ & $7.04 \mathrm{E}-02$ & 0 & $-7.41 \mathrm{E}-02$ \\
\hline$m_{y, i, L R}$ & 0 & $-8.38 \mathrm{E}-02$ & $-1.23 \mathrm{E}-02$ & $4.58 \mathrm{E}-02$ & $7.49 \mathrm{E}-03$ & $4.27 \mathrm{E}-02$ \\
\hline$d_{i, L R}$ & $7.04 \mathrm{E}-02$ & $7.04 \mathrm{E}-02$ & $7.04 \mathrm{E}-02$ & $7.04 \mathrm{E}-02$ & $7.04 \mathrm{E}-02$ & $7.04 \mathrm{E}-02$ \\
\hline
\end{tabular}

As seen from Fig. 32 and Fig. 33, this is a good example of what happens when minimum distance criterion is reached but all else fails. In other words, it failed to achieve parallelism. In Table 5, compared to goal attainment, the distance criterion generated by Minimax is much closer to zero. This is because directional vectors are not parallel, resulting in intersections. This causes the minimum distance to appear among the intersection of adjacent panels. Therefore, with these two flags, Minimax may have potential as a solver, but debugging the issues is not within the scope of this thesis and is eliminated as a viable solution.

\subsubsection{MultiobJective Genetic Algorithm}

On paper, multiobjective GA seems to be a potential candidate to the problem of nonlinear systems of equations, since it is technically a brute force method. It runs through a potential population of solutions to obtain the final ideal solution. This results in long computational times and complex initializations and setup of program, which was indeed the case. Despite all the minor details, the main issue is the solver's ability to handle more than two objectives, which are dependant of each other. In the case of this thesis, there are eighteen objectives, all strongly dependant on each other. For gamultiobj( ) to run efficiently for multiple objectives, it is best to have independent objectives. If the objectives are strongly dependent upon each other the solver's efficiency will dramatically decrease [65]. Such can be seen with one of the solutions of the baseline orientation: 


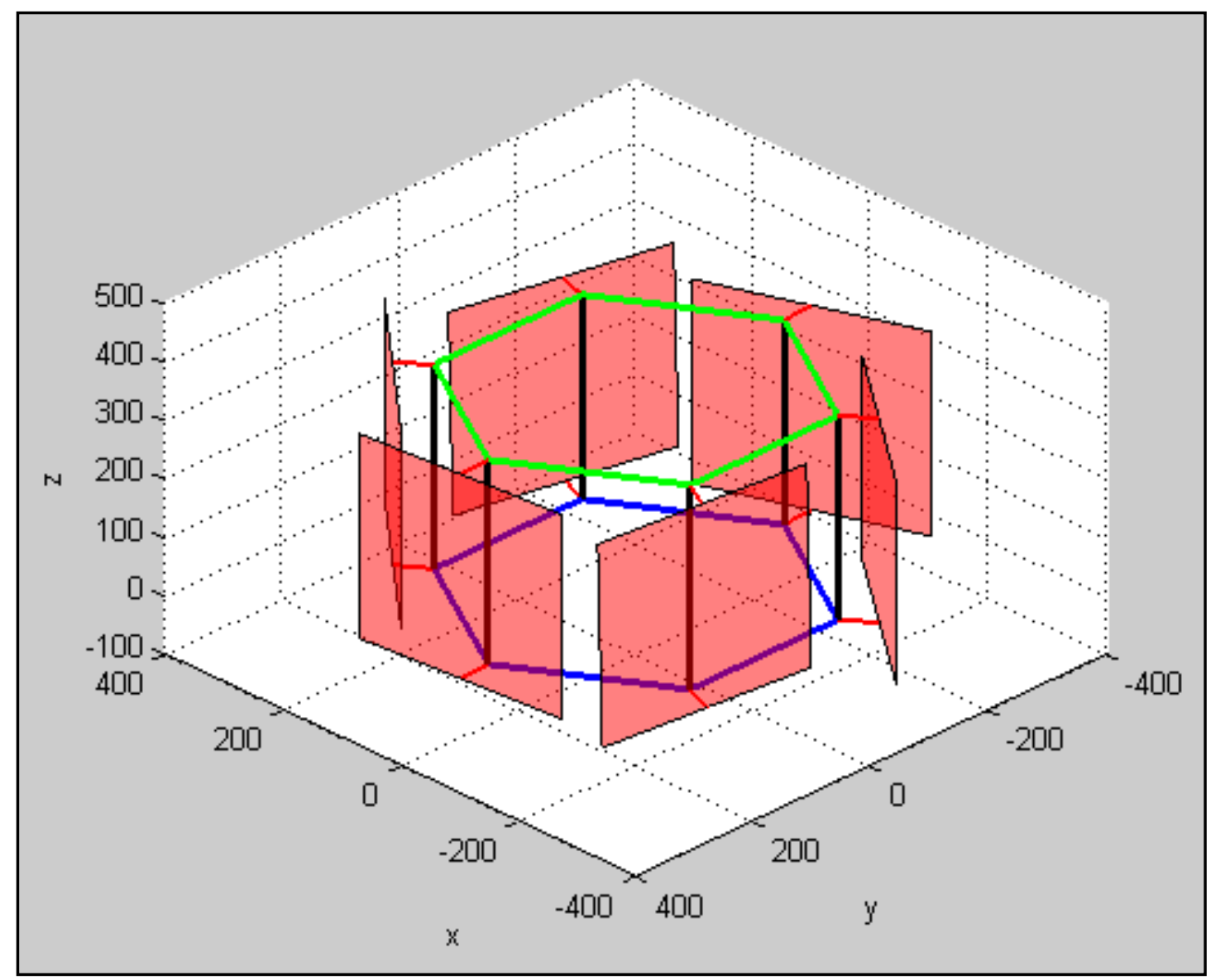

Fig. 34: Passive panel orientation with multiobjective GA for baseline orientation

In Fig. 34, the passive panel orientation is less dramatic than Minimax. Although parallelism is achieved, the distance criterion is increased instead of reduced. Another issue with multiobjective GA is that it creates multiple solutions. Fig. 34 is one of the eighteen solutions created by the solver. Out of the eighteen solutions, three solutions achieved baseline orientations. Same method was applied to the 10 [deg] global zaxial twist and the following were obtained: 


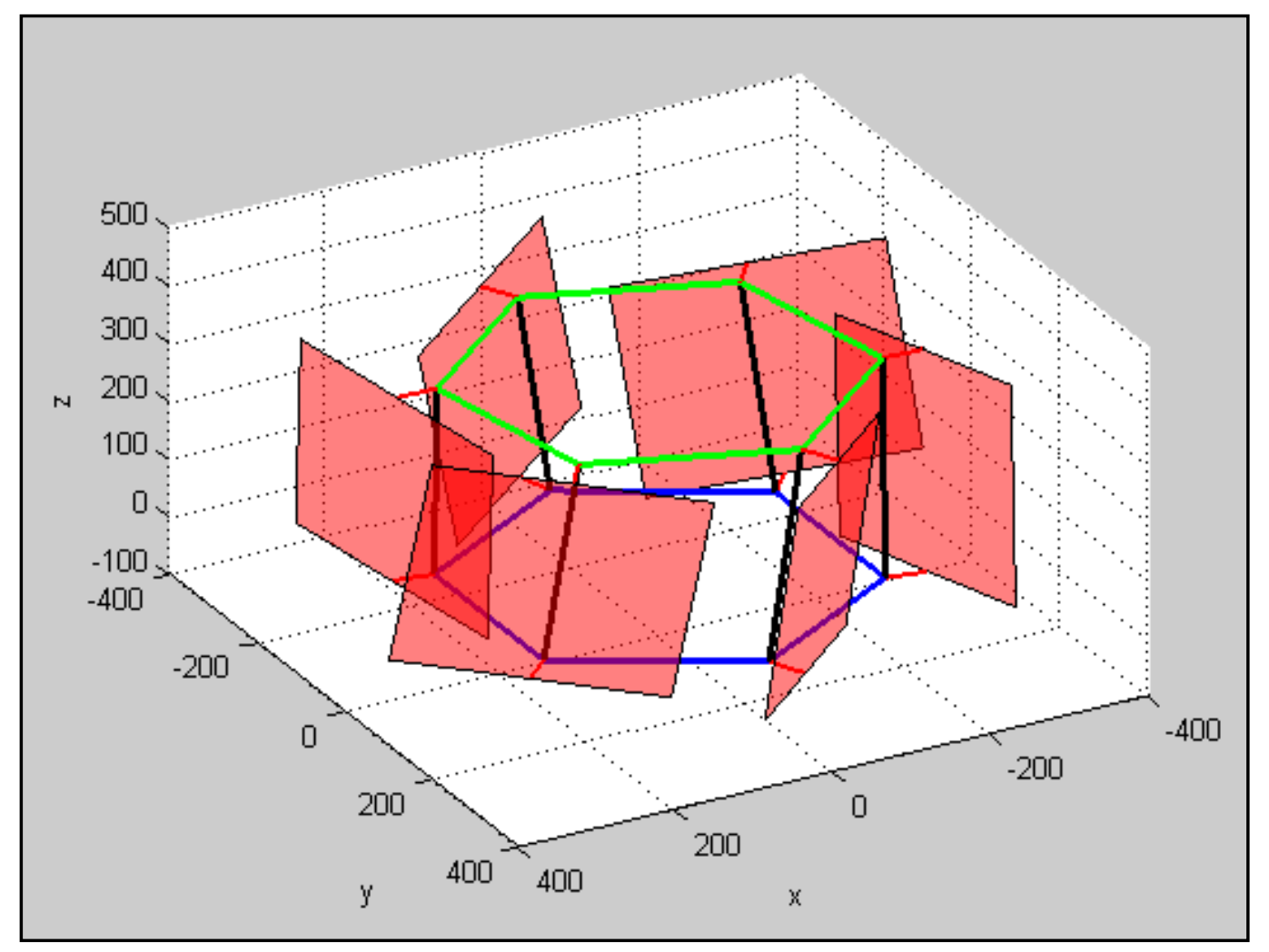

(a)

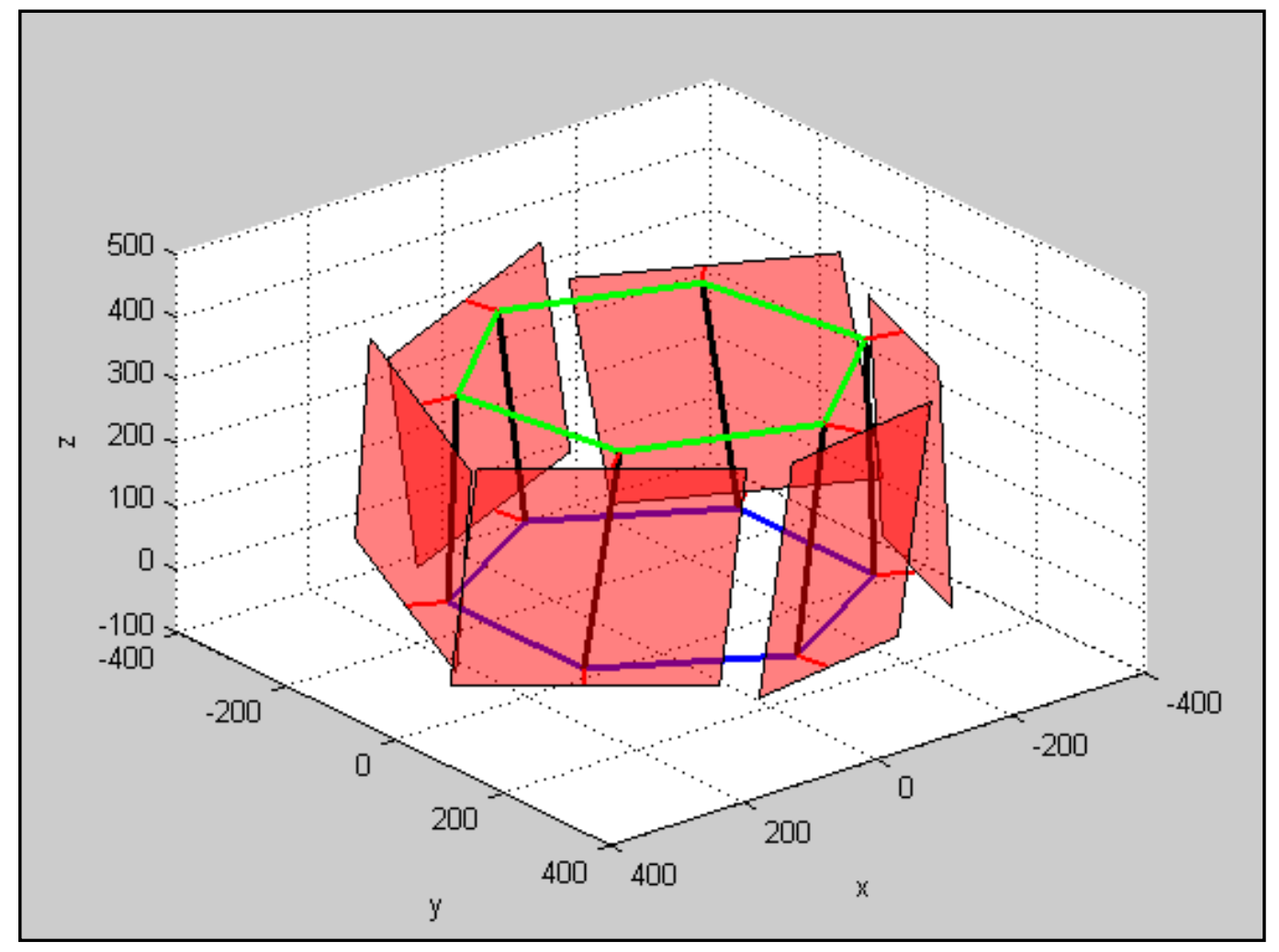

(b) 


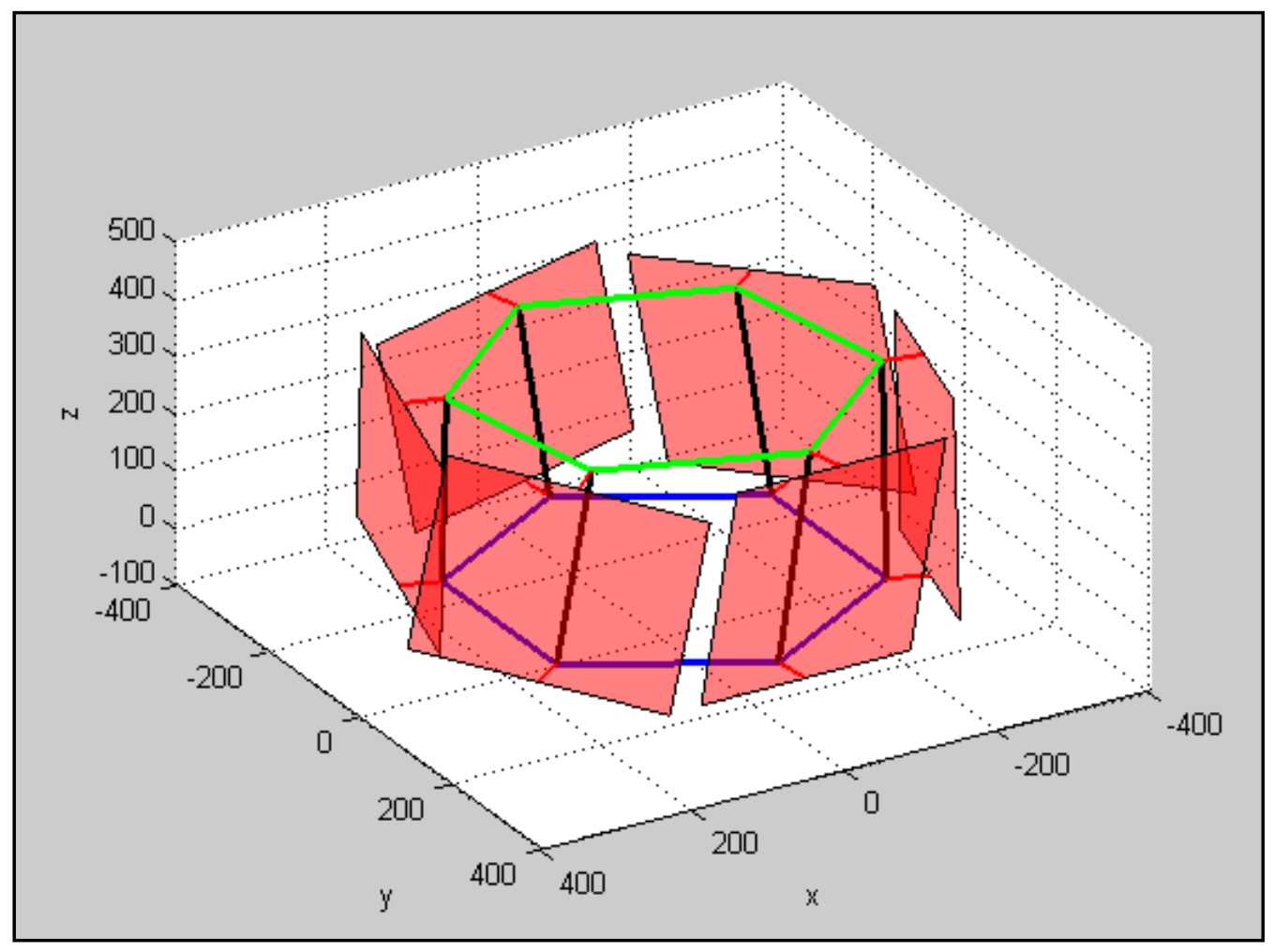

(c)

Fig. 35: Passive panel orientation with multiobjective GA for 10[deg] global zaxial twist

Table 6: Multiobjective GA criteria results - 10[deg] global z-axial twist

\begin{tabular}{|c|c|c|c|c|c|c|}
\hline \multirow{4}{*}{\multicolumn{3}{|c|}{$\begin{array}{l}\text { Global x-axial twist: } 0[\mathrm{deg}] \\
\text { Global y-axial twist: } 0[\mathrm{deg}] \\
\text { Global z-axial twist: } 10[\mathrm{deg}]\end{array}$}} & \multirow{2}{*}{\multicolumn{4}{|c|}{ Global x-axial translation: 0}} \\
\hline & & & & & & \\
\hline & & & \multicolumn{4}{|c|}{ Global y-axial translation: 0} \\
\hline & & & \multicolumn{4}{|c|}{ Global z-axial translation: 350} \\
\hline \multicolumn{7}{|l|}{ Fig. 35(a) } \\
\hline Branch $i$ & 1 & 2 & 3 & 4 & 5 & 6 \\
\hline$m_{x, i, L R}$ & 0 & $-8.84 \mathrm{E}-02$ & $-5.24 \mathrm{E}-02$ & $8.54 \mathrm{E}-02$ & $5.54 \mathrm{E}-02$ & 0 \\
\hline$m_{y, i, L R}$ & 0 & 0 & $-1.65 \mathrm{E}-02$ & $-1.07 \mathrm{E}-01$ & $-2.21 \mathrm{E}-02$ & $1.46 \mathrm{E}-01$ \\
\hline$d_{i L R}$ & 74.8 & 98.63 & 97.12 & 148.19 & 57.77 & 11.98 \\
\hline
\end{tabular}

\begin{tabular}{|c|c|c|c|c|c|c|}
\hline \multicolumn{7}{|l|}{ Fig. 35(b) } \\
\hline Branch $i$ & 1 & 2 & 3 & 4 & 5 & 6 \\
\hline$m_{x, i, L R}$ & 0 & $-8.77 \mathrm{E}-02$ & $-6.52 \mathrm{E}-02$ & $9.96 \mathrm{E}-02$ & $5.33 \mathrm{E}-02$ & 0 \\
\hline$m_{y, i, L R}$ & 0 & 0 & $-1.63 \mathrm{E}-02$ & $-9.89 \mathrm{E}-02$ & $-4.80 \mathrm{E}-03$ & $1.20 \mathrm{E}-01$ \\
\hline$d_{i, L R}$ & 15.88 & 68.06 & 35.83 & 76.23 & 26.134 & 83.94 \\
\hline
\end{tabular}

\begin{tabular}{|c|c|c|c|c|c|c|}
\hline Fig. 35(c) & \multicolumn{1}{l|}{} \\
\hline Branch $i$ & 1 & 2 & 3 & 4 & 5 & 6 \\
\hline$m_{x, i, L R}$ & 0 & $-8.22 \mathrm{E}-02$ & $-8.36 \mathrm{E}-02$ & $1.10 \mathrm{E}-01$ & $5.55 \mathrm{E}-02$ & 0 \\
\hline$m_{y, i, L R}$ & 0 & 0 & $-1.89 \mathrm{E}-02$ & $-1.01 \mathrm{E}-01$ & $1.43 \mathrm{E}-03$ & $1.19 \mathrm{E}-01$ \\
\hline$d_{i, L R}$ & 42.33 & 27.26 & 29.12 & 69.28 & 23.50 & 60.36 \\
\hline
\end{tabular}


Fig. 35 (c) was the best result out of the eighteen solutions. Three selected solutions are shown here to demonstrate the difference in results that multiobjective GA solver can produce. Numerical representation can be seen in Table 6. Even for the best result (Fig. 35 (c)), the solution fails to produce parallelism among adjacent panels and instead of decreasing panel gap distance, some instances were increased. There is a suggestion to improve solver efficiency, it involves changing the population sizes and generations, but due to generation and population size is unique to all problems. A grid study is required to select the ideal population and generation values. With the issue of solver inefficiency, multiple solutions, and noticeably longer computation time (approximately 10 to 20 [mins]), Multiobjective GA was excluded as a potential solution for this thesis. Although excluded, it may be used to search for possible solutions.

\subsection{Methodology APPLiCATion to MorPhing SkIN}

It has been mentioned previous in chapter 5; this is technically a simulation of an existing prototype that is in Ryerson University. From the simulation, it is possible to understand the interactions among top panels and bottom panels during different mechanism orientations. To increase the applicability of the simulation, it is possible to increase the number of branches and alter the shape of the mechanism. Therefore, instead of a hexapod, an airfoil shape can be used and six sliding panels can be increased to twenty panels. The most important note to make is a baseline simulation has been created to advance an all-purpose morphing skin design to more complex geometry.

From the simulation and the development of this thesis, a few things have been observed. The current prototype uses a mutually dependant sliding mechanism. With 
this mechanism, skin panels are able to hold a given shape. The down side is the limitation to twist, as presented in chapter 5. The prototype was in a $10[\mathrm{deg}]$ twist, but it was only possible because independent panels were passively twisted. Twisting was possible because the material used to manufacture the prototype is elastic. If the prototype was manufactured with solid steel, a $10[\mathrm{deg}]$ twist is not possible. Therefore, two solutions are available. One, use materials with high elasticity while providing sufficient amount of stiffness to sustain bending and external loadings. Two, design a compliant joint sliding panel based on the simulation provided in this thesis to accept twisting motions, resulting in a complex hybrid design. Another observation made was that solid flat panels are not a realistic design. Most mechanism require a shelllike structures of a multitude of different geometries, if a flat panel were to achieve said geometry, it will require extremely fine discretization of the geometry, which will result in large amounts of slim panels. This alone is not structurally viable and is not advised. So it is recommended to incorporate curved or differential surfaces into the simulation. The final observation made was there are many unknowns in this method, this is due to the study of such interaction is completely unique and new. Some notable ambiguities in this research include the ideal link length for effective passive panel configuration, the ideal optimization algorithm constraints to obtain reasonable results, the ideal telescopic mechanism to compensate for sliced active panels, and among many others.

With the baseline simulation complete, it is possible to address all of the above unknowns in future works and begin the development of an improved morphing skin design. 


\section{CHAPTER 7}

CONCLUSION AND FUTURE WORK

\subsection{CONCLUSION}

In this thesis, a simulation for a sliding panel morphing skin design was made and panel interactions were analyzed. Two methods were suggested in this thesis to answer the interactions among adjacent panels. First method is an active panel method, where the panels are attached directly to the branches of the mechanism, where the branches actively determine the orientation of the panels. A simulation is developed as a prognostic device to tailor panel geometry to fit certain orientations of the mechanism. A panel optimization algorithm is developed for this purpose. The algorithm will tailor individual skin panels based on the mechanisms designed workspace. The second method is a passive panel method, where the panels are extended by jointed links. Instead of being directly driven by the actuators of the mechanism, the skin panels will reorientate based on the orientation of the internal mechanism and geometrical constraints surrounding skin panels. The final orientation will be free of collisions or intersections among adjacent panels. An optimization method for panel orientation is developed for this purpose. The optimization results may be summarized with the following table:

Table 7: Summary of optimization methods

\begin{tabular}{|c|c|c|c|}
\cline { 2 - 4 } \multicolumn{1}{c|}{} & Baseline configuration & $\mathbf{1 0}[\mathbf{d e g}$ z-axial twist & Computation time \\
\hline attainment & $\begin{array}{c}\text { Converged with } \\
\text { expected results }\end{array}$ & $\begin{array}{c}\text { Converged with } \\
\text { expected results }\end{array}$ & $\sim 30-60[\mathrm{secs}]$ \\
\hline Minimax & Fail & $\begin{array}{c}\text { Converged with poor } \\
\text { results }\end{array}$ & $\sim 30-60[\mathrm{secs}]$ \\
\hline $\begin{array}{c}\text { Multiobjective } \\
\text { GA }\end{array}$ & $\begin{array}{c}\text { Converged with } \\
\text { multiple results, only } \\
16 \% \text { of the results are } \\
\text { acceptable }\end{array}$ & $\begin{array}{c}\text { Converged with } \\
\text { multiple results, only } \\
16 \% \text { of the results are } \\
\text { acceptable }\end{array}$ & $\sim 10-20$ [mins] \\
\hline
\end{tabular}


From Table 7, goal attainment appears to be ideal method to solving the multiobjective problem of this thesis. Since it provides expected results and has a relatively low computational time compared to the other two methods. Minimax will not be further discussed or looked upon in the future, due to the solver instability. However, Multiobjective GA will be reconsidered and investigated as a potential solution method. This is because the solver is capable of generating multiple solutions, some of which deemed viable as a solution to the given non-linear system. Even if it is not viable as a solver, it may be used to search for possible alternate solutions. In addition, observations were made during the investigation of optimization algorithms. Certain mechanism orientations for passive panels are faster to solve, with less differentiation among all six the distance criteria. Orientations include $\mathrm{x}, \mathrm{y}, \mathrm{z}$-axial translation, symmetrical $\mathrm{x}$, and $\mathrm{y}$-axial twisting. This is because parallelism is naturally maintained by the physical design of the system, thus the aforementioned orientations will not cause parallelism to be broken in any form. However, when the axis of symmetry for $\mathrm{x}$ and $\mathrm{y}$-axial twisting are not between two opposing branches, parallelism is harder to achieve since this would introduce the possible cascading of adjacent panels. This is similar to global z-axial twist and therefore asymmetrical $\mathrm{x}, \mathrm{y}$ axial twist, and global z-axial twist is the most difficult to solve. Overall, the two developed methods will serve as a baseline methodology for improving future designs of the sliding panel morphing skin.

\subsection{CONTRIBUTIONS}

The following contributions are made in this thesis: 
1. Kinematic modeling of panel enclosed mechanism has been developed. A program that fully simulates a six DOF mechanism enclosed within morphing rigid panel configuration.

2. Formulated numerical relations among closed loop panel-to-panel interactions. From numerical formulations, two methods for resolving close proximity panel collisions were proposed, simulated, and tested. The two methods include active panel and passive panel.

3. Linear and non-linear methods were investigated for solving a large mutually dependant passive panel system.

4. Created a baseline simulation program that can be modified to emulate an existing morphing skin design at Ryerson University. The formulas and simulations presented in this thesis will help improve the overall design.

\subsection{FUTURE WORK}

As mentioned in chapter 7, there are a few things that can be investigated in future works. Most importantly, the implementation of the simulation on differential surfaces is top priority. Other improvements include improving the optimization techniques, such as finding the ideal initialization values for consistent solver robustness. Next is investigating panels with elasticity to diverge from using a fully rigid panel, to resolve the bending issue with the mutually dependent sliding panel configuration. Finally, to increase panel numbers and modify mechanism to fit any form of morphing mechanism. 


\section{REFERENCES}

1. Bi, Z.M. and Jin, Y., "Kinematic Modeling of Exechon Parallel Kinematic Machine". Robotics and Computer-Integrated Manufacturing, 2011. 27: p. 186-193.

2. Makoto, K., "Twin-Head Six-Axis Force Sensors". IEEE Transactions on Robotics and Automation, 1996. 12(1): p. 146-154.

3. Ferre, M., Galiana, I., Wirz, R., and Tuttle, N., "Haptic Device for Capturing and Simulating Hand Manipulation Rehabilitation". IEEE/ASME Transactions on Mechatronics, 2011. 16(5): p. 808-815.

4. Cheng, G., Xu, P., Yang, D., and Liu, H., "Stiffness Analysis of A 3CPS Parallel Manipulator For Mirror Active Adjusting Platform in Segmented Telescope". Robotics and Computer-Integrated Manufacturing, 2013. 29: p. 302-311.

5. Finistauri, A.D. and Xi, F., "Type Synthesis and Kinematics of a Modular Variable Geometry Truss Mechanism for Aircraft Wing Morphing". ASME/IFToMM International Conference on Reconfigurable Mechanisms and Robots: p. 478-485.

6. Moosavian, A., Xi, F., and Hashemi, S.M., "Design and Motion Control of Fully Variable Morphing Wings". Journal of Aircraft, 2013. 50(4): p. 11891201.

7. Olympio, K.R. and Gandhi, F., "Flexible Skins for Morphing Aircraft Using Cellular Honeycomb Cores". Journal of Intelligent Material Systems and Structures, 2010. 21: p. 1719-1735.

8. Olympio, K.R., Gandhi, F., Asheghian, L., and Kudva, J., "Design of A Flexible Skin for A Shear Morphing Wing". Journal of Intelligent Material Systems and Structures, 2010. 21: p. 1755-1770.

9. Bubert, E.A., Woods, B.K.S., Lee, K., Kothera, C.S., and Wereley, N.M., "Design and Fabrication of a Passive 1D Morphing Aircraft Skin". Journal of Intelligent Material Systems and Structures, 2010. 21: p. 1699-1717. 
10. Barbarino, S., Gandhi, F., and Webster, S.D., "Design of Extendable Chord Sections for Morphing Helicopter Rotor Blades". Journal of Intelligent Material Systems and Structures, 2011. 22: p. 891-905.

11. Galantai, V.P., Sofla, A.Y.N., Meguid, S.A., Tan, K.T., and Yeo, W.K., "BioInspired Wing Morphing for Unmanned Aerial Vehicles Using Intelligent Materials". Springer Science+Business Media, 2011: p. 71-79.

12. Wada, B.K., Fanson, J.L., and Crawley, E.F., "Adaptive Structures". Technomic Publishing Co. Inc., 1990. 1: p. 157-174.

13. Smith, A.M.O., "High-Lift Aerodynamics". Journal of Aircraft, 1975. 12(6): p. 501-530.

14. Balaji, R., Bramkamp, F., Hesse, M., and Ballmann, J., "Effect of Flap and Slat Riggings on 2-D High-Lift Aerodynamics". Journal of Aircraft, 2006. 43(5): p. 1259-1271.

15. Shabibi, A.A., Hammadi, M.A., and Mathew, J., "Study of an Airfoil with a Flap and Spoiler". Journal of Aircraft, 1994. 31(6): p. 1324-1327.

16. Raymer, D.P., Aircraft Design: A Conceptual Approach Fourth Edition. 2006, Virginia: American Institute of Aeronautics and Astronautics, Inc.

17. Pecora, R., Amoroso, F., and Lecce, L., "Effectiveness of Wing Twist Morphing in Roll Control". Journal of Aircraft, 2012. 49(6): p. 1666-1674.

18. Raither, W., Heymanns, M., Bergamini, A., and Ermanni, P., "Morphing Wing Structure with Controllable Twist Based on Adaptive Bending-Twist Coupling". IOP Publishing Smart Materials and Structures, 2013. 22.

19. Grant, D.T., Abdulrahim, M., and Lind, R., "Flight Dynamics of A Morphing Aircraft Utilizing Independent Multiple-Joint Wing Sweep". International Journal of Micro Air Vehicles, 2010. 2(2): p. 91-106.

20. Sabri, F. and Meguid, S.A., "Flutter Boundary Prediction of an Adaptive Morphing Wing for Unmanned Aerial Vehicle". Springer Science+Business Media., 2011: p. 307-312.

21. Bae, J.-S., Seigler, M.T., Inman, D.J., and Lee, I., "Aerodynamic and Aeroelastic Considerations of A Variable-Span Morphing Wing". 
AIAA/ASME/ASCE/AHS/ASC Structures, Structural Dynamics \& Materials Conference, 2004(45).

22. Vale, J., Leite, A., Lau, F., and Suleman, A., "Aero-Structural Optimization and Performance Evaluation of a Morphing Wing with Variable Span and Camber". Journal of Intelligent Material Systems and Structures, 2011. 22: p. 1057-1073.

23. Abdulrahim, M. and Lind., R., "Control and Simulation of A Multi-Role Morphing Micro Air Vehicle". AIAA Guidance, Navigation, and Control Conference and Exhibit, 2005: p. 12-18.

24. Abdulrahim, M. and Lind., R., "Flight Testing and Response Characteristics of A Variable Gull-Wing Morphing Aircraft". AIAA Guidance, Navigation, and Control Conference and Exhibit, 2004: p. 16-19.

25. Min, Z., Kien, V.K., and Richard, L.J.Y., "Aircraft Morphing Wing Concepts with Radical Geometry Change". The IES Journal Part A: Civil \& Structural Engineering, 2010. 3(3): p. 188-195.

26. Gomez, J.C. and Garcia, E., "Morphing Unmanned Aerial Vehicles". IOP Publishing Smart Materials and Structures, 2011. 20.

27. Manzo, J. and Garcia, E., "Methodology for Design of an Active Rigidity Joint". Journal of Intelligent Material Systems and Structures, 2009. 20: p. 311-327.

28. Brailovski, V., Terriault, P., Georges, T., and Coutu, D., "SMA Actuators for Morphing Wings". Physics Procedia, 2010. 10: p. 197-203.

29. Wang, Q., Xu, Z., and Zhu, Q., "Structural Design of Morphing Trailing Edge Actuated by Sma". Front. Mech. Eng., 2013. 8: p. 268-275.

30. Xi, F., Li, Y., and Wang, H., "A Module-Based Method for Design and Analysis of Reconfigurable Parallel Robots". IEEE International Conference on Mechatronics and Automation, 2010: p. 627-632.

31. Finistauri, D.A. and Xi., F., "Reconfiguration Analysis of Fully Reconfigurable Parallel Robot". Journal of Mechanisms and Robotics, 2013. 5.

32. Thill, C., Etches, J., Bond, I., Potter, K., and Weaver, P., "Morphing Skins". The Aeronautical Journal, 2008: p. 1-23. 
33. Qiu, J., Wang, C., Huang, C., Ji, H., and Xu, Z., "Smart Skin and Actuators for Morphing Structures". Procedia IUTAM, 2014. 10: p. 427-441.

34. Vocke III, R.D., Kothera, C.S., WOods, B.K.S., and Wereley, N.M., "Development and Testing of a Span-Extending Morphing Wing". Journal of Intelligent Material Systems and Structures, 2011. 22: p. 879-890.

35. Liu, W., Zhu, H., Zhou, S., Bai, Y., Wang, Y., and Zhao, C., "In-Plane Corrugated Cosine Honeycomb for 1D Morphing Skin and its Application on Variable Camber Wing". Chinese Journal of Aeronautics, 2012. 26(4): p. 935942.

36. Joo, J.J., Reich, G.W., and Westfall, J.T., "Flexible Skin Development for Morphing Aircraft Applications via Topology Optimization". Journal of Intelligent Material Systems and Structures, 2009. 20: p. 1969-1985.

37. Thill, C., Etches, J.A., Bond, I.P., Potter, K.D., and Weaver, P.M., "Composite Corrugated Structures for Morphing Wing Skin Applications". Smart Materials and Structures, 2010. 19.

38. Tachi, T., Simulation of Rigid Origami. Tokyo.

39. Gattas, J.M. and You, Z., "Geometric Assembly of Rigid-Foldable Morphing Sandwich Structures". Elsevier: Engineering Structures, 2015. 94: p. 149-159.

40. Saito, K., Agnese, F., and Scarpa, F., "A Cellular Kirigami Morphing Wingbox Concept". Journal of Intelligent Material Systems and Structures, 2011. 22: p. 935-944.

41. Zhang, K., Qiu, C., and Jian, D.S., "Helical Kirigami-Enabled CentimeterScale Worm Robot with Shape-Memory-Alloy Linear Actuators". Journal of Mechanisms and Robotics, 2015. 7.

42. Barbarino, S., Bilgen, O., Ajaj, R.M., Friswell, M.I., and Inman, D.J., "A Review of Morphing Aircraft". Journal of Intelligent Material Systems and Structures, 2011. 22: p. 823-877.

43. Murray, G., Gandhi, F., and Bakis, C., "Flexible Matrix Composite Skins for One-dimensional Wing Morphing". Journal of Intelligent Material Systems and Structures, 2010. 21: p. 1771-1781. 
44. Murugan, S. and Friswell, M.I., "Morphing Wing Flexible Skins with Curvilinear Fiber Composites". Elsevier: Composite Structures, 2013. 99: p. 69-75.

45. Murugan, S., Flores, E.I.S., Ashikari, S., and Friswell, M.I., "Optimal Design of Variable Fiber Spacing Composites for Morphing Aircraft Skins". Elsevier: Composite Strictures, 2012. 94: p. 1626-1633.

46. Thuwis, G.A.A., Abdalla, M.M., and Gurdal, Z., "Optimization of A VariableStiffness Skin for Morphing High-Lift Devices". Smart Materials and Structures, 2010. 19.

47. Rediniotis, O.K., Wilson, L.N., Lagoudas, D.C., and Khan, M.M., "Development of a Shape-Memory-Alloy Actuated Biomimetic Hydrofoil". Journal of Intelligent Material Systems and Structures, 2002. 13: p. 35-49.

48. Gandhi, F. and Anusonti-Inthra, P., "Skin Design Studies for Variable Camber Morphing Airfoils". Smart Materials and Structures, 2008. 17.

49. Courchesne, S., Popov, A.V., and Botez, R.M., "New Aeroelastic Studies for A Morphing Wing". INCAS Bulletin, 2012. 4(2): p. 19-28.

50. Wu, J., Li, J., and Yan, S., "Design of Deployable Bistable Structures for Morphing Skin and its Structural Optimization". Engineering Optimization, 2013. 46(5): p. 745-762.

51. Yin, W., Sun, Q., Zhang, B., Liu, J., and Leng, J., "Seamless Morphing Wing with SMP Skin". Advanced Materials Research, 2008. 47-50: p. 97-100.

52. Yin, W., Liu, J., and Leng, J., "Deformation Analysis of Shape Memory Polymer for Morphing Wing Skin Under Airflow". Higher Education Press and Springer-Verlag, 2009. 4(4): p. 447-449.

53. Chen, Y., Yin, W., Liu, Y., and Leng, J., "Structural Design and Analysis of Morphing Skin Embedded With Pneumatic Muscle Fibers". Smart Materials and Structures, 2011. 20.

54. Teschner, M., Kimmerle, S., Heidelberger, B., Zachmann, G., Raghupathi, L., Fuhrmann, A., Cani, M.P., Faure, F., Magnenat-Thalmann, N., Strasser, W., and Volino, P., "Collision Detection for Deformable Objects". Computer Graphics Forum, 2005. 24(1): p. 61-81. 
55. Zhao, W. and Sun, J., "Collision Detection Research for Deformable Objects". IEEE, 2012: p. 557-561.

56. Lysenko, M., "Fourier Collision Detection". The International Journal of Robotics Research, 2013. 32(4): p. 483-503.

57. Barnhill, R.E., Farin, G., Jorban, M., and Piper, B.R., "Surface/Surface Intersection". Computer Aided Geometric Design, 1987. 4: p. 3-16.

58. Patrikalakis, N.M., "Surface-to-Surface Intersections". IEEE Computer Graphics \& Applications, 1993: p. 89-95.

59. Elber, G., Grandine, T., and Kim, M.-S., "Surface Self-Intersection Computation via Algebraic Decomposition". Elsevier Computer-Aided Design, 2009. 41: p. 1060-1066.

60. Fioravanti, M., Gonzalez-Vega, L., and Necula, I., "Computing The Intersection of Two Ruled Surfaces By Using A New Algebraic Approach". Elsevier Journal of Symbolic Computation, 2006. 41: p. 1187-1205.

61. Wu, S.-T. and Andrade, L.N., "Marching Along A Regular Surface/Surface Intersection with Circular Steps". Elsevier Computer Aided Geometric Design, 1999. 16: p. 249-268.

62. Ye, X. and Maekawa, T., "Differential Geometry of Intersection Curves of Two Surfaces". Elsevier Computer Aided Geometric Design, 1999. 16: p. 767788.

63. Burger, H. and Schaback, R., "A Parallel Multistage Method for Surface/Surface Intersection". A Computer Aided Geometric Design, 1993. 10: p. 277-291.

64. Xi, F., Walsh, P., and Behdinan, K., Development of an Aircraft Morphing Wing Using Variable Geometry Truss Mechanisms, 2013, Ryerson University: Technical report to Bombardier

65. The MathWorks, I. MathWorks 2015 June; Available from: http://www.mathworks.com/discovery/multiobjective-optimization.html. 